\title{
BIOPHYSICAL CHARACTERIZATION OF THE STRUCTURE AND FLEXIBILITY OF THE E. COLI RIBOSOME
}

\author{
A Thesis presented to \\ the Faculty of the Graduate School \\ at the University of Missouri
}

In Partial Fulfillment

of the Requirements for the Degree

Doctor of Philosophy

by

EMILY DORIS ARMBRUSTER

Dr. Peter V. Cornish, Thesis Supervisor

DECEMBER 2019 
The undersigned, appointed by the Dean of the Graduate School, have examined the dissertation entitled:

\section{BIOPHYSICAL CHARACTERIZATION OF THE STRUCTURE AND FLEXIBILITY OF THE E. COLI RIBOSOME}

presented by Emily Doris Armbruster, a candidate for the degree of Doctor of Philosophy and hereby certify that, in their opinion, it is worthy of acceptance.

Dr. Peter V. Cornish

Dr. Donald H. Burke

Dr. Helmut Kaiser

Dr. Gavin King 


\section{DEDICATION}

For my sisters. 


\section{ACKNOWLEDGMENTS}

Thank you to Dr. Sai Venkatesh (Venky) Pingali, for training me to think as a scientist. You have provided guidance and instruction that took many hours of your time over the past two to three years; this has been most fruitful in molding me as a scientist. Your tenacious approach to time optimization and clarity in communication taught me how to be more efficient in my work. Thank you for teaching me important lessons in preservation. Thank you for comprehending and believing in my ability and my ideas.

Thank you to Dr. Carina Collins and Ashley Campbell for the laughs, the science, the drinks, the meals, and the walks we shared together that have enriched my life. My experience in these mentor-mentee relationships has been more spirited and positive than I could have imagined. Thank you to Dr. Anahita Zare and Dr. Lauren Radford for being my altruistic science lady squad. I have learned a great amount from you and laughed a great amount with each of you.

Thank you to Dr. Haskell Taub and Dr. Helmut Kaiser for navigating me forward. Thank you for demonstrating and sharing your enthusiasm for neutron scattering for it taught me how to endorse a field or technique.

Thank you to Dr. Gerald Hazelbauer and Dr. Lin Randall for relentlessly supporting even the most deviant of graduate students. You both recognize that humans can quickly and subtly change, which allows for the creation of a rapport necessary to produce good science and good scientists. 
Thank you to my comrades at University of Missouri. Thank you to my graduate school class troupe: Dr. Megan Sheridan, Dr. Widler Casy, Dr. Manan Shah, Dr. Erica Lamontagne, Ryan Harvey, and Ben Meredith for sticking together. A special thanks to Dr. Casy for his fervent goodwill and leadership inside and outside of the lab. Thank you to my colleagues at Oak Ridge National Lab. Thank you Dr. Brinda Selvaraj, Dr. Zhi Yang, Dr. Riddhi Shah, Viswanathan Gurumoorthy and all the others on the 2nd floor at SNS that created an exceptional work environment. Thank you to Dr. Kevin Weiss for your help and guidance. A very special thanks to Qiu Zhang for your support and geniality. Thank you to the community of scientists, doctors, and professors I grew to know at Hope College for setting the scaffolding that supported my progress.

Thank you to my thesis supervisor Dr. Peter V. Cornish for accepting me into the lab and providing an environment for me to develop my biochemistry skill set. Thank you to my committee members Dr. Gavin King, Dr. Donald H. Burke, Dr. Helmut Kaiser, and past member Dr. Renee Jiji for the guidance each has provided throughout my graduate school career. 


\section{Contents}

Acknowledgements ii

List of Tables vi vi vis

List of Figures vii

1 Introduction 1

1.1 Bacterial Translation . . . . . . . . . . . . . . . . 1

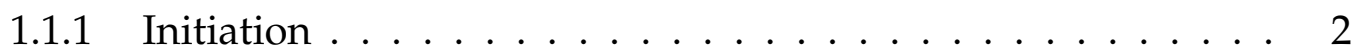

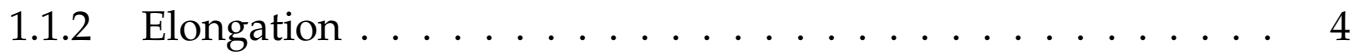

1.1.3 Termination, Release, and Rescue . . . . . . . . . . 5 5

1.2 Extended Roles and Regulation of the Ribosome . . . . . . . . . . . . 6

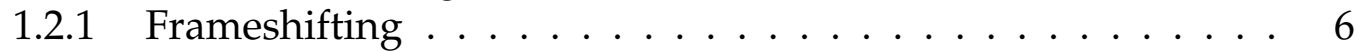

1.2.2 Co-Translational Translocation . . . . . . . . . . . . 8

1.2 .3 Stationary Phase .................... 9

1.3 Small Angle Scattering Experiments of Biological Molecules . . . . . 10

1.3 .1 Scattering Length Density . . . . . . . . . . . . . . . . 12

1.3 .2 Contrast Matching $\ldots \ldots \ldots \ldots$. . . . . . . . . . . . . . . .

1.4 Extracting Structural Data from Small Angle Scattering Experiments 15

1.4.1 Data Reduction and Subtraction . . . . . . . . . . . 16

1.4 .2 Guinier Analysis . . . . . . . . . . . . . . . . . . . 18

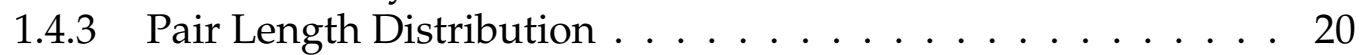

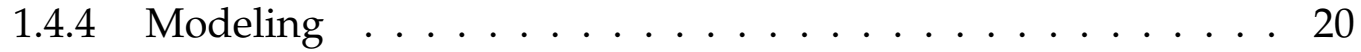

1.5 Capturing the Flexibility of the Ribosome in Solution . . . . . . . . 22

2 Ribosome Purification and Characterization 40

2.1 Ribosome Purification Methods . . . . . . . . . . . . . . . . . . . . . . 40

2.1 .1 Classical Purification . . . . . . . . . . . . . . . 40

2.1 .2 Novel Purification Techniques . . . . . . . . . . . . 42

2.1.3 Application of a Novel Purification Method . . . . . . . . . 42

2.2 Ribosome Characterization Methods . . . . . . . . . . . . . . 43

2.2.1 SDS Polyacrylamide Gel Electrophoresis . . . . . . . . . . . . . 44

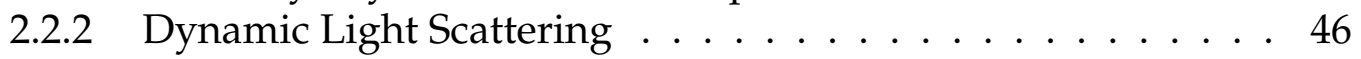

2.2.3 Electron Microscopy . . . . . . . . . . . . . . 50 5

3 Structural Performance of Ribosomal Proteins during mRNA Unwinding by E. coli Ribosomes in Solution

3.1 Summary . . . . . . . . . . . . . . . . . . 6 61

3.2 Introduction $\ldots \ldots \ldots \ldots \ldots \ldots \ldots \ldots$. . . . . . . . . . . . . . . . .

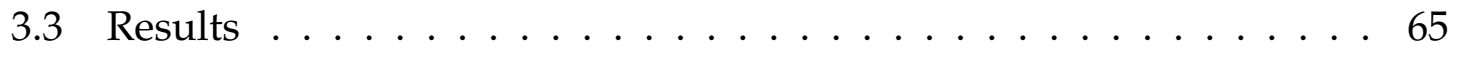

3.3.1 Small-Angle Scattering observes intermolecular dimensions of the rRNA and the ribosomal proteins $\ldots \ldots \ldots$. . . . . 65 
3.3.2 Solution Scattering is from the Flexible Ribosome in Solution 66

3.3.3 Characterizing the Conformations of the E. coli 70S Ribosome

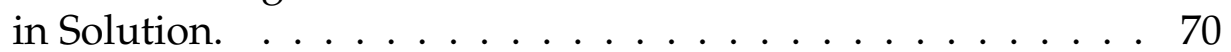

3.4 Discussion . . . . . . . . . . . . . . . . . . . . . 71

3.4.1 Extension of Ribosomal Protein in Ribosomal Helicase Mech-

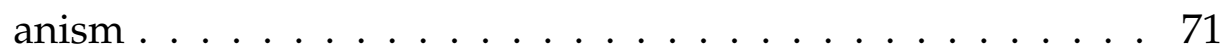

3.4.2 Ribosomal Proteins are Flexible and Interact with Water . . . 72

3.5 Methods $\ldots \ldots \ldots \ldots \ldots \ldots \ldots \ldots \ldots \ldots \ldots$

4 The Influence of mRNA Secondary Structure Stability on Ribosomal Sub\begin{tabular}{|l|l|}
\hline unit Rotation & $\mathbf{8 1}$ \\
\hline
\end{tabular}

4.1 Summary . . . . . . . . . . . . . . . . . . . . . 81

4.2 Introduction $\ldots \ldots \ldots \ldots \ldots \ldots$

4.3 Methods . . . . . . . . . . . . . . . . . . . . . 8 83

4.3.1 Creation of Macromolecular Components . . . . . . . . . . 83

$4.3 .2 \quad$ smFRET Microscopy and Analysis $\ldots \ldots$. . . . . . . . . . 85

4.4 Results and Discussion . . . . . . . . . . . . . . . . . 89

4.4.1 Visualization of Subunit Population During Helicase Activity 89

4.5 Future Work $\ldots \ldots \ldots \ldots \ldots \ldots \ldots \ldots \ldots$

5 Probing the Bacterial Co-Translational Translocation Pathway 99

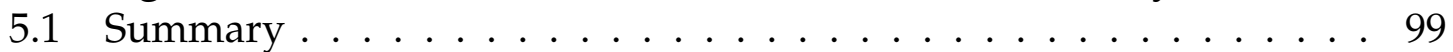

5.2 Introduction $\ldots \ldots \ldots \ldots \ldots \ldots \ldots \ldots$

5.3 Methods . . . . . . . . . . . . . . . . . . . . . 102

5.3.1 In vitro Co-translational Translation Assay Development . . . 102

5.3.2 Optimizing AFM Imaging of Biological Samples in Liquid . . 106

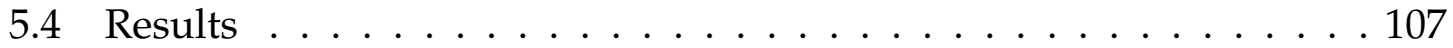

5.4 .1 Ribosome Nascent Chain Characterization . . . . . . . . . 107

5.4.2 The Ribosome on Mica Substrate: As Seen by AFM . . . . . . 108

5.4.3 Ribosome Nascent Chain Non-specifically Binds to Lipid Bi-

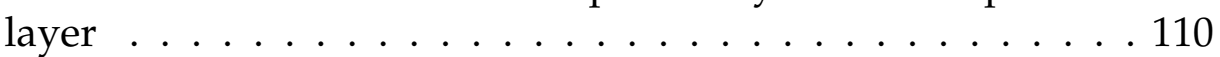

5.5 Discussion and Future Work . . . . . . . . . . . . . . . . . . 112

6 Perspectives in Expanding the Ribosomal Frontier 119

7 Appendix 122

7.1 Supplementary Figures $\ldots \ldots \ldots$. . . . . . . . . . . . . . . . . .

Vita

130 


\section{List of Tables}

$1 \quad$ Guinier analysis and GNOM P(r) fitting . . . . . . . . . . . . 67

$2 \quad$ Primers used to clone SecM. . . . . . . . . . . . . . . . . . . . 104

3 Primers used for point mutations to insert AUG. . . . . . . . . . . . 104

$4 \quad$ Primers used for point mutations to make end leader peptide with alanine and stop codon AUG. . . . . . . . . . . . . . . . . . . . 104

5 Sequence of the serine suppressor tRNA. . . . . . . . . . . 105

$51 \quad$ Weights of 50 S large subunit proteins . . . . . . . . . . . . . . 124

S2 Weights of $30 \mathrm{~S}$ small subunit proteins . . . . . . . . . . . 125

S3 Oligo sequences that were bought from Integrated DNA Technologies.125

S4 $\quad$ Extended Guinier analysis of ribosomes bound to structured mRNA in $100 \% \mathrm{D}_{2} \mathrm{O}$ buffer. . . . . . . . . . . . . . . . . 126

S5 Sequence of cloned m291 DNA. . . . . . . . . . . . . . . . . . 126

S6 Maximum dimension, $\mathrm{D}_{\max }$, calculated from the all atom models. . . 127

S7 Radius of gyration, $R_{g}$, calculated from the all atom models. . . . . . 128

S8 $\quad$ smFRET histogram fitting parameters. . . . . . . . . . . . . . . 129 


\section{List of Figures}

$1 \quad$ Critical steps of translation. $\ldots \ldots \ldots \ldots \ldots \ldots$

$2 \quad$ Three cellular process that utilize the ribosome beyond normal mode cytosolic translation. . . . . . . . . . . . . . . . . .

3 Setup of a Small Angle Scattering (SAS) Experiment. . . . . . . . . . 11

4 Schematic of buffer subtraction. . . . . . . . . . . . . . . 11

5 Example of SAS data before reduction $\ldots \ldots \ldots \ldots$

6 SANS buffer and sample curves before subtraction . . . . . . . . 17

$7 \quad$ SDS-PAGE profiles $\ldots \ldots \ldots \ldots \ldots \ldots \ldots$

8 Ribosome population found using DLS . . . . . . . . . . 48

9 DLS of sucrose gradient fractions. . . . . . . . . . . . . . . . 49

10 Populations monitored via DLS. $\ldots \ldots \ldots \ldots$

11 3D model of the 70 S E. coli ribosome . . . . . . . . . . . . . . . 53

12 Negative Stain 2D classifications . . . . . . . . . . . . . 54

13 Schematic of scattering contributions from protein and rRNA ... . 64

14 Scattering intensities and GNOM P(r) fit (left) $\ldots \ldots \ldots$

15 Schematic of tethering a ribosome complex in an smFRET experiment. 86

16 Example of a trace . . . . . . . . . . . . . . . . . . 88

17 MFE structures of mRNA $\ldots \ldots \ldots \ldots$

18 smFRET histograms $\ldots \ldots \ldots \ldots \ldots \ldots \ldots$

19 Setup of an Atomic Force Microscope with tapping motion visualized.101

20 Product of In vitro Co-Translational Translation reactions . . . . . . . 107

21 Tapping mode AFM images of Ribosomes on mica substrate. . . . . . 108

22 Height density histograms . . . . . . . . . . . . . . . . . . . . 110

23 AFM images of lipids and ribosome nascent chain complexes . . . . 111

51 Profile of the protein standards. . . . . . . . . . . . . . . 122 
S2 $\quad$ Negative Stain model with PDB overlayed . . . . . . . . . . . . 122

S3 $\quad$ SAXS experimental curves overlayed. . . . . . . . . . . . . . . 123

S4 $\quad$ SDS-PAGE analysis of tagged ribosomes . . . . . . . . . . . . . . 123 


\section{Introduction}

\subsection{Bacterial Translation}

At the discovery of ribosomes by George Palade in 1955 in the first image of the subcellular environment, he described them as "a particulate component of small dimensions (100 to $150 \AA$ ) and high density". Subsequently, the ribosome was shown to be the site of protein synthesis, or translation, and thus an essential macromolecular complex for all cells.

Ribosomes can have variability from species to species, but the overall structure and function are conserved [66]. Ribosomes are named according to their sedimentation coefficients, a unit of density expressed in Svedbergs (abbreviated S). The three most studied and most prevalent ribosomes are the bacterial 70S, the eukaryotic $80 \mathrm{~S}$, and the $55 \mathrm{~S}$ mitoribosome, which is present in the mitochondrion organelle. The bacterial ribosome serves as a target for many antibiotics and is a model system for investigating the structure and function of this "nanomachine". Despite variations in size, all ribosomes consist of a small and a large subunit that when bound together have an internal cavity that is divided into three sites, named A, $\mathrm{P}$, or E site. The bacterial ribosome has a $30 \mathrm{~S}$ small subunit, which consists of a $16 \mathrm{~S}$ rRNA and 21 attached proteins, and a 50S large subunit that is made up of the $23 \mathrm{~S}$ rRNA, 5S rRNA and 31 proteins. This dissertation discusses the $70 \mathrm{~S}$ bacterial ribosome, other than when the $80 \mathrm{~S}$ eukaryotic ribosome is specified.

Work has been done to understand this complex machinery and elucidate the conformational motions and molecular forces that orchestrate the synthesis of nascent peptides in a process called translation. Translation is the process of using tRNA, which provide the amino acid monomers, and mRNA, which provides the code of which amino acids to link together, to create a new protein. Translation can be 
broken down into three main phases: Initiation, Elongation, and Termination.

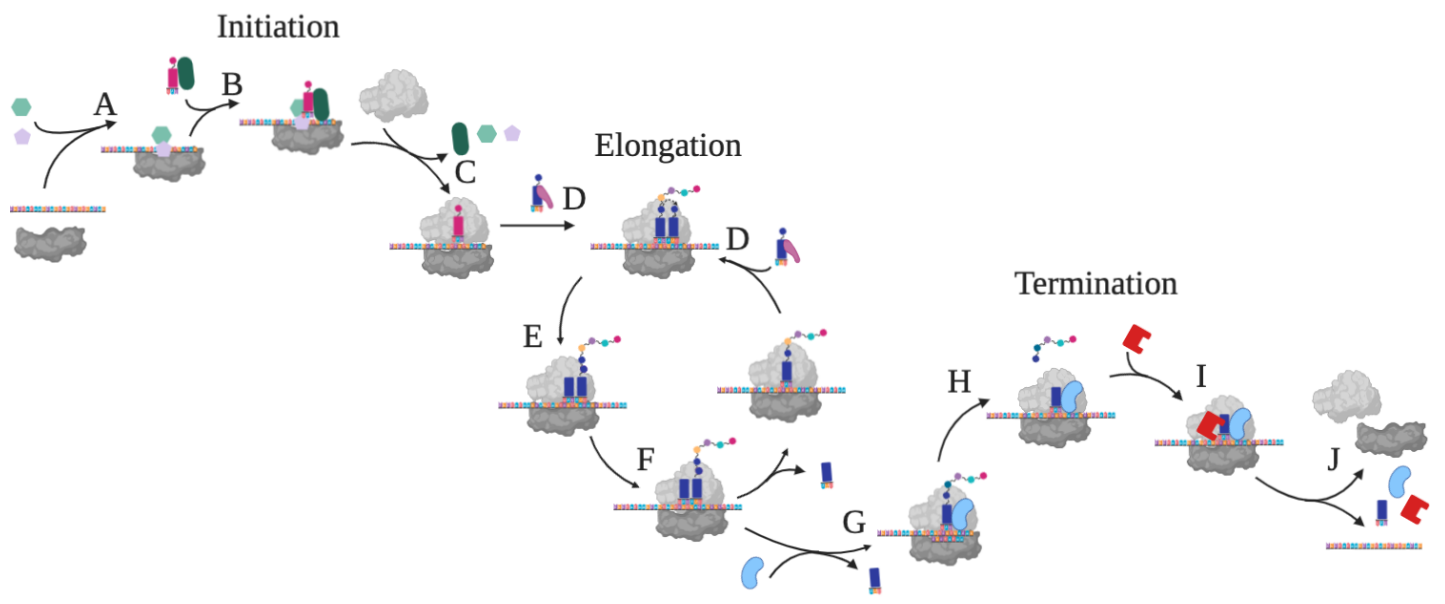

Figure 1: Critical steps of translation.

\subsubsection{Initiation}

The initiation regulates protein synthesis by defining which RNA in the cell gets translated into proteins [69]. As the ribosome reads mRNA, three nucleotides code for one amino acid. Initiation that does not occur properly on the first three nucleotides, or "in frame," will lead to the ribosome misreading the entire nucleotide code.

The initiation process of every ribosome uses tRNA carrying an $\mathrm{N}$-formylmethionine amino acid (fmet-tRNA ${ }^{\text {fmet }}$ ); beyond this commonality, the process of initiation varies from organism to organism. Eukaryotic initiation exhibits far greater complexity compared to the bacterial process. A comparison of eukaryotic and bacterial initiation illustrates the typical complexity differences between the two translational systems.

Eukaryotic initiation has large protein co-factors and proactively regulates mRNA recruitment. Eukaryotic initiation factor 3 (eEIF3) is $800 \mathrm{kDa}$ and consists of 13 subunits [30]. Cellular machinery select which mRNA should be translated by 
adding additional nucleotides to the $5^{\prime}$ end of mRNA [61]. The mechanisms of eukaryotic initiation force viruses to take measures to ensure their foreign RNA gets translated. For example, some viruses produce large RNA motifs, named Internal Ribosome Entry Sites (IRESs), that instigate translation of uncapped mRNA in eukaryotic host cells[49]. In general, the bacterial system initiates with fewer co-factors.

Initiation of the $70 \mathrm{~S}$ complex involves the assembly of tRNA, mRNA, the $30 \mathrm{~S}$ small subunit, and three small initiation factor proteins (IF1, IF2, IF3). The most common mode of initiation is the "30S binding mode" initiation path. This path creates the $30 \mathrm{~S}$ preinitiation complex (pre-IC). Firstly, IF3 and IF1 bind to a free $30 \mathrm{~S}$ subunit, where IF3 can sterically hinder the $50 \mathrm{~S}$ subunit from premature association [53](A, Figure 1). 30S subunit binds to the Shine Dalgarno sequence and AUG start codon in the mRNA [71]. Binding of the mRNA is thought to occur at any time during the formation of the $30 \mathrm{~S}$ pre-IC. Prokaryotic mRNA has defined translation initiation region (TIR). The TIR includes the Shine Delgarno sequence, which situates the ribosome correctly on the mRNA. Variations within the TIR nucleotide code has an impact on the efficiency in which individual transcripts are translated [25]. fmet-tRNA ${ }^{\text {fmet }}$ binds to the P-site and is maintained in the P-site by IF2 and IF1 while IF1 also sterically prevents any tRNA from binding in the Asite (B, Figure 1). After these components are assembled, the 30S pre-IC undergoes a conformational change to create the $30 \mathrm{~S}$ Initiation Complex (IC). The 30S IC is able to bind a $50 \mathrm{~S}$ subunit, and the initiation factors dissociate to create the $70 \mathrm{~S}$ ribosomal complex (C, Figure 1). 


\subsubsection{Elongation}

After a 70S ribosome has become compatible for elongation, the A-site is accessible for new tRNA binding. Delivery involves a complex made of aminoacylated tRNA, EF-Tu, and GTP (D, Figure 1). This delivery complex will bind the tRNA anticodon to the mRNA codon exposed in the A-site (E, Figure 1). Before the tRNA is released from the delivery complex, the complementary anticodon:codon interactions are audited by the ribosome. If the anticodon:codon interactions are correct a bend in the tRNA occurs and GTP hydrolysis occurs in the complex [67, 46]. EF-Tu is released, and the aminoacyl tRNA undergoes conformational rearrangements to align peptidyl site to Peptidyl Transferase Center (PTC).

Both $\mathrm{C}$ terminal ends of the P-site tRNA and A-site tRNA are situated in the PTC, the former with a peptide chain attached and the latter with an aminoacyl moiety attached. Bond formation is initiated by the alpha-amino group from the A-site tRNA aminoacyl moiety attacking the carbonyl carbon of the peptidyl-tRNA [28]. The reaction is enhanced by the 2'-OH residue of the P-site tRNA along with water coordinated hydrogen bonding by A-2451 [84, 44]. After the peptide bond is formed, the P-site contains a deacylated tRNA and the A-site contains a peptidyl tRNA (E, Figure 1). As the process continues, and the nascent peptide grows longer. The newly formed peptide sits in the ribosome exit tunnel that is connected to the PCT and eventually exits the ribosome [57]

tRNAs must be moved to their adjacent sites in the ribosome to ultimately create a vacant A-site for a new tRNA to bind (F, Figure 1). This is necessary in order to continue elongation as it will allow for a new aminoacyl-tRNA to bind to the Asite. tRNA molecules are moved through the ribosome in a method called translocation. During translocation, the tRNA must be moved simultaneously with the mRNA and retain a binding junction that is made up of 3 nucleotides per each 
molecule, an anticodon:codon pairing of the tRNA:mRNA, respectively, in order to keep the proper reading frame for nascent peptide. Translocation can occur spontaneously but when aided by a GTPase named Elongation Factor G, translocation is about 15,000 more efficient [54]. Understanding the dynamics and the structural changes that occur during translocation have been investigated in the field of ribosome research. The understanding of these structural changes are novel glimpses into the functionality of a large, multicomponent macromolecules. complex [10, 9].

\subsubsection{Termination, Release, and Rescue}

Elongation must come to an end to conclude the process of a proteins production, as the nascent peptide must be released from the tRNA and ejected to the environment outside of the exit tunnel. Standard peptide release starts when a stop codon reaches the A-site of the ribosome. A stop codon is not bound by another tRNA molecule, but rather two proteins have the ability to bind to a stop codon, these proteins are Release Factor 1 and 2 (RF-1, RF-2) (G, Figure1). After one of these release factors bind, they stimulate a hydrolysis reaction which releases the nascent peptide from the P-site tRNA [45] and then Release Factor 3 (RF-3) binds and assists in the release of RF-1 and RF-2 from the ribosome. Structural studies suggest that RF-3 binding induces a hybrid state that induces the release of RF-1 and RF-2 [38](I, Figure 1). Ribosome Recycling Factor plays a role that prevents the binding of free tRNAs in the A and P-sites and stimulates breaking subunit bridges, thus aiding in the dissociation of the ribosome [15]. After ribosome dissociation, the 50S and $30 \mathrm{~S}$ subunits are free for initiation of the next protein to synthesize (J, Figure 1).

Approximately $5 \%-10 \%$ of ribosomes come upon the end of an mRNA strand without reading a stop codon in the mRNA [40, 34]. These ribosomes will not 
dissociate independently and need to be rescued. If the ribosomes are not rescued a significant amount of ribosomes would be stuck at the end of an mRNA strand and unable to translate new proteins; this situation can be lethal [70, 13]. Three main molecules in charge of rescuing these ribosomes are transfer-messenger RNA (tmRNA) or a complex made of Alternate Release Factor A (Arf A) and RF-2. AfrA senses the vacant mRNA tunnel and activates RF-2 to mediate peptide release [13. 70]. tmRNA can also be used for rescue. tmRNA, carrying an aminoacyl moiety of alanine, is accommodated into the A-site by $\mathrm{SmpB}$ and both have to be translocated to the P-site by EF-Tu, where a reaction with the growing peptide chain occurs [52].

\subsection{Extended Roles and Regulation of the Ribosome}

Many cellular processes extend ribosomal function beyond the basic translation process of initiation, elongation, and termination as it is described above. Below are three extensions of normal mode translation that show the ribosome can be dictated, directed, and deactivated (Figure 2). The ribosome is susceptible and controlled by imparting on small forces on the complex, specific examples detailed below elucidate forces that are on the order of picoNewtons and the strength of one hydrogen bond [33, 79]. These extensions of normal mode translation elucidate the dynamic and flexible nature of the ribosomes structure.

\subsubsection{Frameshifting}

As peptide elongation proceeds, the ribosome moves along the mRNA strand. In solution, a single-stranded RNA, such as an mRNA molecule, can fold into secondary structures such as hairpins and pseudoknots [22]. As the ribosome moves along an mRNA stand it can come upon double-stranded and triple-stranded structures in the mRNA (Left, Figure 2). The tunnel for mRNA in the ribosome is only 


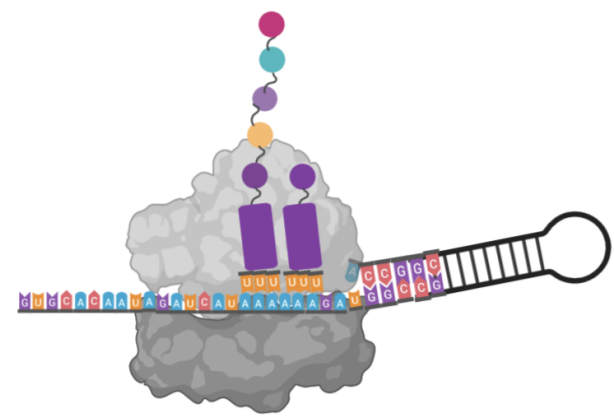

Frameshifting

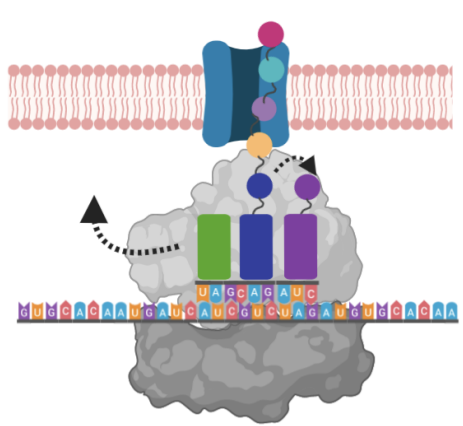

Co-Translational

Translocation

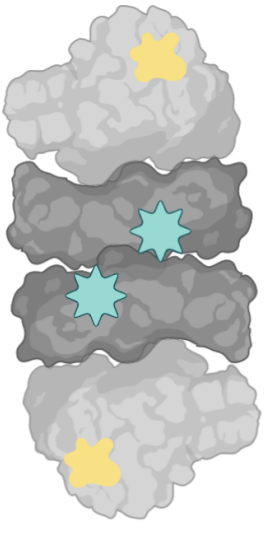

Stationary Phase

Figure 2: Three cellular process that utilize the ribosome beyond normal mode cytosolic translation.

big enough for single-stranded mRNA [13, 16, 20, 39, 41, 48, 50, 87, 92]. In a bacterial system, the ribosome is in charge of unwinding these secondary structures. Interaction with an mRNA secondary structure can stall or slow the ribosome and can induce a slippage of the mRNA:tRNA junction, causing a change in the reading frame [7, 32]. If the reading frame shifts by +3 nucleotides, the number of nucleotides that make up a codon, one amino acid is omitted, but the following mRNA code is conserved. More commonly, the frame shifts by +1 or +2 nucleotides, and the entire code carried in the mRNA is rewritten to a new set of codons. Current literature suggests that a frameshifting event often results in a change of the protein being produced or truncation of a protein [2]. Frameshifting can happen in a reliable manner; for example, $a+1$ frameshift is prompted by DnaX $80 \%$ of the time in vivo and produces $4 \gamma$ subunits for each $\tau$ subunit in DNA Polymerase III [8,79, 89]. Structured mRNA that has a defined frameshifting efficiency is called programmed frameshifting [65]. Programmed frameshifting allows the genome to contain more information that what is linearly encoded, as one mRNA can be translated to produce more than one protein [35, 83]. This structural programming phenomenon also provides regulation of the proteome that is not 
reliant on protein co-factors, as an mRNA can frameshift and induce a truncated, non-functional protein [2]. Frameshifting is sensitive to the forces on the scale of two hydrogen bonds [79] which indicates the ribosome's sensitivity to manipulative forces. The flexibility of the ribosome and sensitivity of this mechanism make frameshifting a difficult phenomena to study using structural techniques that provide forces that could push the delicate structural equilibrium, such as x-ray crystallography and Cryo-EM. In Chapter 3 and 4 this mechanism is studied using techniques that investigate the frameshifting ribosome in solution at ambient temperatures.

\subsubsection{Co-Translational Translocation}

In contrast to the process of mRNA dictating frameshifting, the ribosome can be directed by the emerging nascent peptide chain stalling or recruiting cofactors during the production of inner membrane proteins (IMPs) [77]. Most IMPs on the plasma membrane are manufactured by a ribosome that is docked atop a protein channel called a translocon (Middle, Figure 2). Multiple translocons have been identified in both bacterial and eukaryotic systems, though it largely remains undetermined which IMPs are made with which translocon [55, 85]. The process of creating an IMP is called Co-Translational Translation. This process shows the ribosome in a supporting role, as a component part of a larger system [5, 43, 42, 47, 85].

Previous studies indicate that stalling of translation, transit to the membrane, and binding to the translocon are all regulated by the nascent peptide emerging from the ribosome's exit tunnel [23, 58, 77, 91]. The forces that allow the nascent peptide chain to dictate the translating ribosome are small, on the order of picoNewtons [33]. The macromolecules involved in the co-translational transloca- 
tion pathway rely on the binding kinetics of their individual parts, which allows for many sub-populations and intermediates that are difficult to detect in bulk biochemistry experiments [47, 88, 90]. Thus, single-molecule experiments are necessary to resolve the structure, function, and dynamics of these complex systems and correlate them to the product they create. A study using single-molecule experiments and accompanying bulk assays is outlined in Chapter 5 .

\subsubsection{Stationary Phase}

As the importance of a macromolecule increases, so does the necessity to regulate it. One mechanism of regulating ribosomal function is deactivation. Ribosomes are heavily utilized while bacteria are growing to produce protein for expanding and

dividing cells. As the environment surrounding bacteria changes, such as a drop in nutrients or other factors that cause stress on a bacterial cell, the cells can enter a stationary phase of growth. During stationary phase, energy is conserved and the cellular mechanisms of expansion shut down. Bacteria can slow down protein synthesis by converting 70S ribosomes from an active state to an inactive state [1] or to $100 \mathrm{~S}$ dimers [82]. When the stressor disappears or nutrients increases, the cell most fit and ready to efficiently utilize the opportunity will grow. Thus, it is better to inactivate, rather than degrade, the $70 \mathrm{~S}$ ribosome, because the ribosome costs a cell time and energy to produce [56].

Bacteria use specific proteins to sequester the active $70 \mathrm{~S}$ complexes. The Ribosome Modulation Factor (RMF) and Hybernation Promoting Factor (HPF) dimerizes two $70 S$ ribosomes to form $100 S$ complex. RMF has been shown to dimerize the two $70 S$ to create a premature $90 \mathrm{~S}$ complex, and the HPF is a necessary addition to create the mature $100 \mathrm{~S}$ complex [81]. Any bacteria can usually have at least one homolog of HPF, which can be influential in the creation of the 100S complex 
[81]. for example, the HPF homolog YfiA will prevent the 70S dimerization [80]. In the bacterial 70S the RMF binds to the 50S and the HPF binds to the 30S during 100S dimerization (Right, Figure 2] [3]. In this way, functions of the ribosome functions are halted without disassembling the ribosome. It is important to manage the ribosome because it is entrenched in creating the proteome and phenotype of a cell.

\subsection{Small Angle Scattering Experiments of Biological Molecules}

The lack of sample manipulations make Small Angle Scattering ideal to study the ribosome during its delicate role in frameshifting. The size and shape of a ribosome that is untagged, unfrozen, and tumbling in solution, can be found using SAS if the ribosomes are dilute and monodispersed. The SAS instrument setup has three major components: the incident radiation, the sample, and the detector (Figure 3). When an incident beam hits a sample, it can either pass through, be absorbed, or be scattered by the sample. The spatial distribution of the scattering intensities captured by the detector informs on the structure of the scattering macromolecule. Analysis of small angle scattering experiments is based on four important assumptions. The necessary experimental assumptions are that the distance between the detector and scattering sample is large, on the order of meters, and that there is insignificant energy transfer or absorption. Assumptions about the beam are that the sample does not distort the incident beam and that an incident wave is regarded as a plane wave and the scattered a spherical wave. If all these assumptions are satisfied, SAS measurements can be analyzed in the methods outlined below.

The information captured by a measurement is based on the type of incident beam used; this dissertation addresses the use of neutrons and x-rays. These beams 


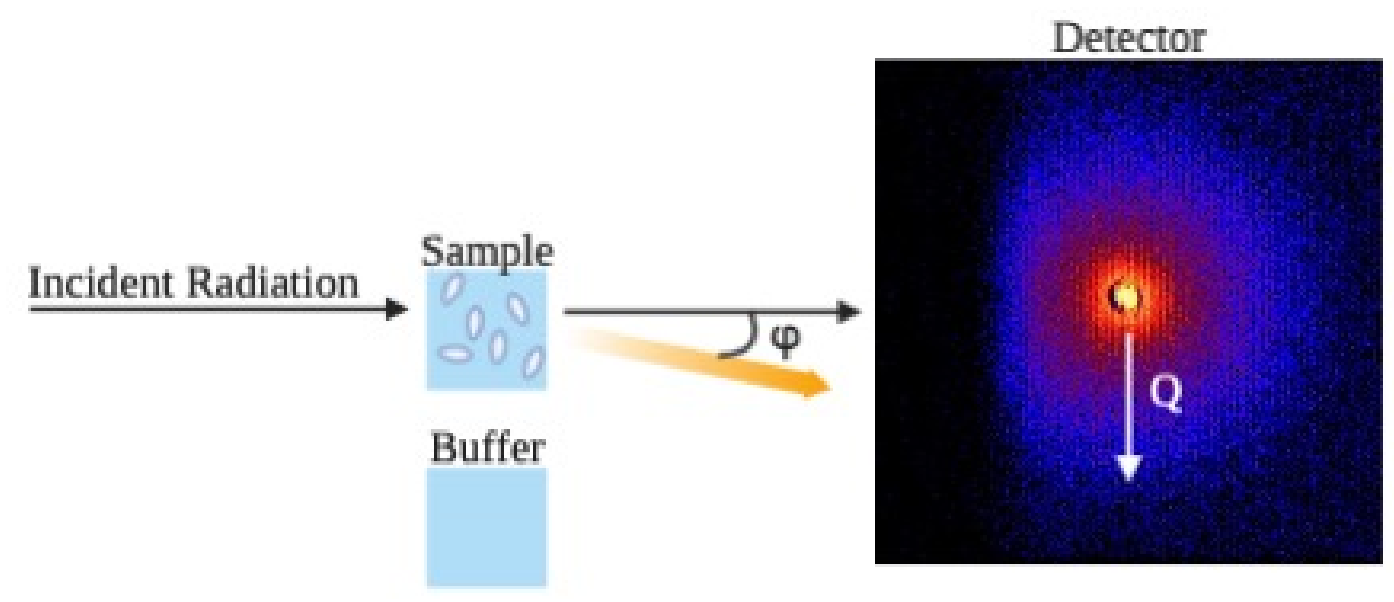

Figure 3: Setup of a Small Angle Scattering (SAS) Experiment.

can scatter in four different modes, inelastic and elastic scattering, which both have coherent and incoherent subcomponents. Elastic scattering conserves the wavelength $(\lambda)$ and energy between the incident and scattered beams, unlike inelastic scattering that includes a momentum exchange. Coherent scattering means that the scattering happens in a nonrandom and forward direction compared to random phase scattering called incoherent scattering. Structural information comes exclusively from coherent elastic scattering, though both incoherent elastic and coherent elastic scattering are captured on a SAS detector.

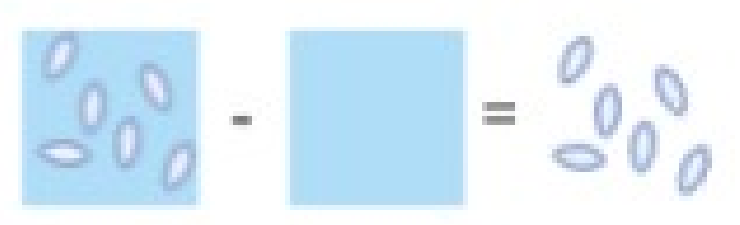

Figure 4: Schematic of buffer subtraction.

The scattering pattern, $I_{\text {total }}(q)$, is the resulting interference of all the scattered waves [27]. Dilute monodispersed solutions scatter in an isotropic manner, equally in every direction, dependent only on $q$ (Figure 3 ). The scattering at each value of $q$ can be understood in Equation 1 where $\lambda$ is wavelength of radiation, and $2 \phi$ is 
the scattering angle of a scattered wave.

$$
q=\frac{(4 \pi \sin \phi)}{\lambda}
$$

In SAS the molecules in the sample can have two scattering contributions, intramolecular scattering and inter-molecular scattering, which are $I(q)$ and $S(q)$ and called the form factor and structure factor, respectively, as seen in Equation 2. When there is no intermolecular ordering, such as when macromolecules are independently tumbling around in solution and not interacting with one another, the only scattering contribution to the signal will be the form factor $I(q)$.

$$
I_{\text {total }}(q)=I(q)+S(q)
$$

\subsubsection{Scattering Length Density}

Experiments require separate measurements of pure buffer and the macromolecule in buffer, referred to as the sample. The scattering from the macromolecule is found by subtracting the buffer measurement from the sample measurement. When buffer is a featureless matrix of water and salt, the scattering signal, $p s$, is solely incoherent scattering. The difference in the scattering lengths of the macromolecular particle and the equivalent solvent volume is the excess solvent density, $p r$, as seen in Equation 3 . Scattering of macromolecular assemblies can be understood by the pair length distribution $p(r)$, which is addressed later in this chapter.

$$
\Delta p r=p(r)-p s
$$

The likelihood of an atom to scatter the incident beam is defined by the atom's scattering length, a parameter correlated to the incident beam energy. Neutrons 
and X-rays scatter with different interactions: a neutron scatters off the nucleus of an atom whereas an x-ray photon scatters off of the electron cloud. The probability of $\mathrm{x}$-ray scattering increases as the atomic number increases because the scattering length for $\mathrm{x}$-rays is related to the electron density. As macromolecules are made up of more than one atom, the scattering length of all atoms are summed to determine the scattering length density (SLD) of the entire molecule. A molecules SLD for xrays is defined by Equation 4 where $\mathrm{Z}$ is the atomic number of the $i$ th atom in a molecule with a molecule volume of $V_{m}$ and $r_{e}$ is the classical radius of the electron which is $2.81 \times 10^{-13} \mathrm{~cm}[31]$.

$$
S L D=\frac{\sum_{i=1}^{n} Z r_{e}}{V_{m}}
$$

A neutron scatters after an interaction with an atoms nucleus. This scattering lengths differs from $x$-ray scattering length because it does not follow a periodic trend and is isotope sensitive. As each isotope has a distinct scattering length, the neutron SLD of a macromolecule is defined in Equation 5 where $b_{c}$ is the coherent scattering length of the $i$ th of $n$ atoms in a molecule with a molecule volume of $V_{m}$ [37, 68, 51].

$$
S L D=\frac{\sum_{i=1}^{n} b_{c}}{V_{m}}
$$

The SLD of a molecule is dependent on the density of atoms in space, which is defined by volume, $V_{m}$. Similarities in the atomic composition of biological molecules mean that any protein will have a similar SLD to any other protein and any RNA will have a similar SLD to any other RNA. For biological molecules, the x-ray SLD in fixed as a molecule's elemental composition would have to change in order to change the SLD, whereas a biological molecules neutron SLD can be manipulated while conserving elemental composition, using isotopes [51, 68]. Lighter elements 
have a greater contribution to a neutron SLD compared to x-ray SLD, making neutrons an optimal SAS probe to study biological materials. Beneficially, Hydrogen and Deuterium have neutron scattering lengths of -3.741 femtometers (fm) and $6.671 \mathrm{fm}$, respectively, with a negative scattering length indicates a $180^{\circ}$ phase change of the scattered beam. Deuteration, the use of deuterium in place of hydrogen, easily changes the SLD of a macromolecular complex because hydrogen is the most ubiquitous atom in biological molecules. The experimental signal is dependent on the size, concentration, and SLD contrast of the molecule. As the SLD contrast between the macromolecule and the buffer increases, the observed scattering from the molecule is increased (Figure 4). When finding the solution structure of a protein, SLDs can be used to provide contrast and increase signal when the size of a protein is unchangeable, and the protein may aggregate at high concentrations. Deuterium can be used to change the SLD of the protein or RNA molecule by incorporating deuterium into the non-exchangeable hydrogen sites of a molecule.

\subsubsection{Contrast Matching}

Increasing and decreasing contrast between components in the sample can be made by adding deuterium to the buffer. Protein and RNA have a natural SLDs, which can be matched with a combination of $\mathrm{H}_{2} \mathrm{O}$ and $\mathrm{D}_{2} \mathrm{O}$. For example, lipids are contrast matched at $14 \% \mathrm{D}_{2} \mathrm{O}$, RNA at $43 \% \mathrm{D}_{2} \mathrm{O}$, and proteins at $63 \% \mathrm{D}_{2} \mathrm{O}$. When deuterium is incorporated into non-exchangeable hydrogen sites of a protein or RNA, it increases the percentage of $\mathrm{D}_{2} \mathrm{O}$ necessary to reach a contrast match [51]. Increasing a contrast match point to $90 \%$ or even $100 \% \mathrm{D}_{2} \mathrm{O}$ buffer will decrease the number of hydrogens in the sample, which is good because hydrogen has a large incoherent scattering length, specifically 25.274 barns [68]. Decreasing the 
amount of hydrogen in a sample will decrease the amount of noise from incoherent scattering in the signal. Overall the use of deuterium in SANS samples can match out a component, such as RNA or protein, and can be used to optimize experimental signal. The method of extracting structural data of a macromolecular complex from SAS measurements is detailed below.

\subsection{Extracting Structural Data from Small Angle Scattering Experi- ments}

SAS measures scattering from a three-dimensional molecule, captures a two-dimensional image, and is analyzed as a one-dimensional plot (Figure 3). SAS data analysis requires expert training due to the difficulty of extracting a three-dimensional structure from one-dimensional information. [19, 36, 37, 62, 63, 76]. SAS provides an ensemble average of all possible orientations; thus, the relative locations of the atoms are lost and only molecular distances, unassigned to a matrix position or atom type, remain. These distances can be used to find the size and shape of the scattering particle [37] in the same way that a deconstructed cabin could be theoretically reconstructed when given the logs used for the initial build. With a known parameter that the cabin cannot be longer than the longest log, multiple different structures can be hypothesized. Additionally, the area of the base of the cabin is known; applying this parameter decreases the possible structures that could be built with the logs. In this same method, the intra-atomic distances found by SAS can be reconstructed to find the possible shape and size of the molecule, and prior or outside knowledge can be applied during analysis. Due to these limitations, the SAS technique itself can only define structural information to a resolution of 0.6 to $1 \mathrm{~nm}[78]$. 


\subsubsection{Data Reduction and Subtraction}
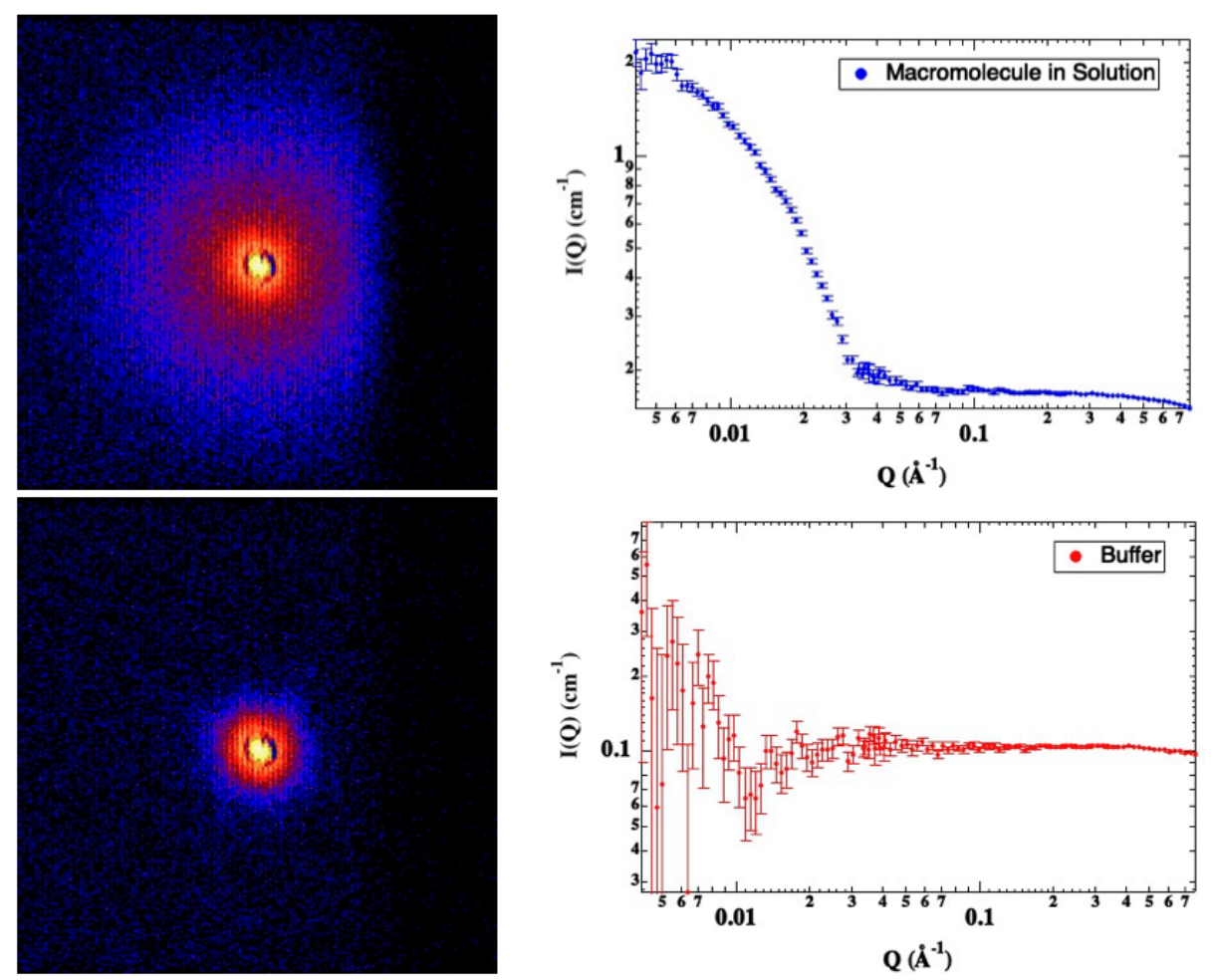

Figure 5: Data before reduction (Left, 2D scattering detected) and after reduction (Right, 1D scattering curve). Top is information of ribosomes in $100 \% \mathrm{D}_{2} \mathrm{O}$ buffer. Bottom is $100 \% \mathrm{D}_{2} \mathrm{O}$ buffer.

The process of converting data from two-dimensional isotropic scattering to a one-dimensional curve is named data reduction (Figure 5). The data reduction process properly situates detected scattering in Fourier space, or $\mathrm{Q}\left(\AA^{-1}\right)$. Precise measurements of the instrument setup and a calibration measurement of material, known as a universal standard, are used in data reduction. The distance between the sample and the detector is needed (Figure 3). Universal standards have distinct scattering patterns that are used to calibrate detected images; standards are usually Porasil B3 for neutrons or silver behenate for x-rays.

Along with plotting the detected scattering in $\mathrm{Q}$, data reduction is a step where the detected scattering is normalized to the amount of beam transmitted by the 
sample. Transmission will vary with sample content and will impact total scattering intensity, a parameter used during analysis. Data reduction can also include other processes such as optimizing the binning of the pixels from the detector or merging scattering collected over multiple detectors. Ultimately, reduction converts a two-dimensional image captured on a detector and outputs a onedimensional scattering curve that is correctly plotted in both X ( $\mathrm{Q}$ in $\AA^{-1}$ or $\mathrm{S}$ in $\mathrm{nm}^{-1}$ ) and $\mathrm{Y}\left(\mathrm{I}(\mathrm{Q})\right.$ in $\left.\mathrm{cm}^{-1}\right)$. Subtracting the pure buffer curve from the sample curve is necessary to extract structural information (Figure 4). The subtraction is dependent on the $\mathrm{Q}$ values where incoherent scattering takes over the coherent signal. This process eliminates incoherent scattering contributions and buffer scattering contributions from the data. The resulting curve contains coherent scattering, or structural information, from the macromolecule. The buffer scattering

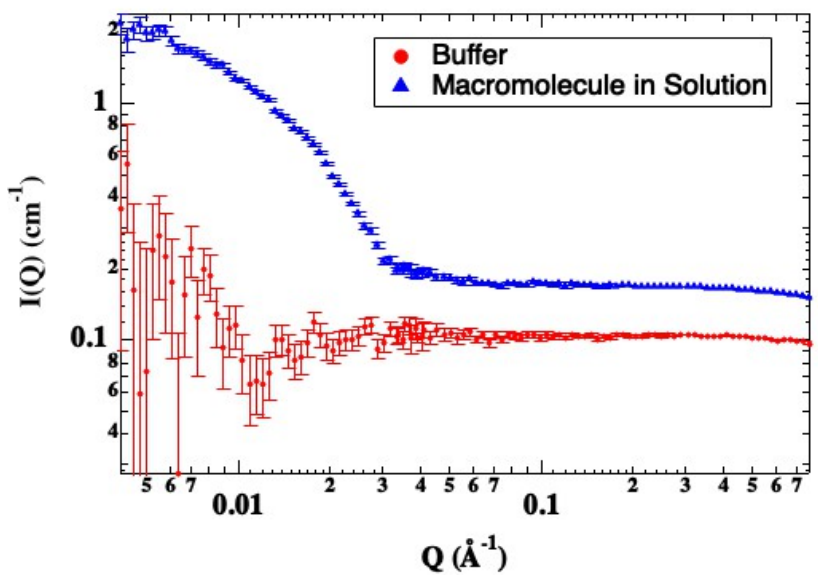

Figure 6: SANS buffer and sample curves before subtraction. The red curve is $100 \% \mathrm{D}_{2} \mathrm{O}$ buffer. The blue curve is ribosomes in $100 \% \mathrm{D}_{2} \mathrm{O}$ buffer.

is flat because the contents are coherent scattering contributions (Figure 6). Incoherent scattering dominates the signal as both curves flatten out to have the same shape at high Q. In SANS the amount of incoherent scattering can be different in the sample and the buffer curves. For example, the blue sample curve reaches the incoherent background at a higher intensity, $I(Q)$, that the red buffer curve, due 
to the incoherent scattering contribution from the hydrogens in the hydrogenated ribosome (Figure 6). Thus, a buffer curve must be multiplied by a scaling factor in order to negate both incoherent and buffer scattering properly. The scaling factor applied to the buffer can impact on the scattering curve that is analyzed, and an expert eye or systematic investigation may be necessary to choose a proper scale factor. Thus, during the analysis methods detailed below, the scale factor used in subtraction must be carefully documented and regarded. After proper reduction and subtraction, the scattering curve can be analyzed to extract the size and shape of the scattering particle.

\subsubsection{Guinier Analysis}

When extracting the structure of a macromolecular complex in solution from a scattering curve, it is critical to understand the dispersity of particles in solution. Polydispersity is a measure of the uniformity of the particles. In order for the scattering to accurately portray a molecules structure, the scattering must be coming from a monodispersed population. A polydisperse population yields ambiguous structural data that can not be sorted. A Guinier analysis is a necessary analysis to understand the dispersity of a particle in a solution.

Due to the data being in reciprocal, as opposed to real space, as $Q$ decreases the atomic length-scale represented at that $\mathrm{Q}$ increases. Thus, the lowest $\mathrm{Q}$ measured contains information about the largest intra-atomic distances observed by the measurement. Capture of the largest dimensions of a monodispersed particle will display a flat region in the low-Q region. Along with the initial appraisal of low $Q$ by eye, a variable-independent analysis, named a Guinier Analysis, must be done to quantify the largest dimensions of the particle.

The range of $Q$ used in a Guinier analysis is defined during the analysis. At 
sufficiently low $Q$, the curve is directly related to the Radius of Gyration $\left(R_{g}\right)$ of the molecule (Equation 6 [26]. The data is plotted as $\ln [I(O)]$ vs $Q^{2}$ and at the slope of this plot can be used to find $\frac{R_{g}^{2}}{3}$ (Equation 7, [26, 27].

$$
\begin{gathered}
I(Q)=I_{o} \exp \left(\frac{Q^{2} R_{g}^{2}}{3}\right) \\
\ln (I(Q))=\ln \left[I_{o}\right] \frac{Q^{2} R_{g}^{2}}{3}
\end{gathered}
$$

While fitting the line to find the slope, constraints on the fit must be considered to confirm the analysis is properly capturing the particle dimensions. For globular particles, such as macromolecular complexes, the $R_{g} \cdot Q_{\max }$ should be between 1.2 and 1.3 [62] with $R_{g}$ being the output from the fit and $Q_{\max }$ being the maximum $Q$ value used in the fit. The amount of data used in the fitting of a logarithmic curve is 1/e which means $Q_{\max } / Q_{\min }$ must be greater than 2 to be a reliable analysis. Expanding the range of $Q$ should be done to observe changes in the Guinier analysis output as the $\mathrm{Q}$ range is changed. A monodispersed particle should not substantially increase or decrease in $R_{g}$ as the $Q$ range expands. In addition to finding the $R_{g}$ in an analysis independent of input variables, the Guinier analysis can be extrapolated to find $I(0)$ which is experimentally inaccessible due to the transmitted beam flooding signal at a $0^{\circ}$ angle. $I(0)$ represents total scattering of a particle and is a function of sample concentration. The value can relate information about the scattering volume and contrast which in turn can be used to find the molecular weight of the scattering particle [36]. Although the Guinier fit is necessary, it is not sufficient to obtain indisputable evidence of the scattering particle's $R_{g}$ or that scattering is from a monodispersed population. 


\subsubsection{Pair Length Distribution}

Along with the Radius of Gyration, the largest particle distance can be found using a variable-dependent Pair Length Distribution function. This method converts the information in the measured scattering curve from reciprocal space $\left(\AA^{-1}\right)$ into real space $(\AA)$ using a Fourier transform $[36,37,62,76]$. The transform output is a $P(r)$-distribution (Equation 3 ) and is essentially a histogram of all the intra-atomic distances of a macromolecule, which is information found in the $I(q)$ scattering curve. $P(r)$ represents the probability of finding a neighboring particle at distance $r$ from another particle [72]. The probability of an $r$ distance drops to 0 once $r$ exceeds the maximum dimension of the particle. Care must be taken to capture the largest dimension of the particle without extending or clipping the dimensions of the particle. In order to correctly calculate the Fourier transform that converts $I(q)$ to $P(r)$, the largest dimension of the particle has to be assessed and selected such that $P(r)$ approaches 0 gradually [78]. Calculating the pair length distribution function provides a second way to measure $R_{g}$ and also defines the largest dimension, or the diameter, of the scattering particle, known as the $D_{\max }$. This analysis yields $R_{g}$ results that are more reliable than a Guinier analysis due to the use of data from the entire curve in the fit [78]. The maximum dimension and $R_{g}$ should be compared to the Guinier analysis and can be compared to dimensions of the molecule taken from literature in order to comprehensively understand and characterize the particle. $D_{\max }$ is necessary for modeling the shape of the scattering particle, which is discussed in the section below.

\subsubsection{Modeling}

Modeling methods have been developed to approach the challenge of determining the three-dimensional structure of a particle from a one-dimensional scattering 
curve. Methods can model data with or without the input of prior knowledge. $A b$ initio modeling programs DAMMIN and DAMMIF use the $P(r)$-distribution function from a single scattering curve as input and uses coarse-grained beads to find the shape of a particle that could give rise to the input $P(r)$ function. A set number of beads are arranged within a volume defined by the $D_{\max }$ of the particle $[18$, 73]. This same technique can be applied using multiple scattering curves from both SANS and SAXS experiments to define a multicomponent particle using the program MONSA [73]. Recently, a software named DENsity from Solution Scattering (DENSS) has been created. DENSS calculates the electron density cloud of a particle from a SAXS scattering curve [24]. The ab initio modeling can be widely applied to model both known and unknown structures. Modeling reproducibility can be tested by applying any appropriate ab initio methods to both SAXS and SANS experimental scattering. These two reasons make ab initio modeling a robust and advantageous technique.

Prior knowledge of a molecule or system can be applied during modeling to understand the complexities of a scattering system beyond the size and shape. As the prior knowledge of a system or molecule can come in all types of reports and characteristics, ranging from Cryo-EM electron densities to binding kinetics, each must be appropriately incorporated during model-dependent data analysis. Prior knowledge is often introduced as constraints in theoretical calculations of scattering. Comparing theoretically calculated scattering to experimental scattering is a common method of analysis in SAS and other types of scattering experiments.

Atomic coordinates, either partially of fully solved by Cryo-EM or X-ray crystallography, can be used during SAS analysis. Multiple programs have been developed to calculate theoretical scattering curves from PDB files [74, 75] and Cryo-EM densities [29, 11]. Cryo-EM and X-ray crystallography both "freeze" the molecule 
of interest in order to capture atomic coordinates, thus the corroborative information provided by SAS measurements can visualize the flexibility of an unfrozen molecule. Flexible and intrinsically disordered proteins are often investigated in a synergistic manner using molecular dynamics [4] or Monte Carlo sampling [11] to model the flexibility of a biological molecule in solution and then compare theoretical curves from these models to the experimental curve. The synergistic use of all atom modeling and MD combined with SAS measurements has grown into a robust and promising method of understanding the structure and function of flexible macromolecules [59].

\subsection{Capturing the Flexibility of the Ribosome in Solution}

The field of functionally oriented structural biology emphasizes the importance of mapping conformational space [17]. Recent advances of single photon detectors allow for Cryo-EM to gain high resolution of a spectrum of molecular conformations in a single sample [6, 14, 21]. These methods have successfully been applied to mapping continuous conformations of the ribosome [12]. SmFRET of ribosomes in solution monitor the structure and flexibility of the ribosome and show there are low energy barriers between the conformations related to protein translation [9. 10]. These smFRET measurements only report on one or two distance measurements in the sample. Assigning the distance changes observed by smFRET to structural dynamics and flexibility requires guidance from global structural data obtained with Cryo-EM or x-ray crystallography [64]. Thus, both techniques rely on one another; smFRET relies on the global structural data from Cryo-EM or x-ray crystallography and Cryo-EM or x-ray crystallography rely on smFRET to understand the relationship and dynamics between solved conformational states. This same relationship occurs with other single-molecule techniques, such as optical 
tweezers, that observe a single parameter and apply the parameter to global structural data in order to understand how the parameter influences ribosome structure and function $[60,89,86]$. The relationship between single-molecule and structural data provide opportunities to answer intriguing questions. The ribosome field continues to leverage the relationship to elucidate the structure and flexibility of this macromolecular machine.

This dissertation focuses on expanding in the area in-between single-molecule experiments of the ribosome in solution and global structural data of the frozen ribosome. Cryo-EM uses temperature to freeze the ribosome and x-ray crystallography uses steric hindrance to cement the ribosome. Concern arises when dynamics and flexibility of the ribosome are charted to structural conformations obtained by these techniques. Some functions that are known to be manipulated by small forces are unable to make the leap from the single-molecule flexibility and dynamics observed to the structures found of the frozen ribosome [33, 79]. The work in this dissertation develops and applies techniques to monitor the flexible ribosome in solution as it undertakes complex roles in producing the proteome with a focus on gaining global structural information of the ribosome in solution. Expanded roles of the ribosome that are dictated by small forces, specifically frameshifting and co-translational translocation, are investigated. The work nestles and relates the structure of the ribosome in solution to both frozen structures and dynamic measurements, effectively bridging the two data types.

\section{References}

[1] D E Agafonov, V A Kolb, and A S Spirin. "Ribosome-associated protein that inhibits translation at the aminoacyl-tRNA binding stage." eng. In: $E M B O$ 
reports 2.5 (May 2001), pp. 399-402. ISSN: 1469-221X (Print). DOI: 10.1093/ embo-reports/kve091.

[2] John F Atkins et al. "Ribosomal frameshifting and transcriptional slippage: From genetic steganography and cryptography to adventitious use." eng. In: Nucleic acids research 44.15 (Sept. 2016), pp. 7007-7078. ISSN: 1362-4962 (Electronic). DOI: 10.1093/nar/gkw530.

[3] Bertrand Beckert et al. "Structure of a hibernating 100S ribosome reveals an inactive conformation of the ribosomal protein S1". In: Nature Microbiology 3.10 (2018), pp. 1115-1121. ISSN: 2058-5276. DOI: 10.1038/s41564-018-02370. URL: https://doi.org/10.1038/s41564-018-0237-0.

[4] Jose Borreguero et al. "idpflex: Analysis of Intrinsically Disordered Proteins by Comparing Simulations to Small Angle Scattering Experiments". In: Journal of Open Source Software 3.32 (Dec. 11, 2018), p. 1007. ISSN: 2475-9066. DOI: 10.21105/joss.01007, URL: http://dx.doi.org/10.21105/joss.01007.

[5] Zimei Bu, Ligong Wang, and Debra A Kendall. "Nucleotide Binding Induces Changes in the Oligomeric State and Conformation of Sec A in a Lipid Environment: A Small-angle Neutron-scattering Study". In: Journal of Molecular Biology 332.1 (2003), pp. 23-30. ISSN: 0022-2836. DOI: https://doi .org/10. 1016/S0022-2836(03) 00840-4. URL: http: / / www . sciencedirect . com / science/article/pii/S0022283603008404.

[6] Jillian Chase et al. "Mechanisms of opening and closing of the bacterial replicative helicase". In: eLife 7 (2018), pp. 1-42. ISSN: 2050084X. DOI: 10 . 7554 / eLife.41140, 
[7] Jin Chen et al. "Dynamic pathways of -1 translational frameshifting". In: Nature 512.7514 (Aug. 2014), pp. 328-332. ISSN: 0028-0836. DOI: $10.1038 /$ nature13428, URL: http://europepmc.org/articles/PMC4472451.

[8] Jin Chen et al. "Dynamic pathways of -1 translational frameshifting". In: Nature 512.7514 (Aug. 2014), pp. 328-332. ISSN: 0028-0836. DOI: 10.1038/ nature13428, URL: http://europepmc.org/articles/PMC4472451.

[9] Peter V Cornish et al. "Following movement of the L1 stalk between three functional states in single ribosomes". In: Proceedings of the National Academy of Sciences 106.8 (2009), pp. 2571-2576. ISSN: 0027-8424. DOI: 10.1073/pnas. 0813180106. URL: https://www.pnas .org/content/106/8/2571

[10] Peter V. Cornish et al. "Spontaneous Intersubunit Rotation in Single Ribosomes". In: Molecular Cell (2008). ISSN: 10972765. DOI: 10 .1016/ j . molcel. 2008.05.004, arXiv: NIHMS150003,

[11] Joseph E Curtis et al. "SASSIE: A program to study intrinsically disordered biological molecules and macromolecular ensembles using experimental scattering restraints". In: Computer Physics Communications 183.2 (2012), pp. 382389. ISSN: 0010-4655. DOI: https : // doi . org / 10 . 1016/ j . cpc . 2011 . 09.010. URL: http://wWw . sciencedirect .com/science/article/pii/ S0010465511003286.

[12] Ali Dashti et al. "Trajectories of the ribosome as a Brownian nanomachine". In: Proceedings of the National Academy of Sciences 111.49 (2014), pp. 1749217497. ISSN: 0027-8424. DOI:10.1073/pnas .1419276111. URL: https ://www . pnas.org/content/111/49/17492. 
[13] Gabriel Demo et al. "Mechanism of ribosome rescue by ArfA and RF2". In: eLife 6 (2017). Ed. by Rachel Green, e23687. ISSN: 2050-084X. DOI: 10 .7554/ eLife.23687. URL: https://doi.org/10.7554/eLife.23687

[14] Amédée Des Georges et al. "Structure of the mammalian ribosomal pretermination complex associated with eRF1 $\bullet$ RF3 $\bullet$ GDPNP". In: Nucleic Acids Research 42.5 (2014), pp. 3409-3418. ISSN: 13624962. DOI: 10 . 1093/nar / gkt1279,

[15] Jack A Dunkle et al. "Structures of the bacterial ribosome in classical and hybrid states of tRNA binding." eng. In: Science (New York, N.Y.) 332.6032 (May 2011), pp. 981-984. ISSN: 1095-9203 (Electronic). DOI: 10 . 1126 / science . 1202692 .

[16] Shu Feng, Yun Chen, and Yong Gui Gao. "Crystal Structure of 70S Ribosome with Both Cognate tRNAs in the E and P Sites Representing an Authentic Elongation Complex". In: PLoS ONE 8.3 (2013), pp. 1-11. ISSN: 19326203. DOI: 10.1371/journal.pone.0058829

[17] Joachim Frank. "New Opportunities Created by Single-Particle Cryo-EM: The Mapping of Conformational Space". In: Biochemistry 57.6 (Feb. 2018), p. 888. ISSN: 0006-2960. DOI: 10 . 1021/acs . biochem . 8b00064. URL: https : //doi.org/10.1021/acs.biochem.8b00064.

[18] Daniel Franke and Dmitri I Svergun. "DAMMIF, a program for rapid abinitio shape determination in small-angle scattering." eng. In: Journal of applied crystallography 42.Pt 2 (Apr. 2009), pp. 342-346. ISSN: 0021-8898 (Print). DOI: $10.1107 / \mathrm{S} 0021889809000338$. 
[19] Frank Gabel. "Small-Angle Neutron Scattering for Structural Biology of ProteinRNA Complexes." eng. In: Methods in enzymology 558 (2015), pp. 391-415. ISSN: 1557-7988 (Electronic). DOI: 10.1016/bs .mie.2015.02.003.

[20] Haixiao Gao et al. "Study of the Structural Dynamics of the E. coli $70 S$ Ribosome Using Real-Space Refinement Georgia Institute of Technology". In: Cell 113 (2003), pp. 789-801.

[21] Amédée des Georges et al. "Structural Basis for Gating and Activation of RyR1". eng. In: Cell 167.1 (Sept. 2016), 145-157.e17. ISSN: 1097-4172. DOI: 10. 1016/j.cell.2016.08.075. URL: https://www.ncbi.nlm.nih.gov/pubmed/ 27662087\%20https://www.ncbi.nlm.nih.gov/pmc/articles/PMC5142848/.

[22] David P Giedroc and Peter V Cornish. "Frameshifting RNA pseudoknots: Structure and mechanism". In: Virus Research 139.2 (2009), pp. 193-208. ISSN: 0168-1702. DOI: https://doi .org/10.1016/j .virusres .2008.06.008, URL: http://www.sciencedirect.com/science/article/pii/S0168170208002323

[23] Veit Goder and Martin Spiess. “Molecular mechanism of signal sequence orientation in the endoplasmic reticulum". In: The EMBO Journal 22.14 (July 2003), pp. 3645-3653. ISSN: 0261-4189. DOI: 10 . 1093/ emboj / cdg361. URL: https://doi.org/10.1093/emboj/cdg361.

[24] Thomas D Grant. "Ab initio electron density determination directly from solution scattering data". In: Nature Methods 15 (Jan. 2018), p. 191. URL: https: //doi.org/10.1038/nmeth.4581\%20http://10.0.4.14/nmeth. 4581\% 20https://www .nature.com/articles/nmeth.4581\%7B\%5C\#\%7Dsupplementaryinformation,

[25] Claudio O. Gualerzi and Cynthia L. Pon. "Initiation of mRNA translation in bacteria: structural and dynamic aspects". In: Cellular and Molecular Life 
Sciences 72.22 (Nov. 2015), pp. 4341-4367. ISSN: 1420-9071. DOI: $10.1007 /$ s00018-015-2010-3. URL: https://doi.org/10.1007/s00018-015-2010-3.

[26] Guinier, André. "La diffraction des rayons $X$ aux très petits angles : application à l'étude de phénomènes ultramicroscopiques". In: Ann. Phys. 11.12 (1939), pp. 161-237. DOI: 10 . 1051/anphys /193911120161. URL: https : // doi.org/10.1051/anphys/193911120161

[27] Boualem Hammouda. "Probing Nanoscale Structures - the SANS Toolbox". In: (2008).

[28] Jeffrey L Hansen et al. "Structural insights into peptide bond formation." eng. In: Proceedings of the National Academy of Sciences of the United States of America 99.18 (Sept. 2002), pp. 11670-11675. ISSN: 0027-8424 (Print). DOI: 10. 1073/pnas.172404099.

[29] S Hansen. "Calculation of small-angle scattering profiles using Monte Carlo simulation". In: Journal of Applied Crystallography 23.4 (Aug. 1990), pp. 344346. ISSN: 1600-5767. DOI: 10.1107/S0021889890002801. URL: https://doi. org/10.1107/S0021889890002801.

[30] Yaser Hashem et al. "Structure of the mammalian ribosomal 435 preinitiation complex bound to the scanning factor DHX29". In: Cell 153.5 (May 2013), pp. 1108-1119. ISSN: 1097-4172. DOI: 10 . 1016/j . cell . 2013 . 04 . 036. URL: https://www.ncbi.nlm.nih.gov/pubmed/23706745\%20https://www.ncbi. nlm.nih.gov/pmc/PMC3730827/

[31] Ruth E Hoffmeyer, Peter Bündgen, and Ajit J Thakkar. "Cross sections for x-ray and high-energy electron scattering by small molecules". In: Journal of Physics B: Atomic, Molecular and Optical Physics 31.16 (Aug. 1998), pp. 36753692. DOI: 10.1088/0953-4075/31/16/019. URL: https://doi .org/10. 
$1088 \% 7 \mathrm{~B} \% 5 \mathrm{C} \% \%$ D2F0953-4075\%7B $\% 5 \mathrm{C} \% \%$ D2F31\%7B $\% 5 \mathrm{C} \% \% 7 \mathrm{D} 2 \mathrm{~F} 16 \% 7 \mathrm{~B} \% 5 \mathrm{C} \%$ $\% 7 \mathrm{D} 2 \mathrm{~F} 019$.

[32] Samuel Hong et al. "Mechanism of tRNA-mediated +1 ribosomal frameshifting". In: Proceedings of the National Academy of Sciences 115.44 (2018), pp. 1122611231. ISSN: 0027-8424. DOI: $10.1073 /$ pnas . 1809319115

[33] Nurzian Ismail et al. "Charge-driven dynamics of nascent-chain movement through the SecYEG translocon". In: Nature Structural EAmp; Molecular Biology 22 (Jan. 2015), p. 145. URL: https : // doi . org/10 . 1038/nsmb . 2940\% 20http://10.0.4.14/nsmb. $2940 \% 20 \mathrm{https://www.nature.com/articles/}$ nsmb. $2940 \% 7 \mathrm{~B} \% 5 \mathrm{C} \# \%$ 7Dsupplementary-information

[34] Koreaki Ito et al. "Nascentome analysis uncovers futile protein synthesis in Escherichia coli." eng. In: PloS one 6.12 (2011), e28413. ISSN: 1932-6203 (Electronic). DOI: $10.1371 /$ journal.pone.0028413.

[35] Tyler Jacks et al. "Signals for ribosomal frameshifting in the rous sarcoma virus gag-pol region". In: Cell 55.3 (1988), pp. 447-458. ISSN: 0092-8674. DOI: http://dx.doi.org/10.1016/0092-8674(88)90031-1. URL: http://www . sciencedirect.com/science/article/pii/0092867488900311.

[36] David A Jacques and Jill Trewhella. "Small-angle scattering for structural biology-expanding the frontier while avoiding the pitfalls." eng. In: Protein science : a publication of the Protein Society 19.4 (Apr. 2010), pp. 642-657. ISSN: 1469-896X (Electronic). DOI: 10.1002/pro.351.

[37] B Jacrot. "The study of biological structures by neutron scattering from solution". In: Reports on Progress in Physics 39.10 (1976), pp. 911-953. ISSN: 00344885. DOI: 10 .1088/0034-4885/39/10/001. URL: http://dx . doi .org/10. 1088/0034-4885/39/10/001. 
[38] Hong Jin, Ann C Kelley, and V Ramakrishnan. "Crystal structure of the hybrid state of ribosome in complex with the guanosine triphosphatase release factor 3." eng. In: Proceedings of the National Academy of Sciences of the United States of America 108.38 (Sept. 2011), pp. 15798-15803. ISSN: 1091-6490 (Electronic). DOI: 10.1073/pnas.1112185108.

[39] Patricia Julián et al. "Structure of ratcheted ribosomes with tRNAs in hybrid states". In: Proceedings of the National Academy of Sciences of the United States of America 105.44 (2008), pp. 16924-16927. ISSN: 00278424. DOI: 10.1073/pnas. 0809587105 .

[40] Kenneth C Keiler. "Mechanisms of ribosome rescue in bacteria". In: Nature Reviews Microbiology 13 (Apr. 2015), p. 285. URL: https://doi.org/10.1038/ nrmicro3438\%20http://10.0.4.14/nrmicro3438.

[41] Serdal Kirmizialtin et al. Using molecular simulation to model high-resolution cryo-EM reconstructions. 1st ed. Vol. 558. 1. Elsevier Inc., 2015, pp. 497-514. DOI: 10.1016/bs.mie.2015.02.011. URL: http://dx.doi.org/10.1016/bs. mie.2015.02.011.

[42] Hans-Georg Koch et al. "In Vitro Studies with Purified Components Reveal Signal Recognition Particle (SRP) and SecA/SecB as Constituents of Two Independent Protein-targeting Pathways of Escherichia coli". In: Molecular Biology of the Cell 10.7 (1999), pp. 2163-2173. ISSN: 1059-1524. URL: http://www. ncbi.nlm.nih.gov/pmc/articles/PMC25430/.

[43] Patrick Kuhn et al. "Ribosome binding induces repositioning of the signal recognition particle receptor on the translocon." eng. In: The Journal of cell biology 211.1 (Oct. 2015), pp. 91-104. ISSN: 1540-8140 (Electronic). DOI: 10 . $1083 / j \mathrm{jcb} .201502103$. 
[44] Kathrin Lang et al. "The role of 235 ribosomal RNA residue A2451 in peptide bond synthesis revealed by atomic mutagenesis." eng. In: Chemistry $\mathcal{E}$ biology 15.5 (May 2008), pp. 485-492. ISSN: 1074-5521 (Print). DOI: 10.1016/ j.chembiol.2008.03.014.

[45] Martin Laurberg et al. "Structural basis for translation termination on the 705 ribosome." eng. In: Nature 454.7206 (Aug. 2008), pp. 852-857. ISSN: 1476-4687 (Electronic). DOI: 10.1038/nature07115.

[46] J S Lodmell and A E Dahlberg. "A conformational switch in Escherichia coli $16 \mathrm{~S}$ ribosomal RNA during decoding of messenger RNA." eng. In: Science (New York, N.Y.) 277.5330 (Aug. 1997), pp. 1262-1267. ISSN: 0036-8075 (Print). DOI: $10.1126 /$ science. 277.5330 .1262 .

[47] Ottilie von Loeffelholz et al. "Ribosome-SRP-FtsY cotranslational targeting complex in the closed state." eng. In: Proceedings of the National Academy of Sciences of the United States of America 112.13 (Mar. 2015), pp. 3943-3948. ISSN: 1091-6490 (Electronic). DOI: 10.1073/pnas. 1424453112.

[48] Anna B Loveland and Andrei A Korostelev. "Structural dynamics of protein S1 on the 70S ribosome visualized by ensemble cryo-EM". In: Methods (San Diego, Calif.) 137 (Mar. 2018), pp. 55-66. ISSN: 1095-9130. DOI: $10.1016 / \mathrm{j}$. ymeth . 2017 .12 .004, URL: https : // www . ncbi .nlm . nih . gov/pubmed/ 29247757\%20https://www.ncbi.nlm.nih.gov/pmc/PMC5866760/.

[49] Justine Mailliot and Franck Martin. "Viral internal ribosomal entry sites: four classes for one goal." eng. In: Wiley interdisciplinary reviews. RNA 9.2 (Mar. 2018). ISSN: 1757-7012 (Electronic). DOI: 10.1002/wrna.1458.

[50] Soneya Majumdar et al. "Disrupting domain-domain interactions is indispensable for EngA-ribosome interactions". In: Biochimica et Biophysica Acta - 
Proteins and Proteomics 1865.3 (2017), pp. 289-303. ISSN: 18781454. DOI: 10. 1016/j . bbapap . 2016 .12 .005. URL: http : / / dx . doi .org/10 .1016/j . bbapap.2016.12.005.

[51] R P May, K Ibel, and J Haas. "The forward scattering of cold neutrons by mixtures of light and heavy water". In: Journal of Applied Crystallography 15.1 (Feb. 1982), pp. 15-19. DOI: 10.1107/S0021889882011285. URL: https : // doi.org/10.1107/S0021889882011285

[52] Mickey R Miller et al. "The role of SmpB and the ribosomal decoding center in licensing tmRNA entry into stalled ribosomes". eng. In: RNA (New York, N.Y.) 17.9 (Sept. 2011), pp. 1727-1736. ISSN: 1469-9001. DOI: 10.1261/ rna. 2821711. URL: https : //wWw . ncbi.nlm . nih.gov/pubmed/21795410\% 20https://www.ncbi.nlm.nih.gov/pmc/articles/PMC3162337/

[53] Pohl Milón et al. "Real-time assembly landscape of bacterial $30 \mathrm{~S}$ translation initiation complex". In: Nature Structural EAmp; Molecular Biology 19 (May 2012), p. 609. URL: https://doi.org/10.1038/nsmb.2285\%20http://10.0. 4.14/nsmb. $2285 \% 20$ https://www .nature.com/articles/nsmb. $2285 \% 7 \mathrm{~B} \% 5 \mathrm{C \#}$ \%7Dsupplementary-information.

[54] Pete Moore. "The GTPase switch in ribosomal translocation". eng. In: Journal of biology 4.2 (2005), p. 7. ISSN: 1475-4924. DOI: 10.1186/jbiol28. URL: https : //www.ncbi.nlm.nih.gov/pubmed/15985152\%20https://www.ncbi.nlm. nih.gov/pmc/articles/PMC1176000/

[55] M Müller and G Blobel. "In vitro translocation of bacterial proteins across the plasma membrane of Escherichia coli". In: Proceedings of the National Academy of Sciences of the United States of America 81.23 (1984), pp. 7421-7425. ISSN: 
0027-8424 1091-6490. URL: http://www.ncbi.nlm.nih.gov/pmc/articles/ PMC392158/,

[56] Knud H Nierhaus and Denis L Lafontaine. Ribosome Assembly. Aug. 2004. DOI: doi : 10 . 1002/3527603433 .ch3, URL: https : // doi . org/10 . 1002/ $3527603433 . \mathrm{ch} 3$,

[57] Poul Nissen et al. "The Structural Basis of Ribosome Activity in Peptide Bond Synthesis". In: Science 289.5481 (Aug. 2000), 920 LP -930. DOI: 10.1126/ science.289.5481.920. URL: http://science.sciencemag.org/content/ 289/5481/920. abstract.

[58] Thomas R Noriega et al. "Signal Recognition Particle-ribosome Binding Is Sensitive to Nascent Chain Length". In: Journal of Biological Chemistry 289.28 (July 2014), pp. 19294-19305. URL: http://www . jbc.org/content/289/28/ 19294.abstract.

[59] Trushar R Patel et al. "Structural studies of RNA-protein complexes: A hybrid approach involving hydrodynamics, scattering, and computational methods". In: Methods 118-119 (2017), pp. 146-162. ISSN: 1046-2023. DOI: https:// doi.org/10.1016/j.ymeth.2016.12.002, URL:http://www.sciencedirect. com/science/article/pii/S1046202316304753

[60] Xiaohui Qu et al. "The ribosome uses two active mechanisms to unwind messenger RNA during translation". In: Nature 475 (July 2011), p. 118. URL: https://doi .org/10.1038/nature10126\%20http://10.0.4.14/ nature $10126 \% 20 \mathrm{https}: / /$ ww . nature . com/articles / nature $10126 \% 7 \mathrm{~B} \%$ 5C\#\%7Dsupplementary-information

[61] Anand Ramanathan, G. Brett Robb, and Siu-Hong Chan. "mRNA capping: biological functions and applications". In: Nucleic Acids Research 44.16 (June 
2016), pp. 7511-7526. ISSN: 0305-1048. DOI: 10.1093/nar/gkw551. eprint: http : / / oup .prod.sis . lan/nar/article-pdf /44/16/7511/7431314/ gkw551.pdf. URL: https://dx.doi.org/10.1093/nar/gkw551

[62] Robert P Rambo and John A Tainer. "Accurate assessment of mass, models and resolution by small-angle scattering". In: Nature 496 (Apr. 2013), p. 477. URL: https://doi .org/10.1038/nature12070\%20http://10.0.4.14/ nature $12070 \% 20 \mathrm{https}$ ://www . nature. com/articles/nature $12070 \% 7 \mathrm{~B} \% 5 \mathrm{C \#}$ \%7Dsupplementary-information.

[63] Robert P Rambo and John A Tainer. "Bridging the solution divide: comprehensive structural analyses of dynamic RNA, DNA, and protein assemblies by small-angle X-ray scattering". In: Current Opinion in Structural Biology 20.1 (2010), pp. 128-137. ISSN: 0959-440X. DOI: https : // doi . org/10 . 1016/ j.sbi .2009.12.015. URL: http://www . sciencedirect .com/science/ article/pii/S0959440X09002036.

[64] Rahul Roy, Sungchul Hohng, and Taekjip Ha. "A practical guide to singlemolecule FRET". eng. In: Nature methods 5.6 (June 2008), pp. 507-516. ISSN: 1548-7105. DOI: 10.1038/nmeth.1208, URL: https ://www . ncbi .nlm .nih . gov/pubmed/18511918\%20https://www.ncbi.nlm.nih.gov/pmc/articles/ PMC3769523/.

[65] Ekaterina Samatova et al. "High-efficiency translational bypassing of noncoding nucleotides specified by mRNA structure and nascent peptide". In: Nature Communications 5 (2014), pp. 1-10. ISSN: 20411723. DOI: 10 . $1038 /$ ncomms5459. URL: http://dx.doi.org/10.1038/ncomms5459.

[66] Martina Sauert, Hannes Temmel, and Isabella Moll. "Heterogeneity of the translational machinery: Variations on a common theme." eng. In: Biochimie 
114 (July 2015), pp. 39-47. ISSN: 1638-6183 (Electronic). DOI: $10.1016 / \mathrm{j}$. biochi.2014.12.011.

[67] T Martin Schmeing and V Ramakrishnan. "What recent ribosome structures have revealed about the mechanism of translation". In: Nature 461 (Oct. 2009), p. 1234. URL: https://doi .org/10.1038/nature08403\%20http://10.0.4. $14 /$ nature08403.

[68] Varley F Sears. "Neutron scattering lengths and cross sections". In: Neutron News 3.3 (Jan. 1992), pp. 26-37. ISSN: 1044-8632. DOI: 10.1080/10448639208218770. URL: https://doi.org/10.1080/10448639208218770.

[69] Sunil Shetty et al. "Two highly conserved features of bacterial initiator tRNAs license them to pass through distinct checkpoints in translation initiation". In: Nucleic acids research 45.4 (Feb. 2017), pp. 2040-2050. ISSN: 13624962. DOI: 10 . 1093/nar/gkw854. URL: https ://www .ncbi.nlm .nih.gov/ pubmed/28204695\%20https://www.ncbi.nlm.nih.gov/pmc/PMC5389676/.

[70] Yoshihiro Shimizu. "ArfA Recruits RF2 into Stalled Ribosomes". In: Journal of Molecular Biology 423.4 (2012), pp. 624-631. ISSN: 0022-2836. DOI: https:// doi.org/10.1016/j.jmb.2012.08.007, URL: http://www.sciencedirect. com/science/article/pii/S0022283612006699.

[71] John Shine and Lynn Dalgarno. “Terminal-Sequence Analysis of Bacterial Ribosomal RNA". In: European Journal of Biochemistry 57.1 (1975), pp. 221230. DOI: $10.1111 / \mathrm{j} .1432-1033$.1975 . tb02294 . x. URL: https : //febs . onlinelibrary.wiley.com/doi/abs/10.1111/j.1432-1033.1975.tb02294. X. 
[72] D I Svergun. "Mathematical methods in small-angle scattering data analysis". In: Journal of Applied Crystallography 24.5 (Oct. 1991), pp. 485-492. DOI: 10.1107/S0021889891001280. URL:https://doi .org/10.1107/S0021889891001280.

[73] D I Svergun. "Restoring low resolution structure of biological macromolecules from solution scattering using simulated annealing". eng. In: Biophysical journal 76.6 (June 1999), pp. 2879-2886. ISSN: 0006-3495. DOI: 10.1016/S00063495(99) 77443-6. URL:https://www.ncbi.nlm.nih.gov/pubmed/10354416\% 20https://www.ncbi.nlm.nih.gov/pmc/articles/PMC1300260/.

[74] D I Svergun et al. "Protein hydration in solution: Experimental observation by x-ray and neutron scattering". In: Proceedings of the National Academy of Sciences 95.5 (1998), pp. 2267-2272. ISSN: 0027-8424. DOI: 10.1073/pnas . 95. 5.2267. URL: https://www.pnas.org/content/95/5/2267.

[75] D Svergun, C Barberato, and M H J Koch. "CRYSOL a Program to Evaluate X-ray Solution Scattering of Biological Macromolecules from Atomic Coordinates". In: Journal of Applied Crystallography 28.6 (Dec. 1995), pp. $768-$ 773. DOI: 10.1107/S0021889895007047. URL: https ://doi .org/10 .1107/ S0021889895007047.

[76] Dmitri I Svergun et al. Small Angle X-Ray and Neutron Scattering from Solutions of Biological Macromolecules. eng. Oxford: Oxford University Press, 2013, p. 368. ISBN: 9780199639533 . DOI: 10 . 1093/acprof : oso/9780199639533. 001.0001 . URL: https : //www . oxfordscholarship . com/10 . 1093/acprof : oso/9780199639533.001.0001/acprof-9780199639533.

[77] J R Thom and L L Randall. "Role of the leader peptide of maltose-binding protein in two steps of the export process". In: Journal of Bacteriology 170.12 
(1988), pp. 5654-5661. ISSN: 0021-9193 1098-5530. URL: http://www . ncbi . nlm.nih.gov/pmc/articles/PMC211665/

[78] Jill Trewhella et al. "2017 publication guidelines for structural modelling of small-angle scattering data from biomolecules in solution: an update." eng. In: Acta crystallographica. Section D, Structural biology 73.Pt 9 (Sept. 2017), pp. 710-728. ISSN: 2059-7983 (Electronic). DOI: 10.1107/S2059798317011597

[79] Z Tsuchihashi and P O Brown. "Sequence requirements for efficient translational frameshifting in the Escherichia coli dnaX gene and the role of an unstable interaction between tRNA(Lys) and an AAG lysine codon." eng. In: Genes $\mathcal{E}$ development 6.3 (Mar. 1992), pp. 511-519. ISSN: 0890-9369 (Print). DOI: 10.1101/gad.6.3.511.

[80] Masami Ueta et al. "Ribosome binding proteins YhbH and YfiA have opposite functions during 1005 formation in the stationary phase of Escherichia coli." eng. In: Genes to cells : devoted to molecular \& cellular mechanisms 10.12 (Dec. 2005), pp. 1103-1112. ISSN: 1356-9597 (Print). DOI: $10.1111 /$ j . 1365$2443.2005 .00903 \cdot x$.

[81] Masami Ueta et al. "Role of HPF (hibernation promoting factor) in translational activity in Escherichia coli." eng. In: Journal of biochemistry 143.3 (Mar. 2008), pp. 425-433. ISSN: 0021-924X (Print). DOI: 10.1093/jb/mvm243.

[82] A Wada et al. "Structure and probable genetic location of a "ribosome modulation factor" associated with 100S ribosomes in stationary-phase Escherichia coli cells." eng. In: Proceedings of the National Academy of Sciences of the United States of America 87.7 (Apr. 1990), pp. 2657-2661. ISSN: 0027-8424 (Print). DOI: $10.1073 /$ pnas .87.7.2657. 
[83] Joseph M Watts et al. "Architecture and secondary structure of an entire HIV1 RNA genome". In: Nature 460.7256 (Aug. 2009), pp. 711-716. ISSN: 00280836. URL: http://dx . doi .org/10 .1038/nature08237\%20http://www . nature.com/nature/journal/v460/n7256/suppinfo/nature08237\%7B\%5C_ $\%$ DDS1.html.

[84] Joshua S Weinger et al. "Substrate-assisted catalysis of peptide bond formation by the ribosome." eng. In: Nature structural \& molecular biology 11.11 (Nov. 2004), pp. 1101-1106. ISSN: 1545-9993 (Print). DOI: 10.1038/nsmb841.

[85] Thomas Welte et al. "Promiscuous targeting of polytopic membrane proteins to SecYEG or YidC by the Escherichia coli signal recognition particle". eng. In: Molecular biology of the cell 23.3 (Feb. 2012), pp. 464-479. ISSN: 1939-4586. DOI: 10.1091/mbc.E11-07-0590. URL: https ://www .ncbi .nlm .nih .gov/ pubmed / 22160593\%20https : //www .ncbi.nlm.nih . gov/pmc/articles / PMC3268725/.

[86] Jin-Der Wen et al. "Following translation by single ribosomes one codon at a time". In: Nature 452.7187 (Apr. 2008), pp. 598-603. ISSN: 0028-0836. DOI: 10.1038/nature06716. URL: http://europepmc.org/articles/PMC2556548.

[87] Paul C. Whitford and Karissa Y. Sanbonmatsu. "Simulating movement of tRNA through the ribosome during hybrid-state formation". In: Journal of Chemical Physics 139.12 (2013), pp. 1-9. ISSN: 00219606. DOI: 10 . 1063/1. 4817212 ,

[88] Zht Cheng Wu et al. "Competitive Binding of the SecA ATPase and Ribosomes to the SecYEG Translocon". In: Journal of Biological Chemistry 287.11 (Mar. 2012), pp. 7885-7895. URL: http: //www · jbc . org/content/287/11/ 7885.abstract. 
[89] Shannon Yan et al. "Ribosome Excursions during mRNA Translocation Mediate Broad Branching of Frameshift Pathways". In: Cell 160.5 (2015), pp. 870881. ISSN: 10974172. DOI: $10.1016 /$ j.cell.2015.02.003.

[90] Bin Zhang and Thomas F 3rd Miller. "Long-timescale dynamics and regulation of Sec-facilitated protein translocation." eng. In: Cell reports 2.4 (Oct. 2012), pp. 927-937. ISSN: 2211-1247 (Electronic). DOI: 10 . 1016/ j . celrep. 2012.08 .039

[91] Jun Zhang et al. "Mechanisms of ribosome stalling by SecM at multiple elongation steps". In: eLife 4 (2015). Ed. by Sjors H W Scheres, e09684. ISSN: 2050084X. DOI: 10.7554/eLife.09684. URL: https://doi.org/10.7554/eLife. 09684 .

[92] Yan Zhang et al. "Alternative Mode of E-Site tRNA Binding in the Presence of a Downstream mRNA Stem Loop at the Entrance Channel". In: Structure 26.3 (2018), 437-445.e3. ISSN: 18784186. DOI: 10.1016/j . str.2018.01.013. URL: https://doi.org/10.1016/j.str.2018.01.013. 


\section{Ribosome Purification and Characterization}

The ribosomes resulting from novel purifications are characterized in this chapter and utilized in Chapters 4 and 5. Section 2.1 introduces methods of ribosome purification and details a novel purification method that was developed. Section 2.2 introduces three characterization techniques and describes their application to ribosomes made by the novel purification technique described in Section 2.1.3.

\subsection{Ribosome Purification Methods}

Structure, dynamics, and in vitro synthesis work all require active ribosomes and the purification method can depend on the use of the ribosome. Due to their complexity, the purification of ribosomes differs from procedures commonly used for protein purification and RNA purification procedures. Studies that utilize or observe active translation-ready ribosomes will likely use cells that have been harvested during log-phase of growth and slowly cooled. Log-phase growth happens in high nutrient situations when cells are growing and dividing rapidly. Often ribosomes are cooled slowly to $4^{\circ} \mathrm{C}$ to allow polysomes to shed mRNA, this creates a large amount of active and free $70 S$ that can be purified [2]. After cells are harvested, cooled, and pelleted, they are broken open to access the ribosomes in the cytoplasm.

\subsubsection{Classical Purification}

By the early 1970s, about 15 years after Palade first discovered the ribosome, a standard method of ribosome purification was established [24]. Throughout the protocol the buffer is at $\mathrm{pH} 7.5$, usually using 10 to $20 \mathrm{mM}$ Tris with each step performed at $4 \pm 4^{\circ} \mathrm{C}$. After cell lysis, centrifugation with a fixed-angle rotor for

10 to 15 minutes at around 5,000 g will pellet the cell membrane and heavy ag- 
gregations at the bottom of the tube. A second spin of the supernatant at 30,000 $\mathrm{g}$ for 30 minutes will produce a cell extract known as S30 [6], which is often used for in vitro translation reactions. When pure 70S, or $50 S$ and $30 S$ subunits are the objective, S30 is layered on top of a $1.1 \mathrm{M}$ sucrose and the salt concentration is increased to $500 \mathrm{mM}$. Any protein that sticks to the ribosome above $500 \mathrm{mM}$ is defined as a ribosomal protein [1]. This is spun down for about 16-18 hours at 120,00 g. The pellet can be dialyzed and can be used for in vitro synthesis; the extract is named S100. If pure 70S ribosomes are wanted, the S100 is concentrated and layered on top of a sucrose gradient. A sucrose gradient is a linear gradient of sucrose density in solution, and physical disruption of the gradient will ruin future steps and ribosome purity. This point is a branch point in the protocol with the path forward depending on whether the desired yield is 70S ribosomes or 30S and $50 \mathrm{~S}$ ribosomal subunits. The former is called tight couple purification because the subunits are tightly bound to each other and thus the 705 will be purified, while the latter is referred to as subunit preparation. The main difference is that the sucrose gradient is from $10-30 \%$ and the magnesium levels are dropped to around $1 \mathrm{mM}$ in subunit prep [11]. Tight couple ribosomes will be layered on a 10-35\% sucrose cushion with a Magnesium concentration equal to or greater than $10 \mathrm{mM}$ [24, 11, 2]. These sucrose gradients are spun at 53,000 $\mathrm{g}$ for 13 hours or $83,000 \mathrm{~g}$ for 14 hours for tight-coupled 70S and subunit preparation, respectively. With minimal disturbance to the gradient after centrifugation, the layers of the gradients are fractionated. The 30S, 50S and 70S will appear in the fractions where the sucrose density equal to particle density because, during centrifugation, the $g$ forces applied to a particle are negated by buoyant forces of a sucrose layer that is more dense. In as much as the classic method employs centrifugation as the main technique in purification, the stokes coefficients are used for identification purposes of 
the complex and components. With these purified complexes, it has been shown that the ribosomal proteins and the rRNA components can be separated from each other and then reconstituted/assembled in vitro [18]. This feature has been used for specific deuteration labeling of rRNA and ribosomal proteins in Small Angle Neutron Scattering experiments [26, 27], but it has been shown to decrease activity by up to $50 \%$ [18].

\subsubsection{Novel Purification Techniques}

The classical purification protocol takes a tour de force to perform, so novel methods have been developed that decrease labor, time, and expenses as well as decrease the $\mathrm{g}$ forces the ribosomes experience during the process. Size exclusion, also called gel filtration, chromatography has been applied to purify the ribosome [13], although it has not been widely utilized due to a decrease seen in ribosome activity after purification by this method. In contrast to size exclusion chromatography, affinity chromatography purification methods have shown promise in producing highly active ribosomes [22, 30]. Affinity chromatography has been used for the specific selection of mutated ribosomes. For example, ribosomes with mutated rRNA can be transcribed with an MS2 tag that allows for isolation of the mutated ribosomes over the wild-type [30]. An affinity method that has gained wide use is his-tagging of the bL7/bL12 protein and purifying on a nickel column [9. 22]. The latter is applied in the novel purification detailed below.

\subsubsection{Application of a Novel Purification Method}

The work done in Chapters 3 and 5 use ribosomes that are purified using the novel technique described here and the resulting ribosomes are characterized in Section 2.2. BW 2113 E. coli cells that have been modified to have a his-tag on the bL12/bL7 
[9] were grown in a Bio-Rad fermentor in minimal media [28] and harvested once the cells reached an optical density of 11 AD600 by chilling and shaking the culture on ice. Cells were pelleted at $4^{\circ} \mathrm{C}$. Ribosomes were purified using nickel chromatography [9], fractions from the A260 peak were selected, pooled, and concentrated via spinning in a Millipore $100 \mathrm{MWCO}$ centricon tube at $3000 \mathrm{rgf}$. $250 \mathrm{ul}$ of sample was layered onto a $30 \mathrm{ml} 10-35 \%$ sucrose gradient of 100/10 buffer (20 mM Tris $\mathrm{pH}$ 7.6, $10 \mathrm{mM} \mathrm{MgCl}_{2}, 100 \mathrm{mM} \mathrm{NH}_{4} \mathrm{Cl}$ ). The gradient was spun in a Thermo Scientific AH-629 swinging bucket rotor for 17 hours at 19,000 rpm and was fractionated into $1 \mathrm{ml}$ fractions kept on ice. Fractions were assessed using dynamic light scattering (DLS) and SDS-Polyacrylamide Gel Electrophoresis (SDS-PAGE). Those with a radius of hydration of $15 \pm 2 \mathrm{~nm}$, low polydispersity (as shown by DLS measurements), and with no protein contamination from SDS-PAGE were pooled, concentrated. The ribosomes were then exchanged into a buffer solution of $20 \mathrm{mM}$ HEPES pH 7.5, $6 \mathrm{mM} \mathrm{MgCl} 2,150 \mathrm{mM} \mathrm{NH}_{4} \mathrm{Cl}, 6 \mathrm{mM} \beta \mathrm{ME}, 2 \mathrm{mM}$ Spermidine, and $0.1 \mathrm{mM}$ Spermine and the solution was then flash-frozen in liquid nitrogen. These ribosomes were further characterized with SDS-PAGE, dynamic light scattering, and Negative Stain.

\subsection{Ribosome Characterization Methods}

During and after any purification process, characterization is required to determine the contents of the purified material. The component parts of the ribosome, protein and RNA, can easily be investigated separately using polyacrylamide and agarose electrophoresis methods. These methods report on the weight or degradation of the component parts of the samples, but fail to report holistically on the macromolecular complex. The size, shape, and functionality of the intact com-

plex must be established in order to characterize a ribosome sample. For example, 
the ribosomes purified in Section 2.1.3 showed only 79\% activity compared to the activity of classically purified tight-coupled ribosomes, when compared using a PURExpress transcription translation reaction [23]. Therefore, it is obligatory to use corroborative techniques to reach a complete understanding of the molecule of interest. Three methods of characterization are introduced below and applied to the ribosomes made using the protocol in Section 2.1.3.

\subsubsection{SDS Polyacrylamide Gel Electrophoresis}

In the past, 2-dimensional PAGE has been used to identify ribosomal proteins [17. 19]. In our case, the ribosomal proteins are sufficiently well characterized, that 1dimensional electrophoresis can be used to determine the ribosomal protein presence. Sodium dodecyl sulfate Polyacrylamide Gel Electrophoresis (SDS-PAGE) separates proteins by size by first unfolding and coating the molecules with charge using SDS and then mobilizing protein using electric charge applied to a gel and buffer system. This technique is the tool that is most often used to asses protein size and purity and also in the beginning steps of a blotting procedure or mass spectrometry sample preparation [5]. Here we use SDS-PAGE to assess the protein contents of a ribosome sample. There are 57 proteins on a bacterial $70 S$ ribosome. On the large subunit, there are 36, and the small subunit has 21 proteins. Each protein has a certain affinity for the ribosome, and the binding affinity can depend on environmental factors. For example, S1 is stabilized on the ribosome by $\mathrm{S} 2$ and zinc is involved in the binding [7]. Each time a novel purification method is used, where the environments and buffers vary from those in the classical purification, the purity of the sample and the presence of ribosomal protein can be assessed by SDS-PAGE. The largest ribosomal protein is S1, which is nearly 62 kiloDaltons $(\mathrm{kDa})$ and; all the other ribosomal proteins have a weight of less than $30 \mathrm{kDa}$ so 
anything in this gap or above $62 \mathrm{kDa}$ is a clear contaminant. Often an amino acid chain that is still bound to a tRNA will show up in these regions, which means that the purified ribosomes contain tRNA.

Application of SDS-PAGE 1-Dimensional SDS-PAGE was used to assess the ribosomes purified using the novel method described in 2.1.3 (A, Figure 7). Ribosomes were run on a $4-10 \%$ gel along with tight-coupled ribosomes (E. coli ribosomes (B, Figure 7), New England Biolab) and a broad-range protein ladder (NEB P7717, S1). Weights taken from Table S1 and S2 with a bin width of 569 Daltons. X-axis of histogram is reasonably aligned with the protein standard's molecular weights run alongside the SDSPAGE samples. These were compared to a histogram made using all the ribosomal protein weights taken from the Uniprot database (C, Figure 7).

The mismatch observed between the SDS-PAGE profile and the histogram of protein weights indicates that the protein is sitting slightly off from their expected molecular weight (Figure 7). The low molecular weight peaks do not align perfectly because the gel was stopped before the proteins ran off, resulting in the very small proteins being bunched at the bottom (A-C, Figure 7). It should also be noted that the larger a protein's mass, the large the signal from it. Therefore, the count does not properly indicate the strength of the signal, especially when dealing with very small proteins (about $5 \mathrm{kDa})(\mathrm{C}$, Figure 7). With these discrepancies in mind, we can see fairly good alignment of expected protein represented by the histogram and the observed protein represented by the SDS-PAGE profile. When comparing profiles of the tight-coupled ribosomes (B, Figure 7) to the ribosomes using purification done in Section 2.1.3 (A, Figure 7) there is a slight discrepancy in the peaks near $17 \mathrm{kDa}$, but it is otherwise similar. This shows that the proteins content is not 


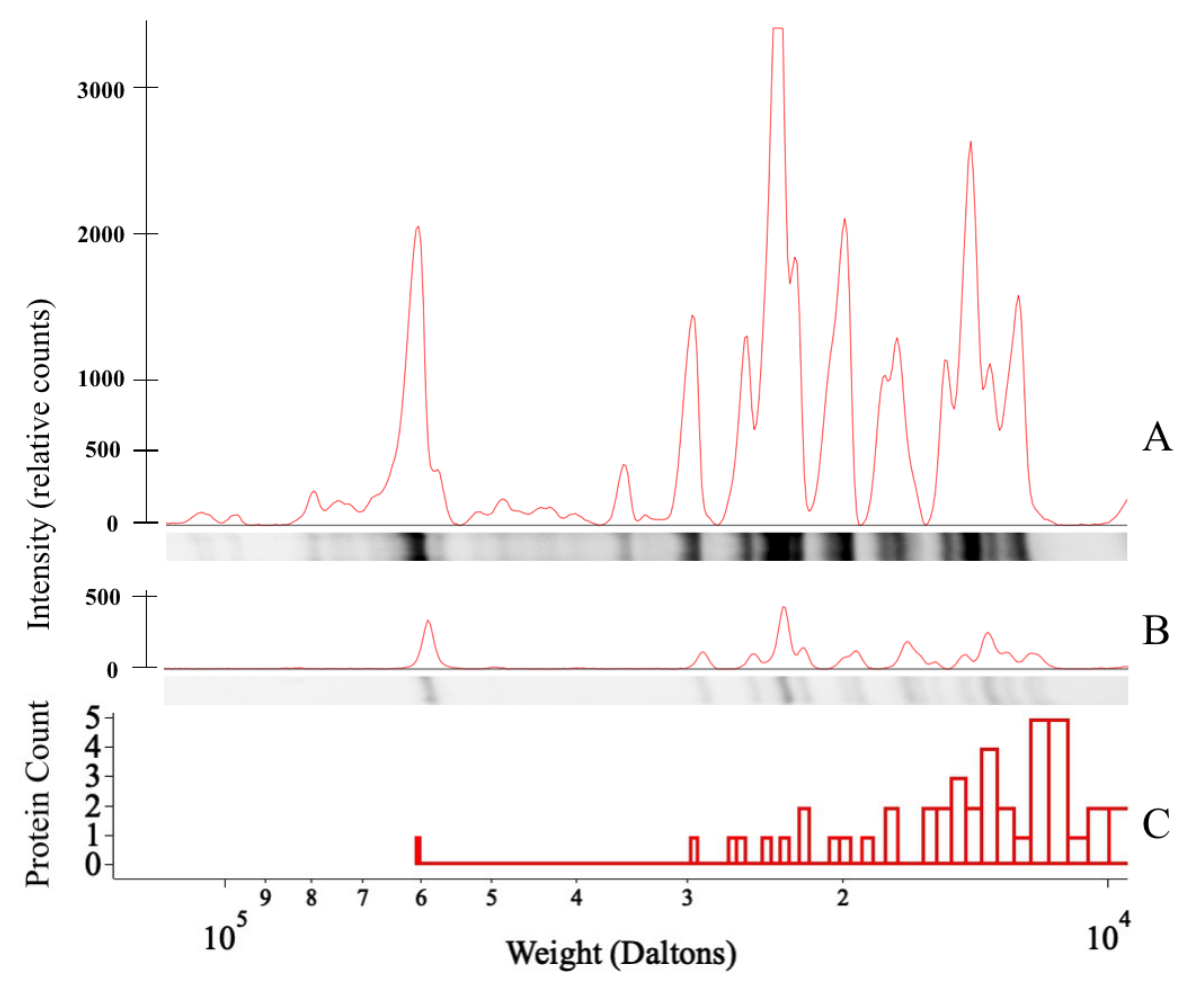

Figure 7: SDS-PAGE profiles. A: Ribosomes purified by novel method. B: Tightcouple method. C: Histogram of all ribosomal proteins. Weights taken from Table S1 and S2 with a bin width of 569 Daltons. X-axis of histogram is reasonably aligned with the protein standard's molecular weights run alongside the SDSPAGE samples.

significantly different between the novel 2.1.3 purification and tight couple ribosomal preparation.

\subsubsection{Dynamic Light Scattering}

DLS measurements are based on the principle of single light scattering, which is fulfilled when a system scatters so weakly that the probability of a photon being scattered twice is negligible. Light scattering occurs where there is a change in dielectric properties between two substances, causing a change in the refractive index. DLS measures the fluctuations in a scattering pattern as a function of time. In the experimental setup, the detector captures scattered intensities as an 
interference pattern or speckled pattern. Dispersed particles in solution perform Brownian motion, resulting in a change in the speckle pattern. In short, the fluctuations in the speckle pattern are directly related to the diffusion of molecules in a solvent [16]. The speckle pattern can be characterized by an auto-correlation function $g_{2}(\tau)$. If the molecule has a larger radius of hydration, it moves more slowly through the solution, which is reflected in $g_{2}(\tau)$. A molecule's translational diffusion coefficient, $D$, is defined as $D=\frac{k_{b} T}{6 \pi n R_{h}}$, as $K_{B}$ is the Boltzmann constant, $q$ is the wave vector, $T$ is temperature $n$ is the viscosity of the solvent, and $R_{h}$ is the hydrodynamic radius. DLS can find the $R_{h}$ from finding this translational diffusion coefficient $(D) . D$ is found from the measured intensity auto-correlation function $g_{2}(\tau)$ where $g_{2}(\tau) \leq I(t) I(t+\tau)$ and $I$ is scattered light intensity at time $t[8]$. For spherical particles, $\tau$ can be defined (Equation 8, Equation 9).

$$
\begin{gathered}
g_{2}(t)=1+\exp \left(\frac{-t}{\tau}\right) \\
\tau=1 /\left(\frac{1}{\left.2 D q^{2}\right)}\right)
\end{gathered}
$$

Perfectly monodispersed population of macromolecules never occur in solution and the correlation function corresponds to multiple particle sizes. The autocorrelation can be written as the cumulant expansion (Equation 10)

$$
\ln \left[g_{2}(\tau)-1\right] \cong-\frac{t}{\tau}+\frac{\mu_{2}}{2}\left(\frac{t}{\tau}\right)^{2}
$$

Where $\frac{\mu_{2}}{2}$ is the polydispersity index, or PDI. A PDI below 0.2 , or $20 \%$ of the sample, is not a perfectly monodispersed distribution but is accepted as one in practice [15]. 


\section{Application of Dynamic Light Scattering DLS was utilized to character-}

ize and develop the protocols in this body of work. When used to characterize fractions pumped off the sucrose gradient or a sample that was exchanged with $\mathrm{D}_{2} \mathrm{O}$ buffer, the viscosity of the solvent had to be changed accordingly in the analysis. A molecule in a viscous solvent will tumble more slowly than the same molecule is a less viscous solvent. This difference will affect the measured value of $\tau$ and impact $D$ (Equation 9). It was found that the Radius of Hydration $\left(R_{h}\right)$ of the $70 \mathrm{~S}$

E. coli ribosome that was purified using the novel method above had a hydrodynamic radius of $15 \pm 2 \mathrm{~nm}$, an example of a ribosome population is shown in Figure 8. As ribosomes are a large complex that has Brownian intra-molecular motions,

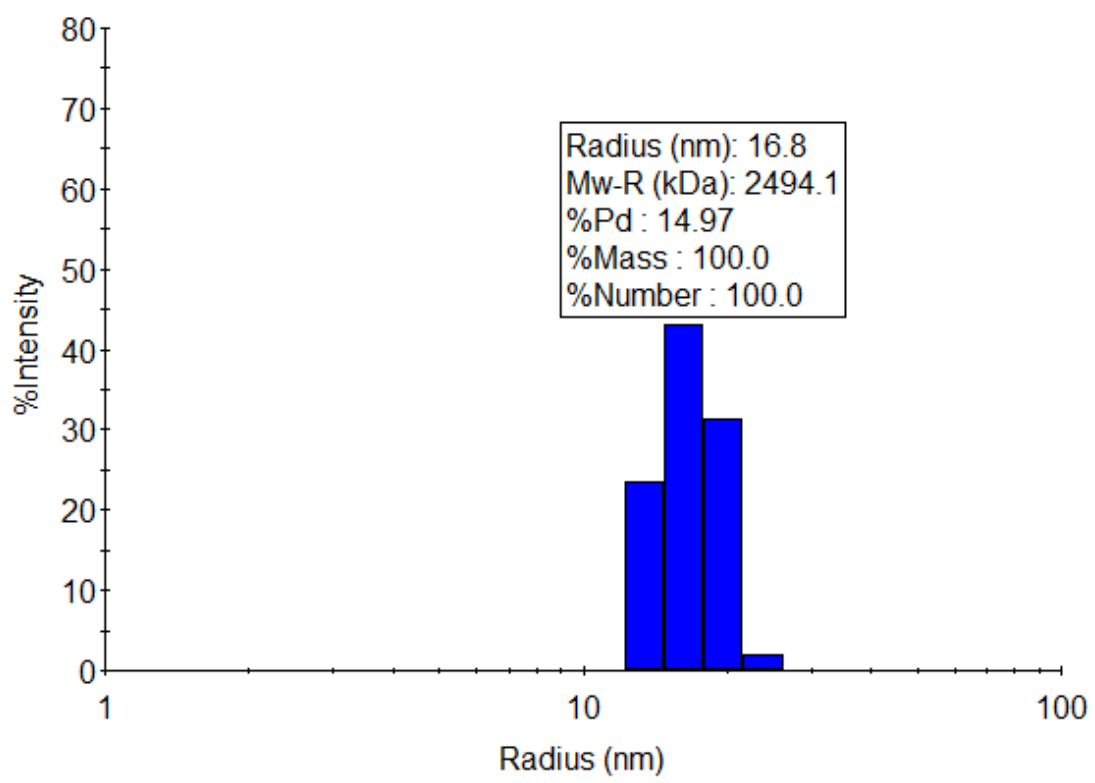

Figure 8: Ribosome population found using DLS during sample preparation optimization. Treatments included dialyzation into the correct buffer, filtration using 0.2 um syringe filter, a 5 minute heating step at $37^{\circ} \mathrm{C}$ and centrifugation with a 0.2 um filter.

it is reasonable that a small polydispersity is difficult to achieve. DLS was used to characterize each fraction pumped off the sucrose gradients that were done in the 
novel purification method in Section 2.1.3 (A-B, Figure 9).
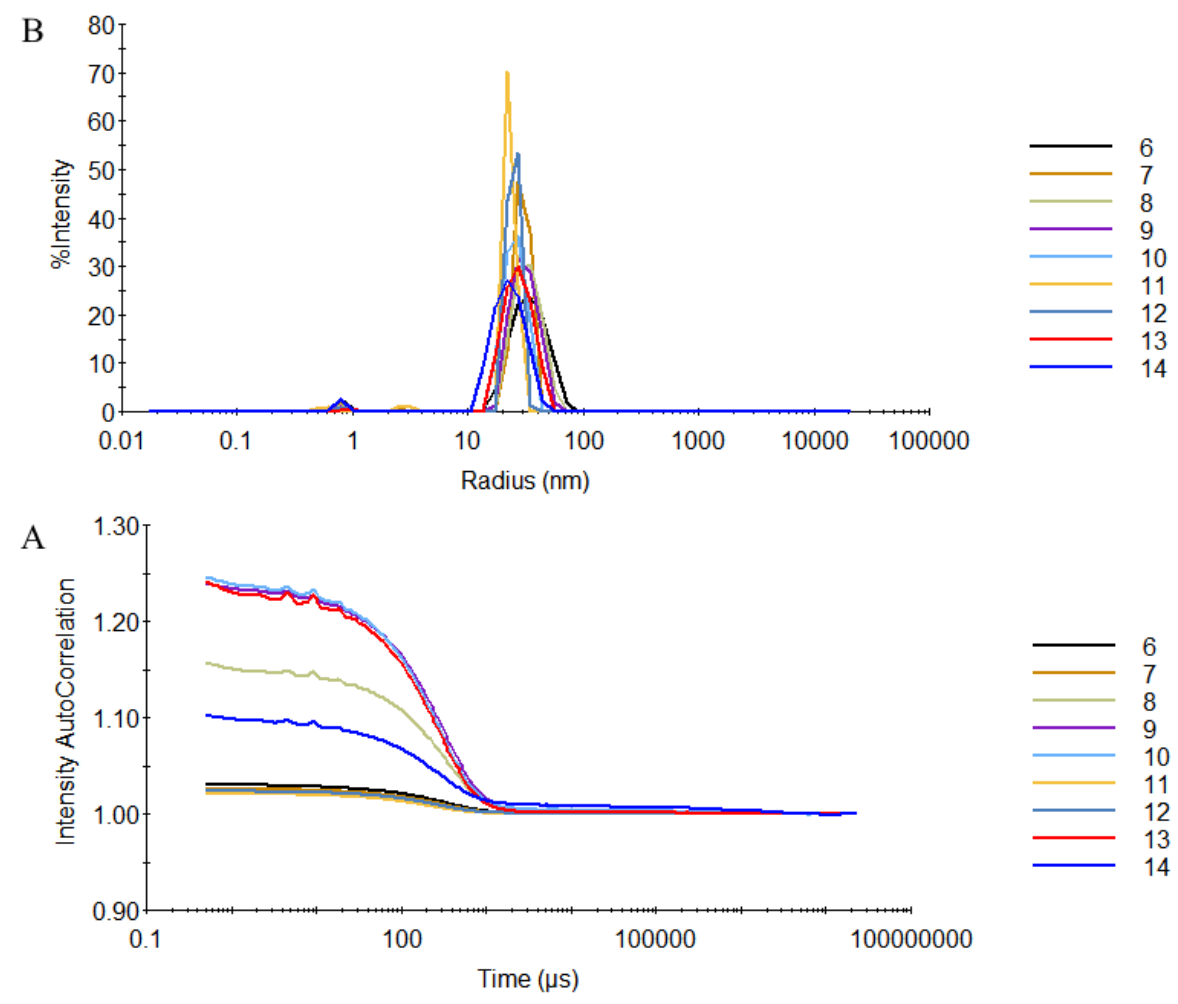

Figure 9: A: DLS Auto-correlation curves. B: Calculated populations. Numbers 1-14 represent fraction from sucrose gradient.

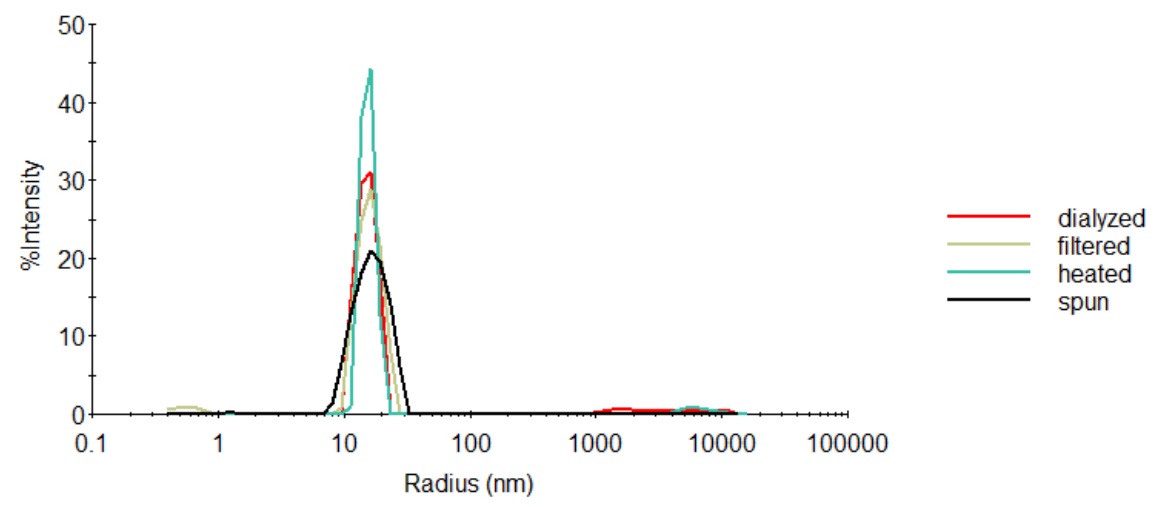

Figure 10: Populations monitored via DLS.

In particular, DLS was utilized to characterize the samples made for work done in Chapter 3, for which it is imperative to have a monodispersed population in 
solution. DLS can be used to monitor different sample treatments to determine the best method of sample preparation in a quick measurement that does not damage the sample.

Sample production optimization experiments took DLS measurements after treatments were done to purify or prepare a sample (Figure 10). When a population would appear at $1000 \mathrm{~nm}$ to $10000 \mathrm{~nm}$ radius, the syringe filter could eliminate this aggregation without causing a broadening of the polydispersity ('filtered' yellow line in Figure 10), whereas a broader population is seen to occur after a centrifugation filtering step ('spun' black line in Figure 10). Heating a sample decreases the polydispersity of the ribosome population, but small aggregations remained after heating ('heated' teal line in Figure 10). The protocol developed was to heat and then syringe filter the ribosomes before taking measurements on them. DLS was also utilized to find the highest concentration of ribosome that would not aggregate in $\mathrm{D}_{2} \mathrm{O}$ during sample preparation. Also, after samples had been exposed to a neutron beam (see Chapter 4), DLS measurements were performed to determine whether there were any changes in polydispersity.

\subsubsection{Electron Microscopy}

Electron Microscopy (EM) is a powerful method used to probe the structure of macromolecular complexes. High resolution structures, now considered to be around 3 Angstrom, are often obtained with cryo-electron microscopy (Cryo-EM) where the sample is frozen in a thin layer of ice on thin carbon-coated grids. An electron microscope can also be used to measure samples that have been prepared using a Negative Stain, where the molecules are placed directly on a carbon or copper grid at room temperature. After application of sample, uranyl formate or uranyl acetate are applied to the grid and these heavy ion solutions act as dyes that 
surround, support, and permeate the macromolecular layer. The grid is then dried and put in an electron microscope where an image is made by shining an electron beam through the sample. Due to the difference in the electron scattering density of the macromolecules and the heavy ions that surround them, the electrons are scattered by the heavy ions and not the soft matter. In this way an inverted image of the macromolecules which show up as white while their surroundings are dark. The relative contrast allows for Negative Stain to be used easily image biological samples. Negative Stain is a robust technique to find a low resolution 3D structure of a molecule but can also be applied to sample characterization to investigate macromolecular complex formation or sample homogeneity or quality [4, 20]. Often Negative Stain is used to screen samples before they are concentrated by about 10 times and made into Cryo-EM samples.

When analyzing both Negative Stained and Cryo single-molecule EM samples, the captured 2 dimensional EM images can be used to reconstruct 3-dimensional models of the macromolecule. Two main programs that do this are named RELION (REgularised LIkelihood OptimisatioN) and SPIDER (System for Processing Image Data from Electron microscopy and Related fields). The computing program RELION uses a Bayesian framework that incorporates prior knowledge and learned parameters from the data to create models [21]. The program SPIDER has implemented features such as a point spread function, which specifically is used to blurring of images around the periphery, and a spherical deconvolution, which when applied together these tools discriminate noise in the data and structural information of a molecules periphery [14]. The input for model creation programs are EM snapshots of a single molecule, which we will call "particles", the single-molecule "particles" are extracted from an EM image, called a micrograph. Initially, the user must verify the sample in the EM micrograph is good and that 
the particles selected from the micrograph are not an aggregation or an impurity. In applying SPIDER and RELION impurities can sometimes be misidentified as particles at this stage. However, these particles can be discarded during 2D classification. In this step, particles are classified on how similar the images are to one another in a computationally heavy 2D image sorting task. Functionally, this means that snapshots of a certain orientation of a molecule will be sorted into the same class. 2D classes are output and the user will manually choose the classes that portray their molecule of interest. These $2 \mathrm{D}$ classes are used to construct a 3-dimensional model with 6685 particles (Figure 11).

Both Negative Staining and Cryo-EM can probe a sample at the single-molecule level and have been heavily utilized to investigate the ribosome. In the 1980s electron microscopy was the first tool to reveal the shape of the ribosome. It elucidated large structural features such as the central protuberance, small subunit head, and other global structural aspects [10, 12, 25]. It also offered insight into the exiting nascent protein chain [29, 3]. By 1995 two main structures of the ribosomes from EM in vitreous ice had been determined [10, 25] at this point SANS was used to identify which of these EM structures was most accurate. SANS could provide a similar level of structural resolution as Cryo-EM. In the late 90s, both techniques gave structural resolution of about $2 \mathrm{~nm}$ and information from both techniques were compared [26, 27].

Application of Electron Microscopy Negative Stain was done to characterize ribosomes purified in the novel method discussed in Section 2.1.2. Specifically, EM was used to determine the amount of $50 \mathrm{~S}$ free-floating subunit in the sample. In this study, all the particles were manually selected. This step was necessary due to issues with RELION in analyzing Negative Stain micrographs be- 


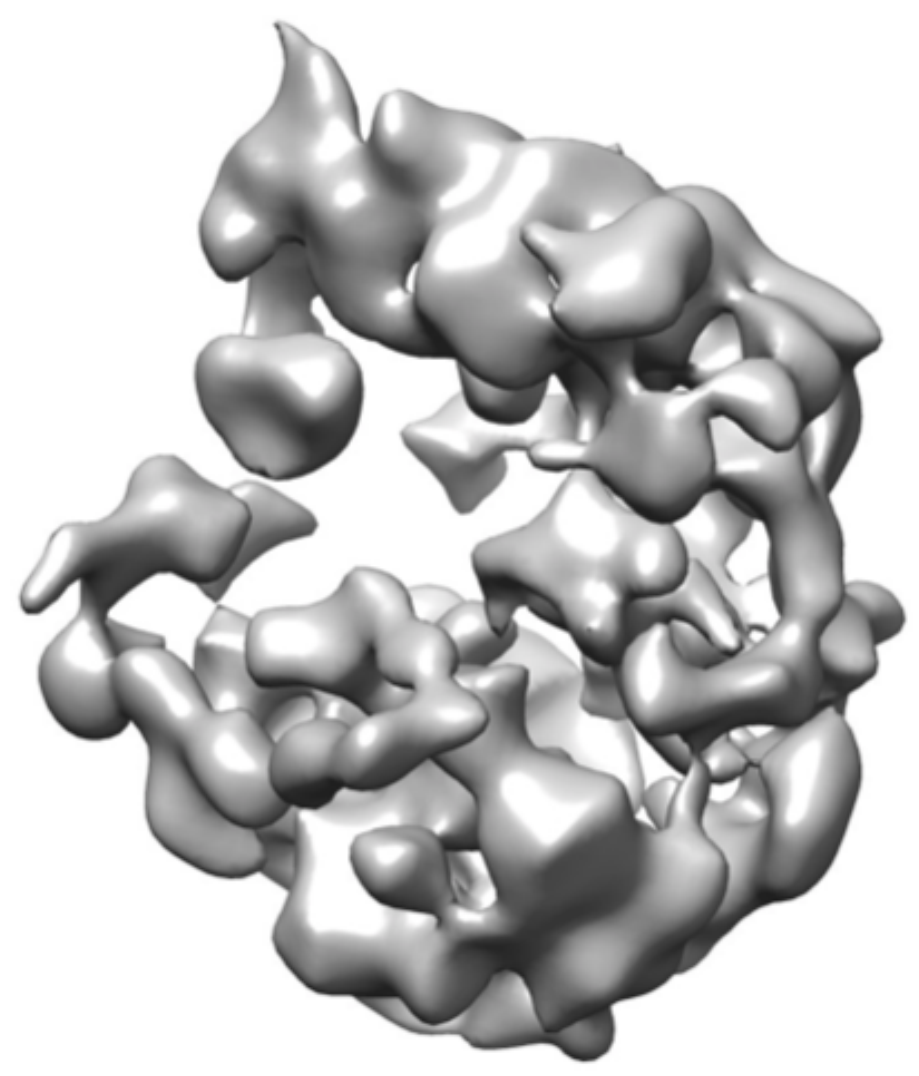

Figure 11: 3D model of the 70S E. coli ribosome made using the software RELION.

cause the software is optimized for Cryo-EM images. Particle selection occurred in two rounds. The first round was aimed at counting the number of 70S in the micrographs and the second round was aimed at determining the number of free $50 \mathrm{~S}$ subunits. The $70 \mathrm{~S}$ particles were identified by their size and by having a visible cavity. These particles were sorted into 2D classes, which were then used to construct a 3D model of the $70 \mathrm{~S}$ ribosome at $2.1 \mathrm{~nm}$ resolution. This structure was confirmed by comparison to known 70S structures (Figure S2). In a second round of manual particle picking was done and care was taken to only pick small particles. These particles were then sorted into 2D classes.

Quantifying the ratio of $50 \mathrm{~S}$ to $70 \mathrm{~S}$ is important for SAS work, as described in 

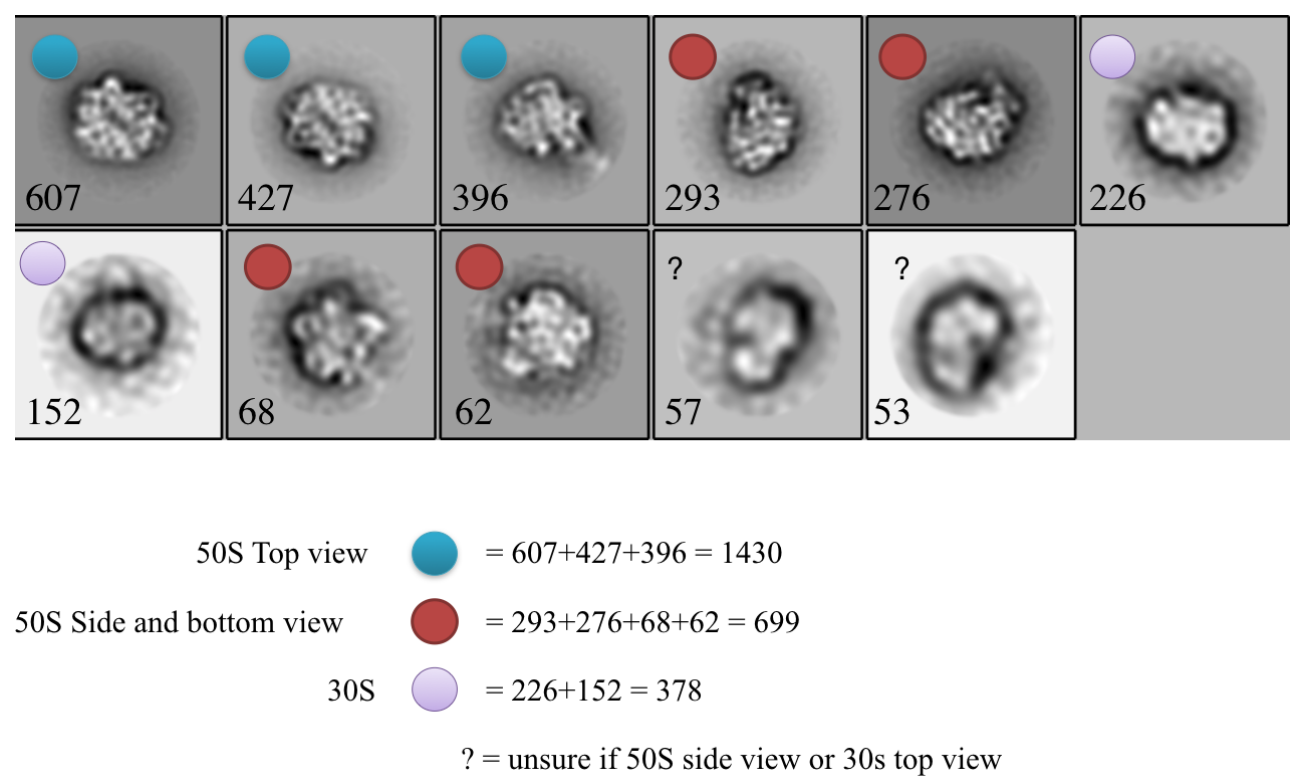

Figure 12: Negative Stain 2D classification of the manually picked small particles. Each class shows a representative and amount of the particles within the class.

Section 1.4. The amount of free $50 \mathrm{~S}$ was calculated assuming that the amount of $30 S$ found in the Negative Stain micrographs would be bound to a 50 S partner in solution. In this technique, there is an uncertainty of whether a 50S that is seen from a top view has a $30 \mathrm{~S}$ bound beneath it. An upper bound of free $50 \mathrm{~S}$ was calculated that assumed the top views of the $50 \mathrm{~S}$ do not have $30 \mathrm{~S}$ bound beneath them and a lower bound was calculated that assumed all $50 \mathrm{~S}$ top views had a $30 \mathrm{~S}$ bound below them, calculations in Equations 11 and 12, respectively. The amount of free $50 \mathrm{~S}$ in solution is between $20.3 \%$ and $3.6 \%$, using inputs taken from particle counts found in Figure 12 .

$$
\begin{aligned}
& \frac{1430+699-378}{1430+699+6685}=0.203 \\
& \frac{699-378}{1430+699+6685}=0.036
\end{aligned}
$$




\section{References}

[1] D E Agafonov, V A Kolb, and A S Spirin. "Ribosome-associated protein that inhibits translation at the aminoacyl-tRNA binding stage." eng. In: EMBO reports 2.5 (May 2001), pp. 399-402. ISSN: 1469-221X (Print). DOI: 10.1093/ embo-reports/kve091.

[2] Dmitry E Agafonov, Vyacheslav A Kolb, and Alexander S Spirin. "Proteins on ribosome surface: Measurements of protein exposure by hot tritium bombardmenttechnique". In: Proceedings of the National Academy of Sciences 94.24 (Nov. 1997), 12892 LP -12897. DOI: 10.1073/pnas .94 .24 .12892. URL: http: //www . pnas . org/content/94/24/12892. abstract.

[3] C Bernabeu and J A Lake. "Nascent polypeptide chains emerge from the exit domain of the large ribosomal subunit: immune mapping of the nascent chain." eng. In: Proceedings of the National Academy of Sciences of the United States of America 79.10 (May 1982), pp. 3111-3115. ISSN: 0027-8424 (Print). DOI: $10.1073 /$ pnas.79.10.3111.

[4] David S. Booth, Agustin Avila-Sakar, and Yifan Cheng. "Visualizing Proteins and Macromolecular Complexes by Negative Stain EM: from Grid Preparation to Image Acquisition". In: Journal of Visualized Experiments 58 (2011), pp. 1-7. DOI: $10.3791 / 3227$.

[5] Johan Busselez et al. "Cryo-Electron Tomography and Proteomics studies of centrosomes from differentiated quiescent thymocytes". In: Scientific Reports 9.1 (2019), p. 7187. ISSN: 2045-2322. DOI: 10.1038/s41598-019-43338-9. URL: https://doi.org/10.1038/s41598-019-43338-9.

[6] Ed T Buurman et al. "Novel Rapidly Diversifiable Antimicrobial RNA Polymerase Switch Region Inhibitors with Confirmed Mode of Action in ispan 
class="named-content genus-species" id="named-content-1" $¿$ Haemophilus influenzaej/span i". In: Journal of Bacteriology 194.20 (Oct. 2012), 5504 LP 5512. DOI: 10.1128/JB.01103-12. URL: http://jb.asm.org/content/194/ 20/5504.abstract.

[7] Konstantin Byrgazov et al. "Structural basis for the interaction of protein S1 with the Escherichia coli ribosome". eng. In: Nucleic acids research 43.1 (Jan. 2015), pp. 661-673. ISSN: 1362-4962. DOI: 10.1093/nar/gku1314. URL: https: //www .ncbi.nlm.nih.gov/pubmed/25510494\%20https ://www .ncbi.nlm. nih.gov/pmc/articles/PMC4288201/

[8] Dewey K Carpenter. "Dynamic Light Scattering with Applications to Chemistry, Biology, and Physics (Berne, Bruce J.; Pecora, Robert)". In: Journal of Chemical Education 54.10 (Oct. 1977), A430. ISSN: 0021-9584. DOI: 10.1021/ ed054pA430.1. URL: https://doi.org/10.1021/ed054pA430.1.

[9] Josefine Ederth et al. "A single-step method for purification of active Histagged ribosomes from a genetically engineered Escherichia coli". In: $\mathrm{Nu}$ cleic Acids Res 37.2 (Feb. 2009), e15-e15. ISSN: 0305-1048. DOI: 10.1093/nar/ gkn992. URL: http://www.ncbi.nlm.nih.gov/pmc/articles/PMC2632923//

[10] Joachim Frank et al. "A model of protein synthesis based on cryo-electron microscopy of the E. coli ribosome". In: Nature 376.6539 (1995), pp. 441-444. ISSN: 1476-4687. DOI: 10.1038/376441a0, URL: https://doi .org/10.1038/ 376441a0,

[11] Robyn Hickerson et al. "Measurement of Internal Movements within the $30 \mathrm{~S}$ Ribosomal Subunit Using Förster Resonance Energy Transfer". In: Journal of Molecular Biology 354.2 (2005), pp. 459-472. ISSN: 0022-2836. DOI: https : // 
doi.org/10.1016/j.jmb.2005.09.010. URL: http://www. sciencedirect. com/science/article/pii/S0022283605010661

[12] W Hoppe, H Oettl, and H R Tietz. “Negatively stained 50 S ribosomal subunits of Escherichia coli." eng. In: Journal of molecular biology 192.2 (Nov. 1986), pp. 291-322. ISSN: 0022-2836 (Print).

[13] Pierre C Jelenc. "Rapid purification of highly active ribosomes from Escherichia coli". In: Analytical Biochemistry 105.1 (1980), pp. 369-374. ISSN: 0003-2697. DOI: https : / / doi . org / 10 . 1016 / 0003 - 2697 (80 ) 90472-8. URL: http : //www.sciencedirect.com/science/article/pii/0003269780904728.

[14] Gregory P Kishchenko and Ardean Leith. "Spherical deconvolution improves quality of single particle reconstruction". In: Journal of Structural Biology 187.1 (2014), pp. 84-92. ISSN: 1047-8477. DOI: https : //doi .org/10 .1016/j · jsb. 2014.05.002, URL: http://wWW . sciencedirect . com/science/article/ pii/S1047847714001087.

[15] D Langevin et al. "Inter-laboratory comparison of nanoparticle size measurements using dynamic light scattering and differential centrifugal sedimentation". In: NanoImpact 10 (2018), pp. 97-107. ISSN: 2452-0748. DOI: https : // doi.org/10.1016/j.impact.2017.12.004. URL: http://wWw.sciencedirect. com/science/article/pii/S2452074817300903

[16] Ye Li, Vassiliy Lubchenko, and Peter G Vekilov. “The use of dynamic light scattering and Brownian microscopy to characterize protein aggregation". In: Review of Scientific Instruments 82.5 (May 2011), p. 53106. ISSN: 0034-6748. DOI: 10.1063/1.3592581. URL: https://doi.org/10.1063/1.3592581.

[17] Sameh Magdeldin et al. "Basics and recent advances of two dimensionalpolyacrylamide gel electrophoresis". eng. In: Clinical proteomics 11.1 (Apr. 
2014), p. 16. ISSN: 1542-6416. DOI: 10.1186/1559-0275-11-16. URL: https : //www.ncbi.nlm.nih.gov/pubmed/24735559\%20https ://www .ncbi.nlm . nih.gov/pmc/articles/PMC3996944/

[18] Peter B Moore. "Ribosomes and Protein Synthesis: A Practical Approach. G. Spedding ". In: The Quarterly Review of Biology 67.2 (June 1992), p. 198. ISSN: 0033-5770. DOI:10.1086/417568, URL: https://doi.org/10.1086/417568.

[19] Thierry Rabilloud and Cécile Lelong. "Two-dimensional gel electrophoresis in proteomics: A tutorial". In: Journal of Proteomics 74.10 (2011), pp. 18291841. ISSN: 1874-3919. DOI: https : //doi .org/10 . 1016/j . jprot . 2011. 05. 040. URL: http://www . sciencedirect .com/science/article/pii/ S1874391911002545.

[20] Charlotte A. Scarff et al. "Variations on Negative Stain Electron Microscopy Methods: Tools for Tackling Challenging Systems". In: Journal of Visualized Experiments 132 (2018), pp. 1-8. DOI: 10.3791/57199.

[21] Sjors H W Scheres. "A Bayesian View on Cryo-EM Structure Determination". In: Journal of Molecular Biology 415.2 (2012), pp. 406-418. ISSN: 0022-2836. DOI: https : //doi .org/10.1016/j.jmb . 2011 .11 .010, URL: http://www . sciencedirect.com/science/article/pii/S0022283611012290.

[22] Bassem Shebl et al. "Preparation of ribosomes for smFRET studies: A simplified approach". In: Archives of Biochemistry and Biophysics 603 (2016), pp. 118130. ISSN: 0003-9861. DOI: http : // dx . doi . org/10 . 1016/ j . abb . 2016 . 05.010. URL: http://www . sciencedirect.com/science/article/pii/ S0003986116301576. 
[23] Y Shimizu et al. "Cell-free translation reconstituted with purified components." eng. In: Nature biotechnology 19.8 (Aug. 2001), pp. 751-755. ISSN: 10870156 (Print). DOI: 10.1038/90802.

[24] Theophil Staehelin and Donna R B T - Methods in Enzymology Maglott. “[47] Preparation of Escherchia coli ribosomal subunits active in polypeptide synthesis". In: Part C: Nucleic Acids and Protein Synthesis. Vol. 20. Academic Press, 1971, pp. 449-456. ISBN: 0076-6879. DOI: https ://doi .org/10 .1016/ S0076-6879(71) 20049-5. URL: http://www . sciencedirect.com/science/ article/pii/S0076687971200495.

[25] Holger Stark et al. "The 70S Escherichia coli ribosome at 23 å resolution: fitting the ribosomal RNA". In: Structure 3.8 (1995), pp. 815-821. ISSN: 09692126. DOI: https://doi.org/10.1016/S0969-2126(01)00216-7. URL: http: //www.sciencedirect.com/science/article/pii/S0969212601002167.

[26] D I Svergun et al. "Solution scattering structural analysis of the $70 \mathrm{~S}$ Escherichia coli ribosome by contrast variation. I. invariants and validation of electron microscopy models11Edited by M. F. Moody". In: Journal of Molecular Biology 271.4 (1997), pp. 588-601. ISSN: 0022-2836. DOI: https ://doi.org/ 10.1006/jmbi.1997.1190. URL: http://www.sciencedirect.com/science/ article/pii/S0022283697911906.

[27] D I Svergun et al. "Solution scattering structural analysis of the $70 \mathrm{~s}$ Escherichia coli ribosome by contrast variation. IIt. A model of the ribosome and its RNA at $3.5 \mathrm{~nm}$ resolution1+Paper I in this series is the accompanying paper, Svergun et al. (1997)1Edited by M. F. Moody". In: Journal of Molecular Biology 271.4 (1997), pp. 602-618. ISSN: 0022-2836. DOI: https : // doi . org/ 
10.1006/jmbi.1997.1191. URL: http://www.sciencedirect.com/science/ article/pii/S0022283697911918.

[28] Törnkvist, M., Larsson, G. Enfors. “Protein release and foaming in Escherichia coli cultures grown in minimal medium". In: Bioprocess Engineering 15.231 (1996). DOI: https://doi.org/10.1007/BF02391583.

[29] A Yonath, K R Leonard, and H G Wittmann. "A tunnel in the large ribosomal subunit revealed by three-dimensional image reconstruction". In: Science 236.4803 (May 1987), 813 LP -816. DOI: 10.1126/science.3576200. URL: http://science.sciencemag.org/content/236/4803/813.abstract.

[30] Elaine M Youngman and Rachel Green. "Affinity purification of in vivoassembled ribosomes for in vitro biochemical analysis". In: Methods 36.3 (2005), pp. 305-312. ISSN: 1046-2023. DOI: https : //doi .org/10 . 1016/j · ymeth . 2005.04.007. URL: http : //wWW . sciencedirect .com/science/article/ pii/S1046202305000903. 


\section{Structural Performance of Ribosomal Proteins during mRNA Un- winding by E. coli Ribosomes in Solution}

This chapter and the supplementary figures referenced within are converted from a manuscript to be submitted October 2019.

Emily Armbruster ${ }^{1,2}$, Kevin Weiss ${ }^{2}$, Loukas Petridis ${ }^{2}$, Jose Borreguero Calvo ${ }^{2}$, James Byrnes $^{3}$, Peter Cornish ${ }^{1}$, Sai Venkatesh Pingali ${ }^{2}$

${ }^{1}$ Department of Biochemistry, University of Missouri, Columbia, MO

${ }^{2}$ Oak Ridge National Laboratory, Oak Ridge, TN

${ }^{3}$ Brookhaven National Laboratory, Upton, NY

\subsection{Summary}

Proteins, the workhorse of a biological cell, are produced and distributed by a large, flexible, macromolecular machine called the ribosome. Messenger RNA (mRNA) is translated by the ribosome to create a protein in a process that involves molecular movements of the ribosome and determining the various structural conformations involved can provide insight toward understanding ribosomal function. Currently, the field of functionally oriented structural biology emphasizes the importance of mapping conformational space. However, mapping the continuous structural variation of the ribosome is difficult due to low energetic barriers between ribosomal conformational states [8,9]. This difficulty has interfered in characterizing the conformational distortions of the ribosome as it unwinds structured mRNA during translation, an important phenomenon that impacts the production and diversity of proteome. This study uses experimental small-angle scattering methods to divulge the structural dimensions of $70 \mathrm{~S}$ E. coli ribosome in solution. We observed structural differences between a ribosome that is bound to structured mRNA and a ribosome that is vacant or bound to linear mRNA. This comparison shows the proteins on a ribosome interacting with an mRNA secondary structure 
elongates $27 \%$ in the maximum dimension of ribosomal proteins.

\subsection{Introduction}

The ribosome is a macromolecular complex that serves as the platform for the production and distribution of proteins in a cell. The E. coli $70 \mathrm{~S}$ bacterial ribosome is made up of three large RNA components called the 5S, 16S, and 23S rRNA molecules and 54 small ribosomal proteins bound to these rRNAs around the periphery of the complex. A nascent peptide is made as amino acid building blocks are shuttled by transfer RNA (tRNA) molecules into and through the ribosome. tRNA positioning is critical to the synthesis mechanism. Binding and movements of tRNA are dictated by interactions with the ribosomal components, protein cofactors, and the messenger RNA (mRNA). An mRNA molecule acts as a template to be read by the ribosome and dictates the order of tRNA cycling through the ribosome. During protein elongation, the mRNA must be linear to move through the ribosome, though sometimes the mRNA molecule in a cell can fold into secondary structures such as hairpins or pseudoknots. When an mRNA secondary structure is at a ribosome's mRNA entrance tunnel, the ribosome applies a helicase mechanism. An active helicase mechanism can result in slippage of the ribosome on the mRNA, a phenomena called frameshifting, which can lead to premature termination of the protein being synthesized or a different protein being produced. Structured mRNA that has defined frameshifting efficiency is called programmed frameshifting [21]. Programmed frameshifting allows the genome to contain more information that what is linearly encoded, as one mRNA can be translated to produce more than one protein [13, 25]. The fidelity of translation can drop from its usual $99.9 \%$ to around $50 \%$ during programmed frameshifting [2], and rates of translocation movements are 10-fold slower than observed in normal mode trans- 
lation [3, 5].

As the ribosome happens upon a structure in the mRNA strand it pauses [5] and gets stuck in a conformational state [18]. The conformational state that the ribosome occupies during these mRNA unwinding events is not well understood. The ribosome may occupy a hyper-rotated state where subunit rotation increases to $22^{\circ}$, which is substantially further than the rotated/hybrid state that has a rotation of $4-10^{\circ}$ from the classical non-rotated conformational state [18]. Theoretical studies propose that as the ribosome attempts to unwind a structured mRNA, the small subunit rolls about the h44 helix of the $16 \mathrm{~S}$ rRNA by around $12.7^{\circ}$ [4]. The conformational state of the ribosome during mRNA unwinding remains uncertain. We applied small angle neutron/X-ray scattering to find global structural information of a ribosome unwinding mRNA with a secondary structure and compare this structural information to a vacant ribosome as well as a ribosome bound to linear mRNA.

Small Angle Scattering (SAS) is a method similar to X-ray crystallography in which an incident beam gets scattered by a sample and the interference pattern from the scattered beam is captured on a camera. The main difference between X-ray crystallography and small angle scattering is that the scattering molecule is tumbling and flexible in solution. The data collected is an ensemble average of the scattering from a molecule in all orientations over time, which means that the 2D scattering pattern taken by the camera shows isotropic scattering and therefore can be simplified into a 1D intensity curve. These curves contain information that delineate all intra-molecular distances and yield information about the largest dimensions of a molecule. Specifically, the radius of gyration and maximum diameter is found, as well as information about a macromolecule's shape. In this study, we find dimensional and shape changes of the ribosome as it unwinds mRNA and 
compare it to the dimensions and shape of vacant ribosomes and ribosomes that are bound to linear mRNA.
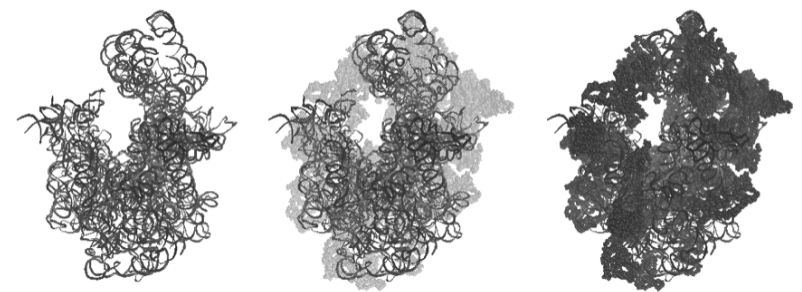

Figure 13: Schematic depicts the scattering contribution of the protein and rRNA. From left to right: SANS $42 \% \mathrm{D}_{2} \mathrm{O}$, SAXS, SANS $100 \% \mathrm{D}_{2} \mathrm{O}$

The SAS technique observes the global structure of a macromolecule in solution and can provide valuable information about a biological molecule's flexibility and water interaction. The ribosome is known to function as a Brownian ratchet with large conformational changes that occur spontaneously in solution using single molecule Föster Resonant Energy Transfer [8, 9]. Although, smFRET measurements report on only two or three coordinates of a system and may not report on optimal coordinates to understand ribosomal dynamics [15]. This study suggests that care must be taken in the placement of fluorophores and analysis of smFRET measurements, as SAS measurements show large extensions, flexibility, and solvent interactions of ribosomal proteins. Specifically, the studies here indicate the ribosomal proteins are more flexible and interact with the solvent to a greater degree than is often observed by structural studies. These results are applicable to studies that combine Cryo-EM, smFRET, and MD methods. 


\subsection{Results}

\subsubsection{Small-Angle Scattering observes intermolecular dimensions of the rRNA and the ribosomal proteins}

This study uses the natural scattering length density of protein and RNA to provide three different perspectives that observe the dimensions of the E. coli $70 \mathrm{~S}$ scattering particle as the amount of protein scattering contribution increases from non-existent to domination of the scattering signal (Figure 13). The SANS measurement in a buffer with $42 \% \mathrm{D}_{2} \mathrm{O}$ gives the perspective of the rRNA structure only and shows the rRNA from all three samples have a radius of gyration $\left(R_{g}\right)$ of $88 \pm 1 \AA$ and a maximum dimension of $236 \AA$ (Figure 14 top panel, Table 1). The SAXS measurement (Figure 14 middle panel, Table 1) and the SANS measurement in a $100 \% \mathrm{D}_{2} \mathrm{O}$ buffer (Figure 14 bottom panel, Table 1) perceives both the rRNA and the ribosomal proteins, but with different relative RNA/protein contrasts. The particle seen by SAXS has an $\mathrm{R}_{g}$ about $30 \AA$ smaller than that seen in $100 \% \mathrm{D}_{2} \mathrm{O}$ buffer by SANS. The SAXS $\mathrm{R}_{g}$ is much closer to the size of the rRNA particle seen in $42 \% \mathrm{D}_{2} \mathrm{O}$ buffer by SANS. Proteins and RNA have similar contrast with water in SAXS, but most ribosomal proteins have a small size, and many of them extend out into the solvent. It is reasonable that the protein signal can be lost within the strong RNA scattering signal in SAXS due to protein size and moderate contrast. The $100 \% \mathrm{D}_{2} \mathrm{O}$ buffer SANS measurement picks up the most massive scattering particles observed, which is expected because ribosomal proteins add a significant increase to the external dimensions of the molecule (Figure 13). We have successfully used these three measurements to tune the focus of our view of the components of the 70S E. coli ribosome. These SAS measurements have not only allowed for the observation of the ribosome in a novel state as it interacts 
with structured mRNA, but also provide important information that reports on the flexibility of the rRNA and ribosomal proteins.
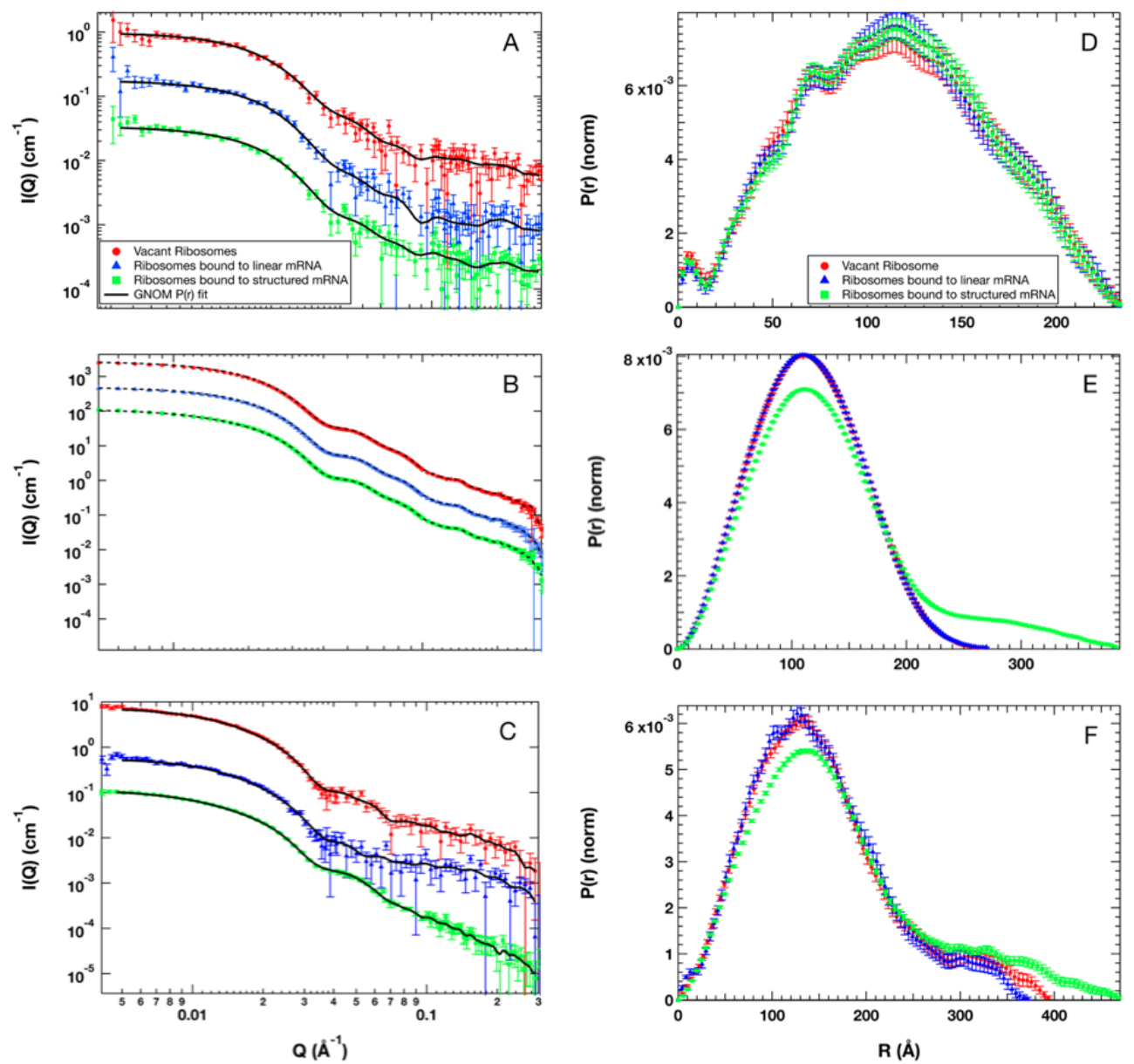

Figure 14: Scattering intensities and GNOM P(r) fit (left) and distance distribution curve plot (right). Top: SANS measurements and $42 \% \mathrm{D}_{2} \mathrm{O}$. Middle: SAXS measurements. Bottom: SANS measurements at $100 \% \mathrm{D}_{2} \mathrm{O}$. Scaled for visual clarity.

\subsubsection{Solution Scattering is from the Flexible Ribosome in Solution}

Previously, Small Angle Neutron Scattering (SANS) was used to find the solution structure of a vacant 70S E. coli ribosome [23]. The rRNA and ribosomal protein dimensions found in this study had an $\mathrm{R}_{g}$ of $82 \pm 2 \AA$ and $105 \pm 5 \AA$, respectively. These reported values are smaller than our results of $88 \pm 1 \AA$ for rRNA scattering 
Table 1: Distance measurements found by Guinier analysis and GNOM P(r) fitting.

\begin{tabular}{|c|c|c|c|c|}
\hline \multirow{2}{*}{ Samples } & \multirow{2}{*}{$\begin{array}{l}\mathbf{Q}_{\min } \\
\left(\AA^{-1}\right)\end{array}$} & \multirow{2}{*}{$\begin{array}{c}\text { Guinier } \\
\left.\boldsymbol{R}_{\mathbf{g}}(\AA)\right)\end{array}$} & \multicolumn{2}{|c|}{ GNOM } \\
\hline & & & $R_{\mathrm{g}}(\AA)$ & $D_{\max }(\AA)$ \\
\hline \multicolumn{5}{|c|}{ SANS in $42 \% \mathrm{D}_{2} \mathrm{O}$ buffer; RNA only } \\
\hline Vacant $^{\star \star \star}$ & 0.0044 & $80 \pm 7$ & $88 \pm 1$ & 236 \\
\hline Linear mRNA ${ }^{\star \star}$ & 0.0044 & $91 \pm 7$ & $88 \pm 1$ & 236 \\
\hline Structured mRNA* & 0.0044 & $94 \pm 7$ & $88 \pm 1$ & 236 \\
\hline \multicolumn{5}{|c|}{ SAXS in $0 \% D_{2} O$ buffer; RNA weighted } \\
\hline Vacant $^{\star \star \star}$ & 0.005 & $90.4 \pm 0.4$ & $87.5 \pm 0.1$ & 269 \\
\hline Linear mRNA ${ }^{\star \star}$ & 0.005 & $91.3 \pm 0.4$ & $87.6 \pm 0.1$ & 271 \\
\hline Structured mRNA* & 0.005 & $102.2 \pm 1.4$ & $105 \pm 2$ & 385 \\
\hline \multicolumn{5}{|c|}{ SANS in $100 \% D_{2} O$ buffer; Protein weighted } \\
\hline Vacant $^{\star \star \star}$ & 0.005 & $121 \pm 4$ & $120 \pm 2$ & 398 \\
\hline Linear mRNA ${ }^{\star \star}$ & 0.005 & $122 \pm 8$ & $116 \pm 2$ & 376 \\
\hline Structured mRNA* & 0.005 & $133 \pm 3$ & $135 \pm 2$ & 475 \\
\hline
\end{tabular}

and $120 \pm 2 \AA$ from protein weighted scattering of a vacant ribosome. It is reasonable that the difference in the dimensions reported in this study and previous studies is due to the difference in sample creation. The ribosome samples from previous studies were created by mixing isolated rRNA and total protein, whereas the ribosomes in this study are assembled in vivo. Ribosomes created by mixing isolated rRNA and total protein show up to $50 \%$ less activity than ribosomes assembled in vivo [11]. Thus, the larger $\mathrm{R}_{g}$ of $6 \pm 3 \AA$ in the rRNA component is likely due to the differences in rRNA folding and flexibility. Similarly, the peripheral protein extensions of $15 \pm 7 \AA$ is likely due to a lower quantity of ribosomal proteins in the particle or a difference in ribosomal protein interactions with the rRNA and water.

The choice of buffer plays a critical role in the state of the ribosome during measurement. The polyamine buffer used in our measurements include $6 \mathrm{mM} \mathrm{MgCL}_{2}$, $2 \mathrm{mM}$ Spermidine, and $0.1 \mathrm{mM}$ Spermine that keep the ribosome subunits together, yet flexible [24]. This buffer is often used in smFRET and dynamics experiments 
[18]. On the other hand, most structural studies use $10 \mathrm{mM} \mathrm{MgCL}_{2}$ [23], which ensures subunit association but decreases the flexibility of the ribosome [28, 29]. To test the influence on buffer components on the ribosome, SAXS measurements were taken of ribosomes in polyamine buffer (20 mM HEPES pH 7.5, $6 \mathrm{mM} \mathrm{MgCl}_{2}$, $150 \mathrm{mM} \mathrm{NH}_{4} \mathrm{Cl}, 6 \mathrm{mM} \beta \mathrm{ME}, 2 \mathrm{mM}$ Spermidine, $0.1 \mathrm{mM}$ Spermine) and buffer containing $10 \mathrm{mM} \mathrm{MgCl}_{2}$ (100 mM Imidazol pH 7.5, $10 \mathrm{mM} \mathrm{MgCl}$, $100 \mathrm{mM} \mathrm{KCl}$ [23]). The Guinier analysis of these two samples shows no significant change between the maximum dimensions of the molecule. The $R_{g}$ of the ribosomes in polyamine buffer is $92 \pm 2$. The same ribosomes in a buffer containing $10 \mathrm{mM} \mathrm{MgCl}_{2}$ is $90 \pm$ 2. Thus, any structural change due to the buffer components is undetectable using SAS.

The measurements by SAXS and SANS in $100 \% \mathrm{D}_{2} \mathrm{O}$ buffer show the ribosome bound to structured mRNA is more elongated than either the vacant ribosome or a ribosome bound to linear mRNA. In $100 \% \mathrm{D}_{2} \mathrm{O}$ buffer measurements the maximum dimension increases by $77 \AA$ and $99 \AA$ while $\mathrm{R}_{g}$ increases by $15 \AA$ and 19 $\AA$, respectively (Figure 14 when the ribosome is bound to an mRNA that has a hairpin in the +12 position. An extended Guinier analysis of the scattering intensity of the SANS data in $100 \% \mathrm{D}_{2} \mathrm{O}$ buffer reports that the larger particle size is not due to aggregation (Table S4). The conformational extension of the ribosome bound to structured mRNA appears to be an extension of the ribosomal proteins without a change in rRNA dimensions. SAXS scattering of the three samples have the same shape in the high $\mathrm{Q}$ range $(0.02$ to $0.3 \AA$ - -1$)$ and the sample that contained ribosomes bound to structured mRNA only deviates in the low $Q$ region (0.005 to $0.02 \AA$-1)(Figure 14 middle panel, Figure S3). The shape of the SAS intensity curve in the high $Q$ region is determined by the inter-molecular arrangement of a scattering particle, called the form factor. In this region, the internal structure of 
a molecule dominates the scattering. The similarity of the form factor scattering in the SAXS curves implies that the rRNA conformation in the three samples are conserved to the resolution of the SAXS technique. The increased scattering intensity in the low $\mathrm{Q}$ region for the ribosome with structured mRNA is due to the extended conformation of the peripheral protein components. Due to the dominant nature of the rRNA signal in the SAXS measurements, the influence of these extended peripheral ribosomal proteins on the scattering signal is more prominent in the overall size of the particle, the low $\mathrm{Q}$ region, than in the internal structure, the high $\mathrm{Q}$ region. We believe the large particle seen in low $\mathrm{Q}$ of the sample that contains the 70S E. coli ribosome bound to structured mRNA is scattering from the large extended protein components in this sample and that scattering from these components influences this region, and only this region, because scattering from this elongated particle is strong enough only in this low $Q$ region to influence the scattering profile that is dominated by rRNA scattering.

For consistency in our interpretation, SANS data in $42 \% \mathrm{D}_{2} \mathrm{O}$ buffer, a measurement that is sensitive to only RNA scattering, was performed and showed no difference in the RNA structure between the vacant ribosome, the ribosome bound to linear mRNA, and the ribosome bound to structured mRNA (Figure 14 top panel, Table 1). Selectively highlighting protein scattering signal over rRNA, i.e., SANS measurement in $100 \% \mathrm{D}_{2} \mathrm{O}$ buffer, the overall size of the ribosome when bound to structured mRNA is much larger than the ribosome size when vacant or bound to linear mRNA. The increased particle dimensions of the ribosome bound to structured mRNA in $100 \% \mathrm{D}_{2} \mathrm{O}$ confirm an expected extension of protein components. The lack of change in particle dimensions in the $42 \% \mathrm{D}_{2} \mathrm{O}$ measurements that report RNA-only scattering offers further confirmation of this analysis (Figure 14 top panel, Table 1). 


\subsubsection{Characterizing the Conformations of the E. coli 70S Ribosome in Solution.}

To complement scattering data of ribosome in solution with molecular models, known Cryo-EM and X-ray crystallography structures of the ribosome were used to create models. Only the rRNA and ribosomal proteins were used in the building of the vacant ribosome model. For the ribosome samples that are bound to linear or structured mRNA, P-site tRNA and mRNA molecules were also included during the creation of models. The $\mathrm{R}_{g}$ and $\mathrm{D}_{\max }$ of these models were calculated using VMD, Crysol, and Cryson (Table S6 and S7. An extension of the rRNA in solution was observed and compared to rRNA in models. The average $\mathrm{R}_{g}$ of rRNA in the models of the vacant ribosome is $81 \pm 1 \AA$. The scattering data on the vacant ribosome in this study show an $\mathrm{R}_{g}$ of $88 \pm 1 \AA$, an extension of $7 \AA$. This change is inherent to the difference of the assessment technique. SANS is sensitive to the flexible peripheral ribosomal proteins, especially by selectively highlighting protein scattering over the large dominant rRNA scattering. The ribosome size obtained from scattering results is larger than any model (Table S6 and S7) because the scattering from the hydration shell and from the highly flexible, unbound regions of peripheral ribosomal proteins is absent in all the models. The largest change in $\mathrm{R}_{g}$ and $\mathrm{D}_{\max }$ is seen in the SANS data of ribosome in $100 \% \mathrm{D}_{2} \mathrm{O}$ buffer and indicates that the peripheral ribosomal proteins and water interaction play a critical role in the swell of the dimensions. 


\subsection{Discussion}

\subsubsection{Extension of Ribosomal Protein in Ribosomal Helicase Mech- anism}

The 70S ribosome has two different helicase mechanisms. The first mechanism consists of ribosomal interactions that promote an open state of a double-stranded junction in an mRNA secondary structure, likely through interactions by the uS3 protein that stabilize single strandedness of the +12 to +14 nucleotides of an mRNA [19, 32]). This mechanism is known to be passive, fast, and base pair independent [1. 19]. The second mechanism is a mechanical unwinding of the double strand of mRNA that is sitting just outside the mRNA entrance tunnel at +12 . As the ribosome undergoes conformational changes associated with protein translation, the two strands of an mRNA structure are presented as a barrier to translation and must be pulled apart, or unwound. As the GC base pairing in the mRNA structure increases or similarly, if the forces of the mRNA structure are greater, frameshifting is more probable [19, 26]. Thus, this is a tunable mechanism that impacts protein production.

The mRNA:tRNA junction is thought to be the linkage that holds the tension in these active unwinding events and the dependence of frameshifting on tRNA content and dynamics encourages this hypothesis [3, 12]. Many dynamics and structural studies try to understand and extrapolate how the large rRNA structural fluctuations that occur during tRNA translocation are impacted during the active mechanism unwinding $[2,14,27,31,18,19,30,32]$. This study emphasizes the important role of ribosomal proteins in the translational mechanism. Furthermore, this study elucidates the extent of flexibility and extension that the ribosomal proteins can achieve. It provides encouragement to consider ribosomal protein 
flexibility in the assessment of ribosomal dynamics that are observed by smFRET that track fluorophores on ribosomal proteins.

The difference between the extension of the proteins on a ribosome interacting with a structured mRNA compared to a vacant ribosome or a ribosome with linear mRNA shows that large changes can occur in the ribosomal proteins and indicate the importance of these flexible ribosomal proteins in the process of translation. The extension of the ribosomal proteins in this study propose that the ribosomal proteins may play a role in conducting the elusive mechanisms that have been proposed in which the ribosome senses mRNA structures that lie before or after the section of mRNA the ribosome is sitting on [20]. This information of ribosomal protein performance can be applied to the kinetics of -1 programmed ribosomal frameshifting which show an uncoupling of EF-G catalyzed translocation while the ribosome is interacting with a DnaX mRNA hairpin [6], instead of EF-G being heavily mediated by RNA components [14] this study proposes that ribosomal proteins may influence EF-G during these uncoupling events and pauses that are associated with frameshifting.

\subsubsection{Ribosomal Proteins are Flexible and Interact with Water}

Attributes of ribosomal proteins are elusive and are understood by a collection of viewpoints, and this study aims to contribute to this anthology. Ribosomal proteins are known to be intrinsically disordered proteins in solution and undergo a disorder to order transition as they bind to the rRNA. Adaptions seen from thermophilic to mesophilic species show ribosomes maintain high polarity of the rRNA-protein interface, which indicate the disorder of these interfaces and ribosomal proteins are important to be maintained [16]. H/D exchange studies confirm that ribosomal proteins are generally flexible and become more rigid at $\mathrm{Mg}^{+}$con- 
centrations 6 to $10 \mathrm{mM}$ [29]. These studies have a perception that ribosomal proteins can be defined as extensions of the rRNA changes and flexibility. Our study suggests a different view of both of these studies and highlights that the protein should be viewed as independently flexible, and that further study is necessary to compare rRNA vs protein flexibility in order to elucidate dynamical roles.

The SAS scattering reports on the current understanding of the ribosomal proteins as they are found in X-ray crystallography and Cryo-EM structures. Theoretical intensities calculated using these models in Crysol overlays the experimental SAXS curves well without any scaling in Q, but the theoretical intensities calculated using Cryson do not overlay experimental scattering from the ribosome in $100 \% \mathrm{D}_{2} \mathrm{O}$. Though the line shape between the experimental and theoretical curves are very similar, the experimental curve is shifted to lower $\mathrm{Q}$. This indicates that the particle that is observed in the SANS experiment is larger, as if bloated, compared to the theoretical scattering calculated using Cryson using the all-atom models. It is reasonable that the dimensions recorded in this experimental study are due to the extended flexible ribosomal peripheral proteins and the solvent layer [22]. Thus, it is likely that scattering from the hydration shell and from the peripheral ribosomal proteins is not accurately accounted for in modeling or in the calculation of theoretical intensities. The observations of a $3 \AA$ dense water layer seen for a small compact protein [17] may not be accurate for a large, flexible, macromolecular complex such as the ribosome. This must be carefully and thoroughly investigated in order to facilitate the use of SANS as a powerful corroborative technique with the current flourishing output of large, complex, macromolecular structures from Cryo-EM. Specifically, this study indicates that flexible ribosomal proteins are important, yet currently, these proteins and their flexibility are unobserved in static structures of the ribosome. 


\subsection{Methods}

Ribosome Purification Described in Section 2.1.3

Ribosome Characterization Purified ribosomes were compared to PURExpress tight-coupled ribosomes using PURExpress (NEB, E3313). 20 pmoles of each ribosome was used in each reaction, with the concentration of house purified $70 \mathrm{~S}$ ribosomes calculated based on A60 measured by a NanoDrop One (ThermoFischer ND-ONE-W) with 1 A260 unit of 70S ribosome sample is equivalent to 24 $\mathrm{pmol} / \mathrm{mL}$ [7]. In vitro translations were appraised by $4-20 \%$ stain free SDS-PAGE and quantified as the volume of intensity of the produced DHFR protein band at $20 \mathrm{kDa}$.

Small Angle Scattering Experiments tRNA $^{\text {fmet }}$ was bought from MP Biomedicals (Cat 199154, Lot QR15546) and mRNA were RNA oligos purchased from Integrated DNA Technologies (Figure S5). The buffer used in measurements was 20 mM HEPES pH 7.5, $6 \mathrm{mM} \mathrm{MgCL}_{2}, 150 \mathrm{mM} \mathrm{NH}_{4} \mathrm{Cl}, 6 \mathrm{mM} \beta \mathrm{ME}, 2 \mathrm{mM}$ Spermidine, $0.1 \mathrm{mM}$ Spermine. To form ribosome complex with linear or structured mRNA, the ribosomes were mixed at a 1:2:2 molar ratio of ribosome:tRNA:mRNA. Prior to measurements, samples were incubated at $37 \mathrm{C}$ for 5 minutes, then cooled to 10C, equilibrated for at least $5 \mathrm{~min}$ and measured at 10C. SAXS measurements were taken at $\mathrm{LiX}$ beamline (16-ID) at National Synchrotron Light Source II in Brookhaven National Laboratory. SANS measurements were taken at Bio-SANS (CG-3) beamline at the High Flux Isotope Reactor and EQ-SANS (BL-6) at the Spallation Neutron Source in Oak Ridge National Laboratory. Bio-SANS measurements used a neutron wavelength of $6 \AA$ and a sample to detector distance of 15.5 meters. EQ-SANS is on a pulsed-neutron source and the neutron wavelengths and 
detector distances used were $2.5 \AA$ at 1.3 meters, $8 \AA$ at 4 meters, and $10 \AA$ at 4 meters. Sample measurement times were between 30 minutes and 4 hours. Guinier analysis was done in Igor Pro 8 using KCL SAS analysis. Pair-length distributions were found using GNOM [10].

\section{Negative Staining Experiments Described in Section 2.2.3.}

Modeling Only 5S, 16S, and 23S rRNA particles and the ribosomal proteins found in Figure S5 were kept in models of vacant ribosomes. The P-site tRNA and mRNA molecules originally found in PDB structures were kept in the models used to compare with measurements of ribosomes bound to mRNA and P-site tRNA. Crysol and Cryson were used to calculate theoretical scattering intensities, which gave theoretical $\mathrm{R}_{g}$ and $\mathrm{D}_{\max }$ values (Table S6 and S7. Input for Cryson assumed $25 \%$ of hydrogens were nonexchangeable [28].

\section{References}

[1] Hossein Amiri and Harry F Noller. "A tandem active site model for the ribosomal helicase". In: FEBS Letters 593 (2019), pp. 1009-1019. DOI: 10.1002/ 1873-3468 .13383. URL: https : // febs . onlinelibrary . wiley · com/doi / abs/10.1002/1873-3468.13383.

[2] John F Atkins et al. "Ribosomal frameshifting and transcriptional slippage: From genetic steganography and cryptography to adventitious use." eng. In: Nucleic acids research 44.15 (Sept. 2016), pp. 7007-7078. ISSN: 1362-4962 (Electronic). DOI: 10.1093/nar/gkw530. 
[3] Neva Caliskan et al. "Programmed -1 frameshifting by kinetic partitioning during impeded translocation." eng. In: Cell 157.7 (June 2014), pp. 1619-1631. ISSN: 1097-4172 (Electronic). DOI: 10.1016/j .cell.2014.04.041.

[4] Kai-Chun Chang et al. "Resolution-exchanged structural modeling and simulations jointly unravel that subunit rolling underlies the mechanism of programmed ribosomal frameshifting". In: Bioinformatics 35.6 (2018), pp. 945952. ISSN: 1367-4803. DOI: 10 . 1093/bioinformatics/bty762, URL: https : //doi.org/10.1093/bioinformatics/bty762.

[5] Jin Chen et al. "Dynamic pathways of -1 translational frameshifting". In: Nature 512.7514 (Aug. 2014), pp. 328-332. ISSN: 0028-0836. DOI: $10.1038 /$ nature13428. URL: http://europepmc.org/articles/PMC4472451.

[6] Jin Chen et al. "Dynamic pathways of -1 translational frameshifting". In: Nature 512.7514 (Aug. 2014), pp. 328-332. ISSN: 0028-0836. DOI: 10.1038/ nature13428, URL: http://europepmc .org/articles/PMC4472451.

[7] John Christodoulou et al. "Heteronuclear NMR investigations of dynamic regions of intact ${ }_{j} \mathrm{em} \mathrm{m}_{i}$ Escherichia coli ${ }_{i} / \mathrm{em}_{i}$ ribosomes". In: Proceedings of the National Academy of Sciences of the United States of America 101.30 (July 2004), 10949 LP -10954. DOI: 10.1073/pnas . 0400928101. URL: http://www . pnas . org/content/101/30/10949.abstract

[8] Peter V Cornish et al. "Following movement of the L1 stalk between three functional states in single ribosomes". In: Proceedings of the National Academy of Sciences 106.8 (2009), pp. 2571-2576. ISSN: 0027-8424. DOI: 10.1073/pnas. 0813180106. URL: https://www.pnas.org/content/106/8/2571. 
[9] Peter V. Cornish et al. "Spontaneous Intersubunit Rotation in Single Ribosomes". In: Molecular Cell (2008). ISSN: 10972765. DOI: 10.1016/j . molcel. 2008.05.004, arXiv: NIHMS150003,

[10] D Franke et al. " $\{\backslash$ it ATSAS 2.8\}: a comprehensive data analysis suite for small-angle scattering from macromolecular solutions". In: Journal of Applied Crystallography 50.4 (Aug. 2017), pp. 1212-1225. DOI: 10.1107/S1600576717007786. URL: https://doi.org/10.1107/S1600576717007786.

[11] "Ribosomes and Protein Synthesis: A Practical Approach". In: (1990). Ed. by Gary Spedding.

[12] Samuel Hong et al. “Mechanism of tRNA-mediated +1 ribosomal frameshifting". In: Proceedings of the National Academy of Sciences 115.44 (Oct. 2018), 11226 LP -11231. DOI: 10.1073/pnas . 1809319115, URL: http://www . pnas. org/content/115/44/11226. abstract

[13] Tyler Jacks et al. "Signals for ribosomal frameshifting in the rous sarcoma virus gag-pol region". In: Cell 55.3 (1988), pp. 447-458. ISSN: 0092-8674. DOI: http://dx.doi.org/10.1016/0092-8674(88)90031-1. URL: http://www . sciencedirect.com/science/article/pii/0092867488900311.

[14] Hee Kyung Kim and Ignacio Tinoco. "EF-G catalyzed translocation dynamics in the presence of ribosomal frameshifting stimulatory signals". In: $\mathrm{Nu}$ cleic Acids Research 45.5 (2017), pp. 2865-2874. ISSN: 13624962. DOI: 10.1093/ nar/gkw1020.

[15] Mariana Levi and Paul C. Whitford. "Dissecting the Energetics of Subunit Rotation in the Ribosome". In: Journal of Physical Chemistry B 123.13 (2019), pp. 2812-2823. ISSN: 15205207. DOI: 10.1021/acs. jpcb.9b00178. 
[16] Saurav Mallik and Sudip Kundu. "A comparison of structural and evolutionary attributes of Escherichia coli and Thermus thermophilus small ribosomal subunits: signatures of thermal adaptation." eng. In: PloS one 8.8 (2013), e69898. ISSN: 1932-6203 (Electronic). DOI: 10.1371/journal.pone.0069898

[17] Franci Merzel and Jeremy C Smith. "Is the first hydration shell of lysozyme of higher density than bulk water?" eng. In: Proceedings of the National Academy of Sciences of the United States of America 99.8 (Apr. 2002), pp. 5378-5383. ISSN: 0027-8424 (Print). DOI: 10.1073/pnas.082335099.

[18] Peiwu Qin et al. "Structured mRNA induces the ribosome into a hyperrotated state." eng. In: EMBO reports 15.2 (Feb. 2014), pp. 185-190. ISSN: 14693178 (Electronic). DOI: $10.1002 /$ embr.201337762.

[19] Xiaohui Qu et al. "The ribosome uses two active mechanisms to unwind messenger RNA during translation". In: Nature 475 (July 2011), p. 118. URL: https : / / doi . org / 10.1038/nature10126\%20http : / / 10 .0.4.14/ nature $10126 \% 20$ https : / / www . nature . com / articles / nature $10126 \% 7 \mathrm{~B} \%$ 5C\#\%7Dsupplementary-information.

[20] Anna Salvati et al. "Quantitative measurement of nanoparticle uptake by flow cytometry illustrated by an interlaboratory comparison of the uptake of labelled polystyrene nanoparticles". In: NanoImpact 9 (2018), pp. 42-50. ISSN: 2452-0748. DOI: https://doi .org/10 .1016/j . impact . 2017 . 10 .004. URL: http://www.sciencedirect.com/science/article/pii/S2452074817301064.

[21] Ekaterina Samatova et al. "High-efficiency translational bypassing of noncoding nucleotides specified by mRNA structure and nascent peptide". In: Nature Communications 5 (2014), pp. 1-10. ISSN: 20411723. DOI: $10.1038 /$ ncomms5459, URL: http://dx.doi.org/10.1038/ncomms5459. 
[22] Christopher Stanley et al. "Protein structure and hydration probed by SANS and osmotic stress". eng. In: Biophysical journal 94.7 (Apr. 2008), pp. 27772789. ISSN: 1542-0086. DOI: 10 . 1529/biophysj . 107 . 122697, URL: https : //www.ncbi.nlm.nih.gov/pubmed/18178651\%20https ://www.ncbi.nlm. nih.gov/pmc/articles/PMC2267150/

[23] D I Svergun et al. "Solution scattering structural analysis of the $70 \mathrm{~S}$ Escherichia coli ribosome by contrast variation. I. invariants and validation of electron microscopy models11Edited by M. F. Moody". In: Journal of Molecular Biology 271.4 (1997), pp. 588-601. ISSN: 0022-2836. DOI: https ://doi .org/ 10.1006/jmbi.1997.1190. URL: http://www.sciencedirect.com/science/ article/pii/S0022283697911906.

[24] So Umekage and Takuya Ueda. "Spermidine inhibits transient and stable ribosome subunit dissociation". In: FEBS Letters 580.5 (Feb. 2006), pp. 12221226. ISSN: 0014-5793. DOI: $10.1016 / \mathrm{j}$. febslet. 2006.01.033. URL: https : //doi.org/10.1016/j.febslet.2006.01.033

[25] Joseph M Watts et al. "Architecture and secondary structure of an entire HIV1 RNA genome". In: Nature 460.7256 (Aug. 2009), pp. 711-716. ISSN: 00280836. URL: http://dx . doi .org/10.1038/nature08237\%20http://www . nature.com/nature/journal/v460/n7256/suppinfo/nature08237\%7B\%5C_ $\%$ 7DS1.html,

[26] Jin-Der Wen et al. "Following translation by single ribosomes one codon at a time". In: Nature 452.7187 (Apr. 2008), pp. 598-603. ISSN: 0028-0836. DOI: 10.1038/nature06716. URL: http://europepmc.org/articles/PMC2556548.

[27] Ping Xie et al. "Model of the pathway of -1 frameshifting: Long pausing". eng. In: Cell 5.5 (Mar. 2016), pp. 408-424. ISSN: 10974172. DOI: 10.1016/ j . 
bbrep.2016.01.017, URL: http://dx.doi.org/10.1016/j.cell.2014.04. 041\%20http://dx.doi.org/10.1038/ncomms5459\%20http://www.ncbi.nlm. nih.gov/pubmed/14747573\%7B\%5C\%\%7D0Ahttp://www . pubmedcentral.nih. gov/articlerender.fcgi ? artid=PMC369415\%20http : // europepmc . org/ articles/PMC5600365.

[28] Tatsuya Yamamoto, Shunsuke Izumi, and Kunihiko Gekko. “Mass spectrometry of hydrogen/deuterium exchange in $70 \mathrm{~S}$ ribosomal proteins from E. coli." eng. In: FEBS letters 580.15 (June 2006), pp. 3638-3642. ISSN: 0014-5793 (Print). DOI: 10.1016/j.febslet.2006.05.049.

[29] Tatsuya Yamamoto et al. "Mg2+ dependence of $70 \mathrm{~S}$ ribosomal protein flexibility revealed by hydrogen/deuterium exchange and mass spectrometry." eng. In: The Journal of biological chemistry 285.8 (Feb. 2010), pp. 5646-5652. ISSN: 1083-351X (Electronic). DOI: 10.1074/jbc.M109.081836.

[30] Shannon Yan et al. "Ribosome Excursions during mRNA Translocation Mediate Broad Branching of Frameshift Pathways". In: Cell 160.5 (2015), pp. 870881. ISSN: 10974172. DOI: 10.1016/j.cell.2015.02.003.

[31] Shannon Yan et al. "Ribosome excursions during mRNA translocation mediate broad branching of frameshift pathways". In: Cell 160.5 (Feb. 2015), pp. 870-881. ISSN: 0092-8674. DOI: $10.1016 / \mathrm{j}$. cell . 2015 .02 .003. URL: http://europepmc.org/articles/PMC4344849.

[32] Yan Zhang et al. "Alternative Mode of E-Site tRNA Binding in the Presence of a Downstream mRNA Stem Loop at the Entrance Channel". In: Structure 26.3 (2018), 437-445.e3. ISSN: 18784186. DOI: 10.1016/j . str.2018.01.013. URL: https://doi.org/10.1016/j.str.2018.01.013. 


\section{The Influence of mRNA Secondary Structure Stability on Ribo- somal Subunit Rotation}

\subsection{Summary}

The unwinding of structured mRNA by the bacterial 70S ribosome has a hand in dictating frameshifting. Frameshifting is the occurrence of a translating ribosome shifting atop an mRNA strand and the message carried by the mRNA is recoded, often resulting in the production of a different protein or early termination of a protein. The mechanism of frameshifting is harnessed by the cell to diversify the proteome and to deliver precise production ratios. Viruses use frameshifting mRNA structures, so understanding the relationship between the structure of mRNA and frameshifting could aid in disrupting viral infection and in designing CRISPR Cas9 treatments [19, 33]. The ribosome dynamics must be observed during frameshifting to understand impact of mRNA structures on ribosomal dynamics. This study aims to decipher the influence of mRNA structural stability on ribosomal conformation. The ribosome subunit rotation dynamics are observed while the ribosome is unwinding mRNA using single-molecule For̈ster Resonant Energy Transfer.

\subsection{Introduction}

An mRNA molecule acts as a template to be read by the ribosome and plays the role of the skipper as it dictates the order and influences the positioning of tRNA cycling through the ribosome. The anti-codon loop of the tRNA and a codon of the mRNA, each made up of 3 nucleotides, bind to each other and this tRNA:mRNA nucleotide pairing must move through the ribosome without slipping in order for protein elongation to continue in the reading frame. Programmed frameshifting occurs when the tRNA:mRNA nulceotide junction is broken and the ribosome 
continues reading the mRNA within a different reading frame [1, 4]. The fidelity of translation can drop from its usual $99.9 \%$ to around $50 \%$ during programmed frameshifting [4]

The mRNA tunnel through the ribosome is only large enough for linear mRNA to pass through [14, 16, 18, 21, 22, 24, 25, 34, 36]. The mRNA molecules can fold into secondary structures, such as hairpins or pseudoknots. The 70S ribosome is known to have two helicase mechanisms that aid in unwinding structured mRNA. A fast, passive mechanism that promotes mRNA binding to the ribosome as a single strand and an active unwinding mechanism where the rate of unwinding is dependent on the mRNA sequence [3, 32]. The structure and stability of the mRNA secondary structure are correlated with frameshifting efficiency, and the active helicase mechanism is thought to be how the ribosome correlates the mRNA secondary structure to framshifting efficiency.

When the ribosome applies the active helicase mechanism on structured mRNA, it can get stuck in a conformational state and multiple unproductive translocation subunit ratcheting motions are thought to occur [10]. Rates of translocation movements are 10-fold slower than observed in normal translation [8, 10]. The conformational state that the ribosome occupies during these mRNA unwinding events is unclear. The ribosome may occupy a hyper-rotated state where subunit rotation increases about 22 degrees, which is 14 degrees past the rotated (hybrid) state that usually has a 6 degree rotation from the classical non-rotated conformational state [26]. Theoretical studies propose that as the ribosome attempts to unwind a structured mRNA the small subunit rolls about h44 of the $16 \mathrm{~S}$ rRNA by around 12.7 degrees [9].

These studies mentioned above [9, 10, 26] all investigate the translation of the DnaX hairpin. The helicase mechanism is sensitive to changes stability of the hair- 
pin structure [27], which directly impacts the ribosomal frameshifting efficiency [11]. It remains to be determined how ribosome conformational changes as the mRNA structural stability changes. This is addressed using single-molecule Foöter Resonant Energy Transfer (smFRET) to directly investigate the ribosomal conformational changes as mRNA structural stability is varied.

SmFRET is based on the physical phenomena that electronic relaxation can occur through nonradiative energy transfer between two fluorophores. The efficiency of this energy transfer is related to the distance between the two fluorophores. This distance-dependent energy transfer phenomenon is called Forster Resonant Energy Transfer (FRET) and is a powerful tool in understanding changes between two points in inter-molecular or intra-molecular space. Fluorophores can be attached to molecules, and FRET efficiency can be used to measure distances on the nanometer length scale by using a Total Internal Reflection (TIRF) Microscopy [6. 17. 28]. The ribosome is an optimal nano-machine to investigate with smFRET due to its size, flexibility, and thorough structural and functional characterization [30. 26].

\subsection{Methods}

\subsubsection{Creation of Macromolecular Components}

mRNA synthesis RNA oligos were synthesized using T7 RNA polymerase in vitro translation and purified using two steps of gel electrophoresis, specifically using Urea PAGE electrophoresis and Whatman®elutrap [2, 31]. The RNA was run on a Urea PAGE gel and identified with Ultraviolet shadowing. The RNA band was cut out and put in an elutrap reservoir filled with $1 \mathrm{M}$ Tris $\mathrm{pH} 8,1 \mathrm{M}$ Boric acid and $0.02 \mathrm{M}$ EDTA (1X TBE buffer) and set up according to Whatman®instructions. The Elutrap was run overnight at 300 Volts and the eluted RNA was concentrated 
and flash frozen in liquid nitrogen in RNAse free eppindorph tubes and kept and $-80^{\circ} \mathrm{C}$. RNAse enzymes promote the breakdown of RNA and their presence is ubiquitous and their stability robust. Exhaustive measures must be taken to minimize the exposure or RNAses to the RNA Oligos. All tubes and tips must be RNAse free, and equipment must be thoroughly washed and dried in a clean oven.

Labeled Ribosomes Incorporation of dyes into ribosomes was done using previously labeled uS6 and uL9 [12, 30]. Reconstituting labeled proteins was done by the addition of uS6 and uL9 to ribosomes that have Cy3-uS6 and Cy5-uL9 deletions. The incorporation of dyes into ribosomes can be quantified by measuring the absorbance of Cy3, at $550 \mathrm{~nm}$ with an extinction coefficient of 136,000, Cy5, as $649 \mathrm{~nm}$ with an extinction coefficient of 250,00013.

$$
\frac{(\text { Absorbance })(\text { pathlength })(\text { Dilution })}{\text { Extinction Coefficient }}
$$

Pathlength is the distance that light passes through the sample and is often recorded in centimeters. Absorbance units are in AU. Ribosomes concentration can be found using absorbance at $260 \mathrm{~nm}$ where $1 \mathrm{AU}$ is the equivalent of 400 moles/ul. The location of $\mathrm{Cy} 3$ and $\mathrm{Cy} 5$ can be found by running a tagged ribosome on an SDSPAGE gel and imaging at the excitation wavelength. If the fluorescence is concentrated in a single band, it is considered correctly incorporated (Figure 54).

Macromolecular Complex Formation To make ribosome complexes the mRNA oligo and tRNA was bound and incubated with the labeled ribosomes for 30 minutes at $37^{\circ} \mathrm{C}$. tRNA ${ }^{\text {fmet }}$ was bought from MP Biomedicals. The concentrations of each component were $0.25 \mathrm{uM} 70 \mathrm{~S}, 0.5 \mathrm{uM}$ mRNA oligo, and $1 \mathrm{uM}$ tRNA $^{\text {fmet }}$. After incubation, the complexes were applied to the slides and imaged 
within an hour of heating step.

\subsection{2 smFRET Microscopy and Analysis}

Slide Preparation Quartz slides are often used to house the experimental sample in microscopies, such as Total Internal Reflection Fluorescence (TIRF) that use light or other radiation. Quartz is an ideal candidate to hold the sample due to its crystalline nature, and the refractive index (about 1.5) can be easily matched with an oil. This allows a microscope set up that can observe a sample without having a dielectric interface between outside the slide and the slide itself. Unfortunately, Quartz will non-specifically bind macromolecules, which, in most experimental setups, can ruin both the sample contents and the signal from the sample. Passivation of slides is done to decrease non-specific binding. Common practices use a layer of polyethelene glycol (PEG) [12, 20, 30], Tween-20 [2], or Bovine Serum Albumin (BSA) [7] coated onto the slide to prevent non-specific binding. Slides must be extensively cleaned, and kept clean, for good signal to noise in fluorescence data as any impurities will fluoresce. Slides used for this work have three flow chambers constructed on each slide between a glass coverslip and a quartz slide that was passivated using PEG [12, 30].

Tethering Assemblies to the Slide The field of the evanescent wavefront that penetrates the coverslip provides the energy for fluorophores to fluoresce, this wave reaches about $100 \mathrm{~nm}$ beyond the coverslip with this experimental setup [17. [30]. The complex of interest must be attached to keep the molecule within about $100 \mathrm{~nm}$ of the coverslip (Figure 15). Once the slide is passivated, tethering is done by linking polymers. In this study, the mRNA is used to tether the complex to the slide. mRNA is annealed to a DNA oligo by heating for 5 minutes at $65^{\circ} \mathrm{C}$ before it 
is bound to a ribosome. The DNA oligo has a biotin molecule covalently attached to its $5^{\prime}$ end (5' biotin - CTTTATCTTCAGAAGAAAAACC-3' bought from IDT). Biotin is a small molecule that binds tightly to a small protein named avidin. In these experiments, a fraction of the PEG used during passivation had avidin attached to it. Biotin-tagged ribosome complexes are incubated on the slide, and the biotin and avidin bind together to tie the complex to the slide (Figure 15). The fraction of PEG-avidin and PEG used in passivation can be tuned such that about 100 single molecules are in the view field of the microscope per measurement.

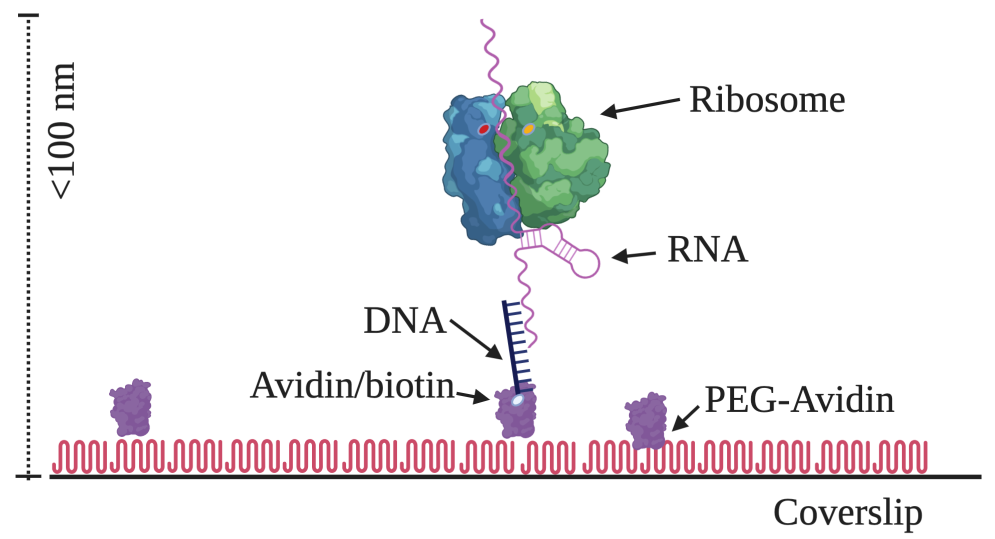

Figure 15: Schematic of tethering a ribosome complex in an smFRET experiment.

Total Internal Reflection Microscopy measurements Measurements were taken on a homemade TIRF microscope equipped with an Acousto Optic Tunable Filter (AOTF) and a 60X oil objective lens. The AOTF is used to focus and set the intensity of the fluorescence signal. Care must be taken to not get bubbles in the oil between the lens and the slide and to close the shutter, as this will ruin signal. A prism directs lasers to hit the interface between coverslip and sample. The incoming laser must hit at a precise angle so that the entire beam is internally reflected. When the light is totally reflected by a solid/liquid interface, an electromagnetic field called an evanescent wave is made [17]. In this way, energy can reach and 
excite the fluorophores because the energy carried by the wave is equivalent to the energy of the laser wavelength of the reflected beam [5, 6]. Fluorescence from the donor and acceptor fluorophore are split into two pathways for separate detection with a dichroic mirror. Photos are captured as time-stamped movies by a camera. This study uses an Andor iXonEM+897 Electron Multiplying Charge Coupled Device (EMCCD) camera, with care taken not to over saturate the camera at any given time. This method uses crimson fluorescent FluoSpheres®(Invitrogen) for TIRF microscope calibration and a tool in aiding software processing after movies capturing fluorescence are taken on the microscope.

Analysis of smFRET movies Fluorescent readings from single molecules are extracted from the movies taken on a TIRF microscope. Analysis is done with homemade software. Software identifies areas of fluorescence within each movie and extracts the detected fluorescence to provide a temporal fluorescent trace from single particles. The fluorescence from the donor and acceptor can be used to calculate the approximate FRET efficiency ( $E_{a p p}$, Equation 14 [ [28]

$$
\frac{I_{A}}{I_{A}+I_{D}}=E_{a p p}
$$

The calculated $E_{a p p}$ from this equation does not account for uncertainty in the anisotropy of the fluorophores. Instrumental corrections must be applied in order to properly calculate FRET, which is a correction factor denoted $\gamma$ (Equation 16.

$$
\gamma=\Delta I_{A} I \Delta I_{D}
$$

Where $\gamma$ is the ratio of change in donor and acceptor intensity upon photobleaching, respectively $\Delta I_{D}$ and $\Delta I_{A}$. This is applied to find a corrected FRET efficiency 
$E$ in Equation [16][28].

$$
E=\left[1+\gamma \frac{I_{D}}{I_{A}}\right]^{-1}
$$

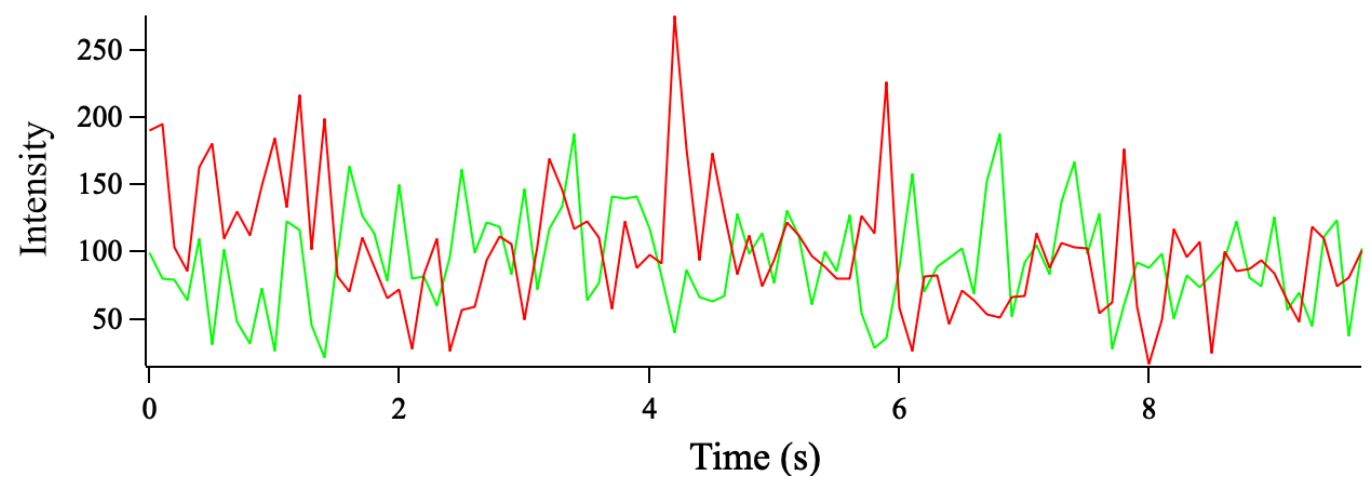

Figure 16: Example of a trace where $I_{A}$ and $I_{D}$ are plotted over time.

The data can be appraised as the overlay of $I_{D}$ and $I_{A}$ or calculated E (Figure 16). The plot of $I_{D}$ and $I_{A}$ that is emitted from a single particle over time is referred to as a trace (Figure 16). Traces must be sorted and validated as every trace may not show FRET or proper fluorescent interaction. Different criteria are often used to sort traces that will be used to calculate FRET. These criteria are often implemented by sorting algorithms, manual appraisal, or both. There is not a widely implemented algorithm to sort traces from smFRET measurements [28]. This study uses manual appraisal. Criteria for manual appraisal for particle picking are often a minimum of data points, a minimum of a FRET state observed in a trace, clear photobleaching, and the most reliable indication of FRET is a positive correlation between $I_{A}$ and $I_{D}$ (Figure 16). FRET is calculated from these traces and plotted as a histogram that shows the entire FRET amount of each FRET seen (Figure 18). 


\subsection{Results and Discussion}

\subsubsection{Visualization of Subunit Population During Helicase Activity}

This study investigates mRNA influence on ribosome subunit rotation. DnaX mRNA provided the control for an mRNA with secondary structure and $m 291$ mRNA provided the control for an mRNA with no secondary structure (Table S5) [29]. DnaX mRNA has been shown to induce a large population to a 0.2 FRET state using this instrumental and experimental setup [26]. This FRET state is noted as the hyper-rotated state because it is thought to be the observation of extended subunit rotation by about $16 \%$ past the rotation state observed in crystal structures. The hyper-rotated state is not observed while the ribosome is bound to linear mRNA. The DnaX mRNA hairpin was redesigned to destabilize the top half or bottom half of the hairpin, respectively named BS2 and BS3 (Figure 17). All mRNA

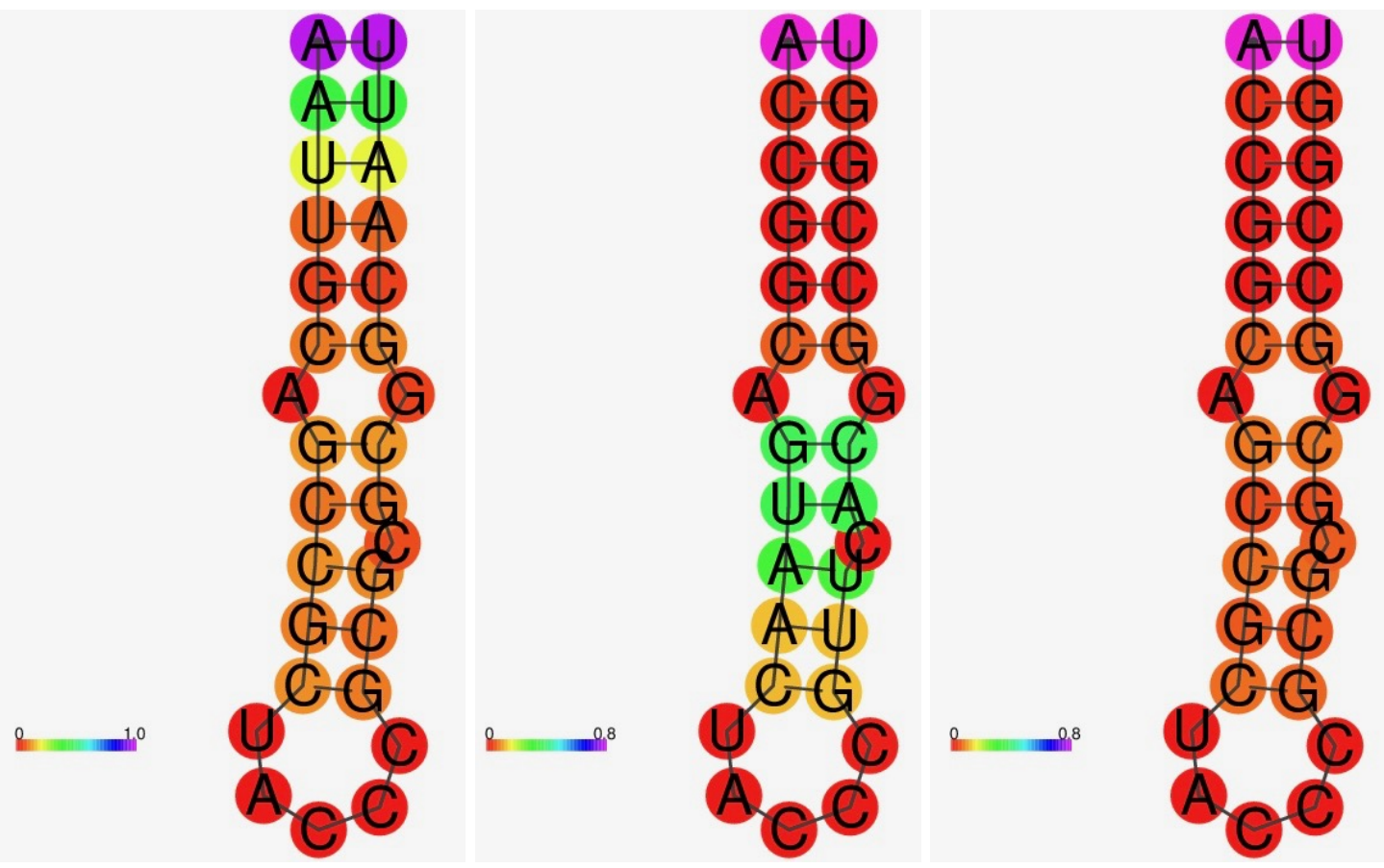

Figure 17: The three hairpins are shown as MFE structure [23]. Left: BS2, Middle: BS3, Right: DnaX. 
hairpins sit just outside the ribosome mRNA entrance tunnel and will interact with the active helicase mechanism, which is about +11 to +12 base pairs away from the P-site [11, 26, 30, 32]. The positional entropy is indicated by hue with red as a welldefined position to green being an ill-defined position that could be an incorrect structure or a disordered segment. The region of the hairpin closer to the ribosome may even be single-stranded in BS2 (left, Figure 17) and the top of the hairpin may not be bound as a hairpin in BS3 (middle, Figure 17) whereas it is likely the entire hairpin forms for DnaX (right, Figure 17). By observing ribosome subunit rotation complexed with destabilized hairpin structures, it is expected to see a lower population of the 0.2 hyper-rotated FRET state. Traces that portray the sorting criteria
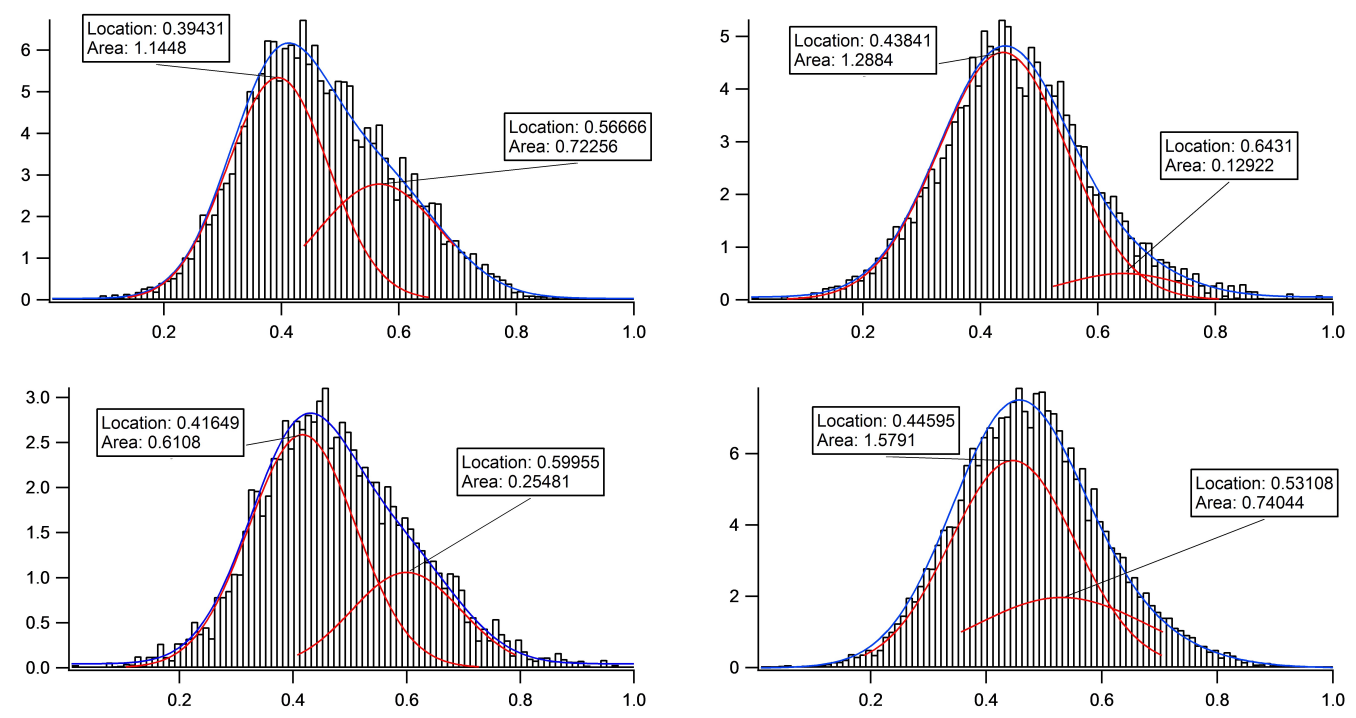

Figure 18: smFRET histograms of m291 (top left), BS2 (top right), BS3 (bottom left), and DnaX (bottom right).

are clipped before a photobleaching event, and the calculated FRET from selected traces are then binned to create histograms. The experiment tracks the ratchet-like rotation of the $50 \mathrm{~S}$ and $30 \mathrm{~S}$ subunits and reports the 0.6 FRET is a non-rotated subunits and 0.4 FRET is rotated subunits [12, 26, 30]. FRET histograms from past studies using this experimental setup with separate population peaks at 0.4 and 
0.6 and a peak or shoulder at 0.2 FRET state. The populations of these FRET states are not distinct in this study (Figure 18). Although the histograms can be fit using two populations, the FRET populations differ in their location between the four mRNA tested.

\subsection{Future Work}

Though the atomic coordinates can influence the smFRET output and interpretation of dynamics [35] it is unlikely the experimental design is the origin of the ambiguous experimental output due to the robust use of the experimental design [12, 26]. Extensive measures were taken to decrease the signal to noise ratio in an attempt to deconvolute the populations; including optimizing slide preparation procedures, laser intensity, and sample preparation. Future work for this project would be to further decrease experimental background noise and to hone the manual procedure of sorting traces. Sorting contains multiple manual procedures that can introduce uncertainty is the reported FRET populations. General appraisal must be done by eye to identify FRET between $I_{D}$ and $I_{A}$. Thousands of traces are sorted through by eye over the span of multiple days. The acceptance or rejection of a trace is dependent on a caliber set by a human and their eye. Traces that look like FRET have other manipulations done by eye such as setting the background intensity of fluorescence and clipping the trace after photobleaching.

Some uncertainties associated with the handling of smFRET traces have been addressed by the field. Experimental methods have been developed to address background signal uncertainty; for example, Zero-Mode Waveguides are nanostructures that isolate signal from single molecules [10, 13, 15]. Though, it remains necessary for the field to invest in accuracy and reproducibility in sorting traces. Each time a novel dynamic mode of the ribosome is investigated, the sample and 
experimental design is recreated. Specifically, to appraise a new dynamic motion of the ribosome the translation machinery must have fluorophores placed in new locations. Changing the location of donor and acceptor fluorophores will influence $I_{D}$ and $I_{A}$, and the FRET populations reported. Creating universal standards and methods of trace sorting is challenging to implement due to the variability in $I_{D}$ and $I_{A}$ as experiment designs evolve.

\section{References}

[1] Vivek M Advani and Jonathan D Dinman. "Reprogramming the genetic code: The emerging role of ribosomal frameshifting in regulating cellular gene expression." eng. In: BioEssays : news and reviews in molecular, cellular and developmental biology 38.1 (Jan. 2016), pp. 21-26. ISSN: 1521-1878 (Electronic). DOI: 10.1002/bies.201500131.

[2] Yasar Luqman Ahmed and Ralf Ficner. "RNA synthesis and purification for structural studies." eng. In: RNA biology 11.5 (2014), pp. 427-432. ISSN: 15558584 (Electronic). DOI: 10.4161/rna.28076.

[3] Hossein Amiri and Harry F Noller. "A tandem active site model for the ribosomal helicase". In: FEBS Letters 593 (2019), pp. 1009-1019. DOI: 10.1002/ 1873-3468.13383, URL: https : // febs . onlinelibrary · wiley . com/doi/ abs/10.1002/1873-3468.13383.

[4] John F Atkins et al. "Ribosomal frameshifting and transcriptional slippage: From genetic steganography and cryptography to adventitious use." eng. In: Nucleic acids research 44.15 (Sept. 2016), pp. 7007-7078. ISSN: 1362-4962 (Electronic). DOI: 10.1093/nar/gkw530. 
[5] D Axelrod. "Cell-substrate contacts illuminated by total internal reflection fluorescence." eng. In: The Journal of cell biology 89.1 (Apr. 1981), pp. 141-145. ISSN: 0021-9525 (Print). DOI: 10.1083/jcb.89.1.141.

[6] D Axelrod. "Total internal reflection fluorescence microscopy in cell biology." eng. In: Traffic (Copenhagen, Denmark) 2.11 (Nov. 2001), pp. 764-774. ISSN: 1398-9219 (Print).

[7] D W Bo Broadwater Jr et al. “ERASE: a novel surface reconditioning strategy for single-molecule experiments". In: Nucleic Acids Research 47.3 (Nov. 2018), e14-e14. ISSN: 0305-1048. DOI: 10.1093/nar/gky1168. URL: https ://doi . org/10.1093/nar/gky1168.

[8] Neva Caliskan et al. "Programmed -1 frameshifting by kinetic partitioning during impeded translocation." eng. In: Cell 157.7 (June 2014), pp. 1619-1631. ISSN: 1097-4172 (Electronic). DOI: 10.1016/j .cell.2014.04.041.

[9] Kai-Chun Chang et al. "Resolution-exchanged structural modeling and simulations jointly unravel that subunit rolling underlies the mechanism of programmed ribosomal frameshifting." eng. In: Bioinformatics (Oxford, England) 35.6 (Mar. 2019), pp. 945-952. ISSN: 1367-4811 (Electronic). DOI: 10 . $1093 /$ bioinformatics/bty762.

[10] Jin Chen et al. "Dynamic pathways of -1 translational frameshifting". In: Nature 512.7514 (Aug. 2014), pp. 328-332. ISSN: 0028-0836. DOI: 10.1038/ nature13428, URL: http://europepmc.org/articles/PMC4472451.

[11] Jin Chen et al. "Dynamic pathways of -1 translational frameshifting". In: Nature 512.7514 (Aug. 2014), pp. 328-332. ISSN: 0028-0836. DOI: $10.1038 /$ nature13428, URL: http://europepmc.org/articles/PMC4472451. 
[12] Peter V. Cornish et al. "Spontaneous Intersubunit Rotation in Single Ribosomes". In: Molecular Cell (2008). ISSN: 10972765. DOI: 10.1016/j . molcel. 2008.05.004, arXiv: NIHMS150003.

[13] Garrison M Crouch, Donghoon Han, and Paul W Bohn. "Zero-mode waveguide nanophotonic structures for single molecule characterization". In: Journal of Physics D: Applied Physics 51.19 (2018), p. 193001. ISSN: 0022-3727. DOI: 10 . 1088/1361-6463/aab8be. URL: http://dx . doi .org/10 . 1088/13616463/aab8be.

[14] Gabriel Demo et al. "Mechanism of ribosome rescue by ArfA and RF2". In: eLife 6 (2017). Ed. by Rachel Green, e23687. ISSN: 2050-084X. DOI: 10.7554/ eLife.23687, URL: https://doi.org/10.7554/eLife.23687,

[15] Olivier Duss et al. "Real-time assembly of ribonucleoprotein complexes on nascent RNA transcripts". In: Nature Communications 9.1 (2018), p. 5087. ISSN: 2041-1723. DOI: 10.1038/s41467-018-07423-3. URL: https://doi .org/10. 1038/s41467-018-07423-3.

[16] Shu Feng, Yun Chen, and Yong Gui Gao. "Crystal Structure of 70S Ribosome with Both Cognate tRNAs in the E and P Sites Representing an Authentic Elongation Complex". In: PLoS ONE 8.3 (2013), pp. 1-11. ISSN: 19326203. DOI: 10.1371/journal.pone.0058829

[17] Kenneth N Fish. "Total internal reflection fluorescence (TIRF) microscopy". eng. In: Current protocols in cytometry Chapter 12 (Oct. 2009), Unit12.18-Unit12.18. ISSN: 1934-9300. DOI: 10.1002/0471142956 . cy1218s50. URL: https ://www . ncbi.nlm.nih.gov/pubmed/19816922\%20https://www.ncbi.nlm.nih.gov/ pmc/articles/PMC4540339/. 
[18] Haixiao Gao et al. "Study of the Structural Dynamics of the E. coli 70S Ribosome Using Real-Space Refinement Georgia Institute of Technology". In: Cell 113 (2003), pp. 789-801.

[19] David P Giedroc and Peter V Cornish. "Frameshifting RNA pseudoknots: Structure and mechanism". In: Virus Research 139.2 (2009), pp. 193-208. ISSN: 0168-1702. DOI: https://doi.org/10.1016/j virusres .2008.06.008, URL: http://www.sciencedirect.com/science/article/pii/S0168170208002323.

[20] Chirlmin Joo and Taekjip Ha. "Preparing sample chambers for single-molecule FRET." eng. In: Cold Spring Harbor protocols 2012.10 (Oct. 2012), pp. 11041108. ISSN: 1559-6095 (Electronic). DOI: 10.1101/pdb.prot071530.

[21] Patricia Julián et al. "Structure of ratcheted ribosomes with tRNAs in hybrid states". In: Proceedings of the National Academy of Sciences of the United States of America 105.44 (2008), pp. 16924-16927. ISSN: 00278424. DOI: 10.1073/pnas. 0809587105 ,

[22] Serdal Kirmizialtin et al. Using molecular simulation to model high-resolution cryo-EM reconstructions. 1st ed. Vol. 558. 1. Elsevier Inc., 2015, pp. 497-514. DOI: 10.1016/bs.mie.2015.02.011. URL: http://dx.doi.org/10.1016/bs. mie.2015.02.011.

[23] Ronny Lorenz et al. "ViennaRNA Package 2.0." eng. In: Algorithms for molecular biology : AMB 6 (Nov. 2011), p. 26. ISSN: 1748-7188 (Electronic). DOI: 10. $1186 / 1748-7188-6-26$.

[24] Anna B Loveland and Andrei A Korostelev. "Structural dynamics of protein S1 on the 70S ribosome visualized by ensemble cryo-EM". In: Methods (San Diego, Calif.) 137 (Mar. 2018), pp. 55-66. ISSN: 1095-9130. DOI: 10.1016/ j. 
ymeth . 2017 . 12.004. URL: https : / / www . ncbi . nlm . nih . gov/pubmed/ 29247757\%20https://www.ncbi.nlm.nih.gov/pmc/PMC5866760/.

[25] Soneya Majumdar et al. "Disrupting domain-domain interactions is indispensable for EngA-ribosome interactions". In: Biochimica et Biophysica Acta Proteins and Proteomics 1865.3 (2017), pp. 289-303. ISSN: 18781454. DOI: 10 . 1016 / j . bbapap . 2016 .12.005. URL: http : / / dx . doi .org/10 . 1016/j . bbapap.2016.12.005.

[26] Peiwu Qin et al. "Structured mRNA induces the ribosome into a hyperrotated state." eng. In: EMBO reports 15.2 (Feb. 2014), pp. 185-190. ISSN: 14693178 (Electronic). DOI: $10.1002 /$ embr . 201337762.

[27] Xiaohui $\mathrm{Qu}$ et al. “The ribosome uses two active mechanisms to unwind messenger RNA during translation". In: Nature 475 (July 2011), p. 118. URL: https : / / doi .org / 10 . 1038 / nature10126\%20http : / / 10 . 0 . 4.14 / nature10126\%20https : / / www . nature . com / articles / nature10126\%7B \% 5C\#\%7Dsupplementary-information.

[28] Rahul Roy, Sungchul Hohng, and Taekjip Ha. "A practical guide to singlemolecule FRET". eng. In: Nature methods 5.6 (June 2008), pp. 507-516. ISSN: 1548-7105. DOI: $10.1038 /$ nmeth . 1208, URL: https : / / wwW . ncbi .nlm . nih . gov/pubmed/18511918\%20https://www.ncbi.nlm.nih.gov/pmc/articles/ PMC3769523/.

[29] Bassem Shebl. “The Influence of mRNA Secondary Structure on Ribosome Conformational Dynamics". PhD thesis. University of Missouri, 2016.

[30] Bassem Shebl et al. "Preparation of ribosomes for smFRET studies: A simplified approach". In: Archives of Biochemistry and Biophysics 603 (2016), pp. 118130. ISSN: 0003-9861. DOI: http : / / dx . doi . org/10 . 1016/j · abb . 2016 . 
05. 010. URL: http : / / wwW . sciencedirect . com/science/article / pii / S0003986116301576.

[31] Heike Summer, Rene Gramer, and Peter Droge. “Denaturing urea polyacrylamide gel electrophoresis (Urea PAGE)." eng. In: Journal of visualized experiments : JoVE 32 (Oct. 2009). ISSN: 1940-087X (Electronic). DOI: 10.3791/1485.

[32] Seyedtaghi Takyar, Robyn P Hickerson, and Harry F Noller. "mRNA helicase activity of the ribosome." eng. In: Cell 120.1 (Jan. 2005), pp. 49-58. ISSN: 0092-8674 (Print). DOI: 10.1016/j.cell.2004.11.042.

[33] Joseph M Watts et al. "Architecture and secondary structure of an entire HIV1 RNA genome". In: Nature 460.7256 (Aug. 2009), pp. 711-716. ISSN: 00280836. URL: http : / / dx . doi .org/10 . 1038/nature08237\%20http : / / www . nature.com/nature/journal/v460/n7256/suppinfo/nature08237\%7B\%5C_ $\% 7 \mathrm{DS} 1 . \mathrm{html}$.

[34] Paul C. Whitford and Karissa Y. Sanbonmatsu. "Simulating movement of tRNA through the ribosome during hybrid-state formation". In: Journal of Chemical Physics 139.12 (2013), pp. 1-9. ISSN: 00219606. DOI: 10 . 1063 / 1. 4817212 .

[35] Paul Charles Whitford. “The ribosome's energy landscape: Recent insights from computation". eng. In: Biophysical reviews 7.3 (Jan. 2015), pp. 301-310. ISSN: 1867-2450. DOI: 10 . 1007 / s12551-014-0155-1. URL: https : / / wWW . ncbi.nlm.nih.gov/pubmed/28510226\%20https://www.ncbi.nlm.nih.gov/ pmc/PMC5418421/.

[36] Yan Zhang et al. "Alternative Mode of E-Site tRNA Binding in the Presence of a Downstream mRNA Stem Loop at the Entrance Channel". In: Structure 
26.3 (2018), 437-445.e3. ISSN: 18784186. DOI: $10.1016 /$ j . str . 2018 . 01 . 013.

URL:https://doi.org/10.1016/j.str.2018.01.013. 


\section{Probing the Bacterial Co-Translational Translocation Pathway}

\subsection{Summary}

Co-translational translocation occurs by having a ribosome bind to a translocon and synthesize a protein into or through the membrane; this is thought to be the main pathway that produces integral membrane proteins (IMPs). Components and involvement in co-translational translocation have been investigated, yet some kinetics and dynamics of the pathway remain unclear [12, 18, 24]. The objective of this study is to understand the influence of the ribosome delivery path on the function, structure, and stoichiometry of the co-translation translocation complex. In this study, we recreate the pathway in vitro in order to observe the impact of pathway components on IMP production and to image the co-translational translocation system using tapping mode Atomic Force Micrsocopy (AFM). The work successfully recreates components of the pathway in vitro and images components using AFM. Future work in this study includes future optimization of output assessment of the in vitro co-translational translocation assay and AFM imaging conditions. The elucidated information found is applicable to IMP creation in many organisms as the pathway is ubiquitously found in nature.

\subsection{Introduction}

Integral membrane proteins (IMPs) are cellular gatekeepers that transport information and molecules across the plasma membrane of all cells. Without biosynthesis that properly creates IMPs the cell would not interact with its external environment or productively control its internal environment. It is generally recognized that the majority of IMPs on the plasma membrane are manufactured via a universally conserved pathway called co-translational translocation, though the 
exact amount of proteins that use the pathway remains undetermined [17]. The main components of this pathway are macromolecular complexes made up of the protein-synthesizing ribosome and the protein-conducting translocon. The integration of these two components is achieved by a Signal Recognition Particle (SRP) and the SRP in E. coli cells is called FtsY [1]. The macromolecules involved in the SRP-mediated co-translational translocation pathway rely on the binding kinetics of their individual parts, which allows for many sub-populations and intermediates that are difficult to detect in bulk biochemistry experiments [12, 26, 27]. Thus, single-molecule experiments are necessary to resolve the structure, function, and dynamics of these complex systems and correlate them to the product they create. This project aims to observe and quantify the impact FtsY has on IMP creation via an in vitro assay and to observe the SecYEG:Ribosome complex as made by FtsY using the single-molecule approach of tapping mode AFM imaging.

Much is known about the insertion of IMPs with the ribosome:translocon complex. The parameters that affect membrane insertion of a transmembrane spanning region of a protein are hydrophobicity of its core, flanking charges, and the rate at which the nascent chain is translated. It has been shown that the orientation of the signal peptide is determined within the first 50 seconds of the initiation of cotranslational translocation [8]. Molecular Dynamics simulations also suggest that the rate at which the nascent peptide is elongated impacts the orientation of transmembrane segments [27]. These studies propose that the dynamics and kinetics of the ribosome and translocon manage peptide insertion under the guidance of the nascent peptide. Previous studies show that ribosomes do not need SRP components to bind to SecYEG [26]. This study aims to study the ribosome-translocon machinery as a function of the creation of the complex with and without the use of FtsY. This is done by recreating the pathway in vitro and imaging the system 
with Atomic Force Microscopy to understand the direct influence of FtsY on complex formation. Scanning mode AFM in tapping mode is used to investigate cotranslational translocation machinery as this method allows for visualization of the topology of macromolecules submerged in liquid with little distortion.

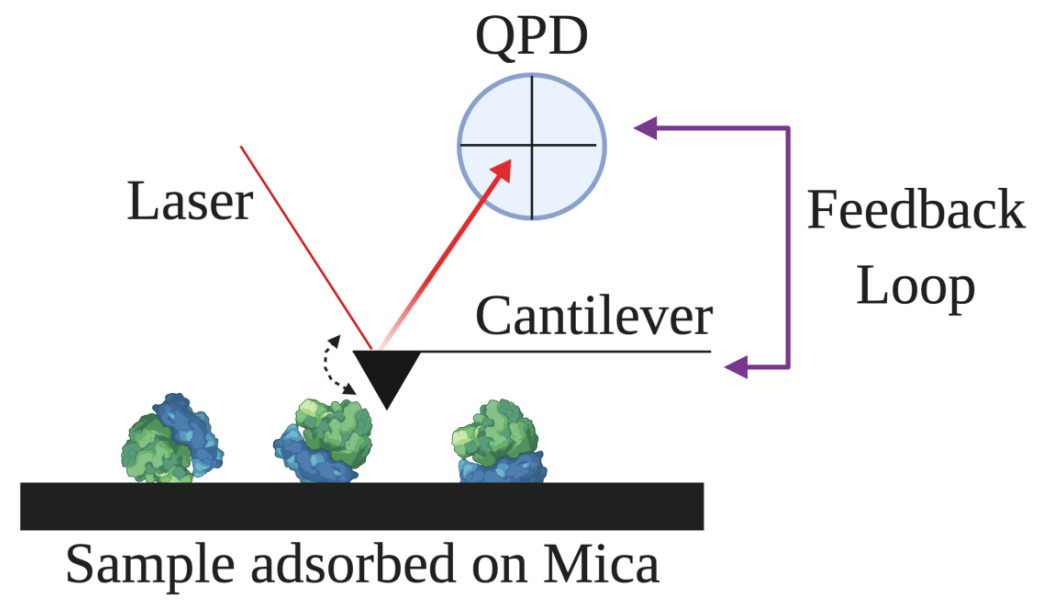

Figure 19: Setup of an Atomic Force Microscope with tapping motion visualized.

Components of the AFM are a cantilever with an atomically sharp tip on the end that physically probes a sample, a laser source that deflects off the cantilever, a quadrant photo-diode (QPD) for optical detection of the deflected laser, as well as piezoelectrics and electronics that provide appropriate movement of components in response to user input and instrument feedback (Figure 19). In scanning mode AFM the oscillating tip is moved, or scanned, over a surface. This microscopy yields an image of a specimen but is distinctly different from traditional imaging that uses radiation as a probe. Comparing the electron microscopy (EM, Section 2.2.3) with scanning mode AFM, an EM image yields a two-dimensional projection whereas scanning mode AFM yields a topological map, containing information about the surface such as roughness and height changes [3]. During imaging, the main forces that perturb a tip are Van der Waals and electrostatic interactions [16]. When applying this method to hydrated, soft matter, considerations on envi- 
ronmental salts and tip design need to be carefully considered. In order to gently probe a macromolecule, a highly flexible cantilever must be used so that even a small force on the tip can produce a change in cantilever orientation and be detected by the QPD without destroying the molecule [2]. In order for a macromolecule to stay folded while adsorbed to a surface and for the forces that keep the molecule correctly folded and functional, proper salt and imaging conditions must be found.

\subsection{Methods}

The development of this study began with two routes. The first is the creation of in vitro co-translational translocation in an assay reaction. The second is developing the technique to image the Ribosome:SecYEG complex using AFM.

\subsubsection{In vitro Co-translational Translation Assay Development}

The ribosome gets trafficked to the translocon after a nascent peptide has already begun to be translated (called a ribosome nascent chain complex or RNC) [1, 12, 18, 26, 27]. Thus, the ribosome must first be started and stalled in vitro for the ribosome:translocon to assemble during the recreation of this pathway. The components for this in vitro co-translational translation assay are a stalled ribosome nascent chain complex, the chaperone FtsY, either an inner membrane vesicles or a proteoliposome containing SecYEG, and a translation mixture. The translation mixture provides components of translation such as aminoacylated tRNA and elongation factors. Two types of RNCs were made, a rescuable RNC and a non-rescuable RNC. The rescuable RNC can be used to create IMPs in the in vitro co-translational translation assay and the non-rescuable RNC is cheaper and easier

to make and will be used to test binding and AFM imaging optimization. By re- 
creating co-translational translocation in vitro the activity of the ribosome:SecYEG complex as a function of FtsY delivery can be tested. The output from this assay will be a measurement of the nascent chain length and quantity. Components of this assay will create samples that will be imaged with AFM.

Ribosome Two ribosome preparations were performed. The first is a ribosome made for RNC production by a single step of affinity chromatography [4]. This method was done to produce a large amount of highly functional ribosomes to use in the reactions described below in RNC production. The second was a method developed for extremely pure ribosomes to be imaged with AFM (Section 2.1.3).

RNC production A stalled and rescuable ribosome was created in order to decouple the kinetics of protein initiation or the impact that the nascent chain length has on RNC binding to SecYEG. The decoupling of these kinetics will allow for the observation of the kinetics of FtsY dependent delivery [18]. Ribosomes were made into RNC by a reaction mixture that contained ribosomes, a translation mixture, and the DNA to be transcribed/translated.Two stalling sequences were constructed. The non-rescuable RNC used SecM sequence that will stall the ribosome during translation [20, 28] through interactions between the nascent peptide and the ribosome. The DNA that was used to make the non-rescuable RNC uses the C terminal leader sequence ${ }^{150}$ FSTPVWISQAQGIRAGP ${ }^{166}$ of SecM. This sequence stalls the ribosome and targets the ribosome to be picked up by chaperone FtsY and brought to the SecYEG. The sequence was cloned with the primers (Table 2). The translation mixture for non-rescuable RNC can be S30 mix which contains all components of translation. The non-rescuable RNC is stalled using a stop codon (AUG) with a translation mixture that does not contain the release factor that binds the AUG codon, RF1. The additional component of the rescuable RNC is a sup- 
Table 2: Primers used to clone SecM.

\begin{tabular}{l|r} 
Primer Type & Sequence \\
\hline Forward & 5' CATCCGTGCTGGCCCTTAGCGCCTCACCTAACAAC 3' \\
Reverse & 5' GTTGTTAGGTGAGGCGCTAAGGGCCAGCACGGATG 3'
\end{tabular}

pressor tRNA that binds to AUG and provides an amino acid, in this case, a serine, in order to restart translation [22]. PURExpress system that contains all necessary components for translation except RF1. The SecM sequence was modified to take out the proline that is necessary for stalling and to insert an alanine in this place [28]. An AUG codon [29] was added that sits in the ribosomal A-site, such that both the rescuable and non-rescuable RNC stall with the same nascent chain length. The primers used for point mutations in these sequences are shown below (Table 3 and 4). Cloning and point mutations were confirmed using sequencing.

Table 3: Primers used for point mutations to insert AUG.

\begin{tabular}{l|r} 
Primer Type & Sequence \\
\hline Forward & 5' CATCCGTGCTGGCCCTTAGCGCCTCACCTAACAAC 3' \\
Reverse & 5' GTTGTTAGGTGAGGCGCTAAGGGCCAGCACGGATG 3'
\end{tabular}

Table 4: Primers used for point mutations to make end leader peptide with alanine and stop codon AUG.

\begin{tabular}{l|r} 
Primer Type & Sequence \\
\hline Forward & 5' CATCGCTGCTGGCGCATAGCGCCTCACCTAACAAC 3' \\
Reverse & 5' GTTGTTAGGTGAGGCGCTATGCGCCAGCAGCGATG 3'
\end{tabular}

The reaction to create the non-rescuable RNC was carried out at $37^{\circ} \mathrm{C}$ for 2 hours and the RNC was purified using Dynabeads ${ }^{\mathrm{TM}}$ His-Tag Isolation and Pulldown (Catalog Number 10104D) and by running on an InVision ${ }^{\mathrm{TM}}$ His-Tag In-Gel Stain (Catalog number LC6030) to visualize the produced peptide. For precise quantification of the nascent chain, $\mathrm{S}^{35}$ incorporation was optimized in S30 reactions. The length of the nascent chain was monitored using SDS-PAGE by running for 1 hour 
at $140 \mathrm{~V}$ and 3 hours at $60 \mathrm{~V}$ on a 16\% acrylamide gel in Tris-glycine buffer and imaging for 60 minutes. Non-rescuable-RNC production was attempted (Figure 20).

Suppressor tRNA Production The DNA construct for Suppressor tRNA for UAG was made using overlap extension PCR. An HDV ribozyme was programmed to cleave the suppressor tRNA at the CAA end to create a homogeneous 5 ' end. The cassette (Table 5) was made using overlap PCR using 20 cycles of a PCR reaction using $1 \mathrm{ul} \mathrm{Taq} 1 \mathrm{ul}$ DNTPs $5 \mathrm{ul}$ 10x buffer $2.5 \mathrm{ul}$ of both top and bottom DNA segments diluted in 38 ul water. The reaction was carried out over 20 cycles of 1 minute $94^{\circ} \mathrm{C}, 1$ minute $60^{\circ} \mathrm{C}$, and 30 seconds $72^{\circ} \mathrm{C}$. This DNA template was made into RNA using in vitro transcription (Described in Chapter 4). The construct for this tRNA was successfully made and sequenced.

Table 5: Sequence of the serine suppressor tRNA.

Sequence of the E.coli Ser-tRNA-2 amber suppressor supD60 gene 5' TAATACGACTCACTATAGGAGAGATGCCGGAGCGGCTGAACGGACCGGT CTCTAAAACCGGAGTAGGGGCAACTCTACCGGGGGTTCAAATCCСССTCTC TCCGCCA 3'

FtsY Production The bacterial Signal Recognition Particle (SRP) FtsY was created with a protocol similar to [12] except the cloning was done in the SLIC method [11] into vector pET11a. Cloning was confirmed via sequencing.

Isolation of Inner Membrane Vesicles Inner Membrane Vesicles (IMVs) are vesicles made of the plasma membrane. A method was developed to isolate IMVs in order to use as the membrane to which the SecM protein will be targeted. $\mathrm{K}-12 \mathrm{E}$. coli cells were grown to mid-log phase (about 0.8 ) shaking at $25^{\circ} \mathrm{C}$ in $\mathrm{M} 9$ 
minimal media [23] and then chilled to $4^{\circ} \mathrm{C}$ and harvested by a 30 minutes centrifugation at $3500 \mathrm{rpm}$. Pellets were stored in $200 \mathrm{mM}$ Tris pH 8, $1 \mathrm{mM} \mathrm{MgCl}_{2}$. Cells were suspended in 10 mM HEPES pH 7.6 and 1 mM EDTA and put through a french press twice at a pressure of $300 \mathrm{psi}$, with an addition of $1 \mathrm{mM}$ DTT between two passes. Lysate was centrifuged for 1,200 $\mathrm{g}$ for 20 minutes to remove intact cells. The supernatant was diluted to a protein concentration of $2.5 \mathrm{mg} / \mathrm{ml}$. Protein to lipid ratio is about $1: 1$ so the concentration of IMVs is about $5 \mathrm{mg} / \mathrm{ml}$ and then extruded with a $100 \mathrm{~nm}$ membrane an odd number of times.

\subsubsection{Optimizing AFM Imaging of Biological Samples in Liquid}

To image the components of co-translational translocation using AFM, an approach similar to previous work done to image SecYEG proteoliposomes [21]. The imaging optimization was initially performed on a purified ribosome, before adding in other components of the system such as FtsY or SecYEG (Figure 21). Prior to imaging, each sample is applied to a mica surface (V-1 grade, Structure Probe, Inc.) and imaged with Biolever mini tips (BL-AC40TS, Olympus) which have a spring constant of around $0.06 \mathrm{Newtons/meter.} \mathrm{Imaging} \mathrm{was} \mathrm{done} \mathrm{at} \mathrm{room} \mathrm{temperature} \mathrm{or}$ up to $30^{\circ} \mathrm{C}$ due to residual heat from the instrument. All images were taken on a Cypher commercial instrument from Asylum Research. Cypher operation and image analysis was done in Igor Pro. Image analysis included standard techniques such as filtering and flattening of an image to reduce noise and tilt. Heights of imaged ribosomes are found after the application of a floor mask that defines the background noise on the AFM images (Figure 22). 


\subsection{Results}

\subsubsection{Ribosome Nascent Chain Characterization}

The goal of the in vitro co-translational translation assay development is to recreate the in vitro system in a way that will allow for the quantification and observation of the influence of chaperone FtsY on the delivery of the ribosome to SecYEG during the making of an IMP to the membrane [15]. The intention is to develop the system and monitor the amount of membrane protein produced in the membrane in the presence and the absence of FtsY. This will allow for the quantification of FtsY's impact on the efficiency of membrane protein production.
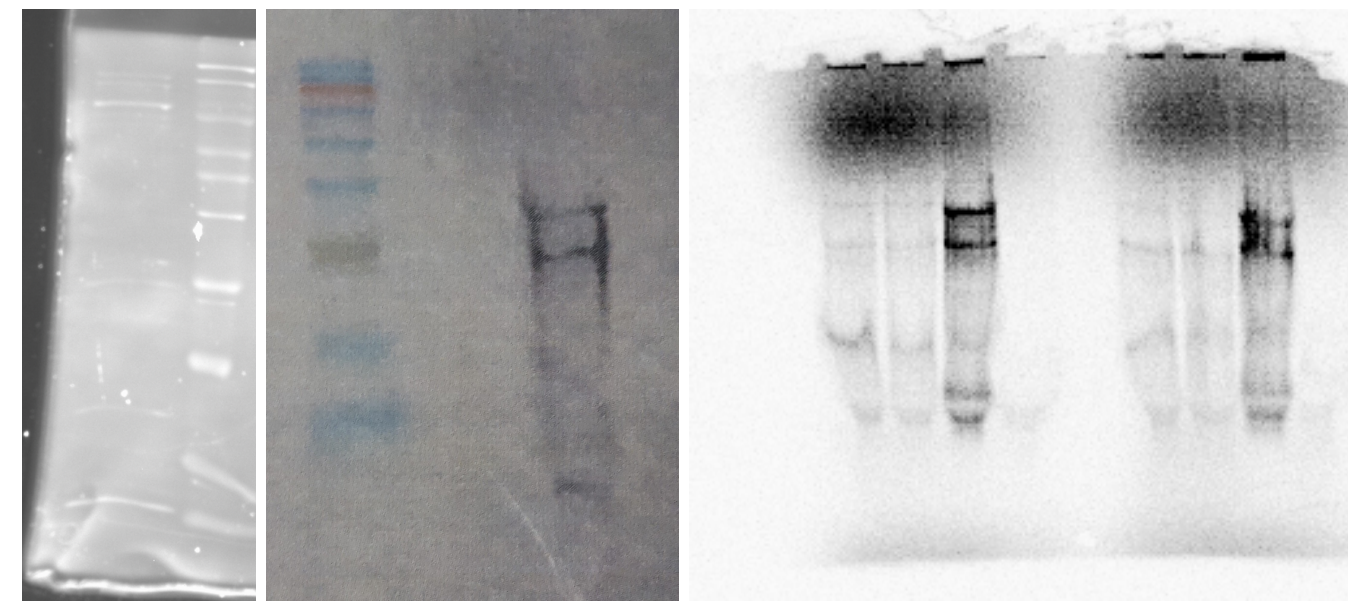

Figure 20: Product of In vitro Co-Translational Translation reactions on SDS-PAGE. Left: non-rescuable RNC with InVision ${ }^{\mathrm{TM}}$ His-Tag In-Gel Stain with ladder (ThermoFisher 26614) on right. Middle and left: S30 reaction with $S^{35}$ incorporation. Ladder is a gel picture overlayed by eye (NEB P7712).

Non-rescuable RNC may have been effectively made because a small band can be seen at the very bottom of the SDS-PAGE gel that was stained with InVision ${ }^{\mathrm{TM}}$ HisTag In-Gel Stain (Left, Figure 20). This staining observes his-tagged components in the reaction mixture, but the exact quantification or weight is difficult to decipher due to nonspecific staining. When the reaction was done with $S^{35}$, only bands for the positive control could be seen. Since the only difference is the DNA transcript, 
it is likely that the DNA transcripts are not properly cleaned or added to the reaction. Though the positive control bands at $39 \mathrm{kDa}$ for Cat fusion protein and 28 $\mathrm{kDa}$ for $\beta$ lac, prove that $\mathrm{S}^{35}$ tagging is a viable option for quantification of output IMPs from the in vitro co-translational translocation.
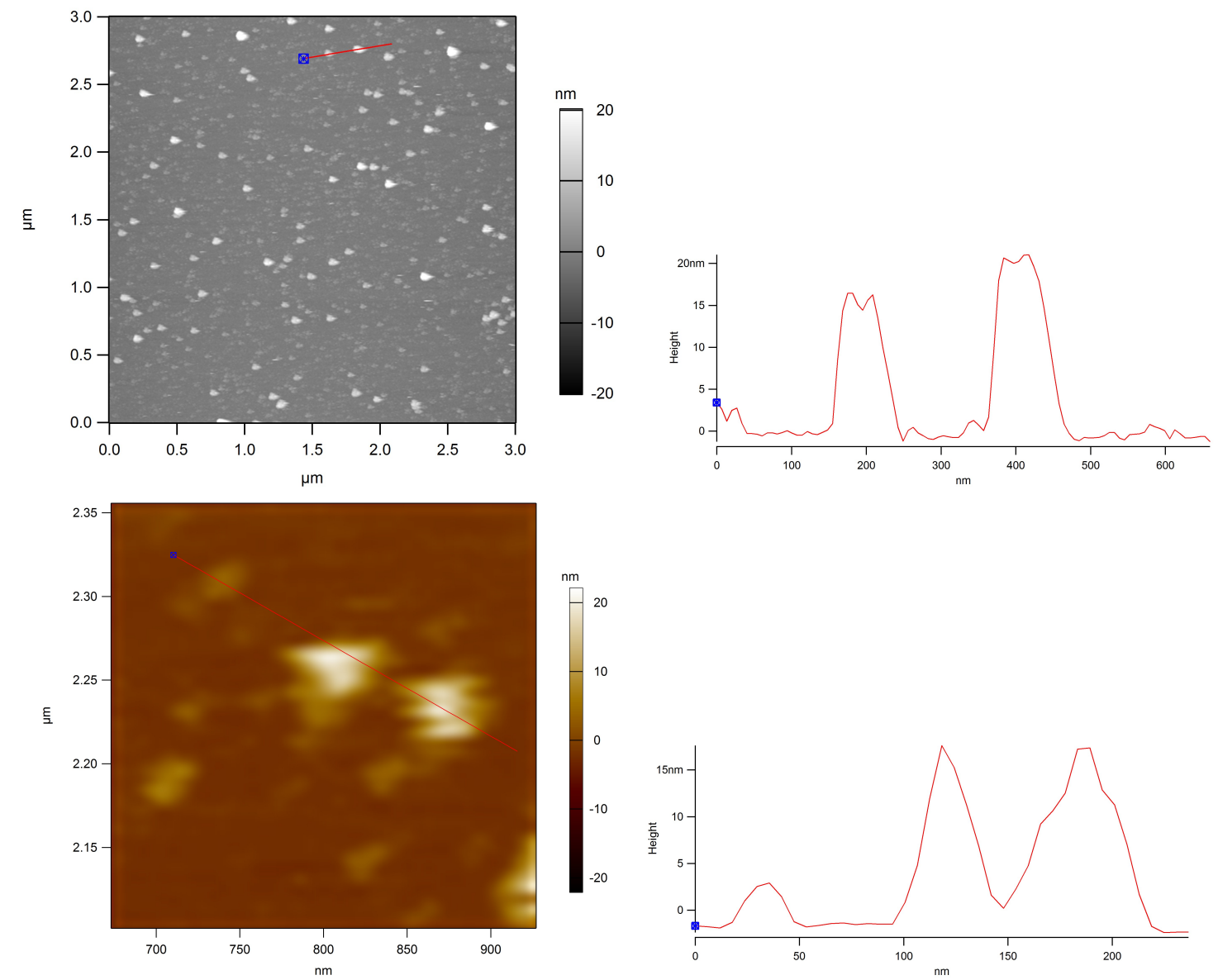

Figure 21: Topological report of Ribosomes on mica substrate. Top Left: Tapping mode AFM image of a $3 \mu \mathrm{m}$ by $3 \mu \mathrm{m}$ section of mica that has ribosomes deposited on it. Top Right: Height profile of the line drawn in top left image. Bottom Left: $200 \mathrm{~nm}$ by $200 \mathrm{~nm}$ tapping mode AFM image of ribosomes deposited on mica. Bottom Right: Height profile of the line drawn in bottom left image.

\subsubsection{The Ribosome on Mica Substrate: As Seen by AFM}

The intent of using AFM in tapping mode in liquid is to investigate the ribosome:SecYEG complex in the membrane. With the aim to find translocon:ribosome 
stoichiometry differences with and without FtsY delivery. SecYEG had already been visualized [15, 21]. Optimization of imaging the ribosome was the first step undertaken in this study. The purification method of the ribosomes had to be optimized to remove impurities that were in the ribosome samples when purified as described in [4]. The purification of the ribosomes was done with the method described in Section 2.1.3 and imaged (Figure 21).

The ribosomes imaged show an appropriate height of about $20 \mathrm{~nm}$ as the ribosome has an average diameter of about $20 \mathrm{~nm}$ (Figure 21] [5, 7, 9, 13, 14, 25]. Thus, it is reasonable that contact with the mica surface is not pulling apart the 70S into its subunits. The width of the particles in Figure 21 are about $100 \mathrm{~nm}$, which is about $80 \mathrm{~nm}$ larger than the ribosome's diameter. This increase is expected as the AFM tips used to image are in the shape of an upside-down cone such that a taller particle will come into contact with the wider edge before the atomically sharp tip does (Figure 19] [2]. The other possibility would be that two ribosomes are located next to each other. Due to this ambiguity, only the heights are an accurate report in this study. The tip-sample force during imaging ribosomes is approximately 100 $\mathrm{pN}$ or less [21]. It is necessary to understand the distortions that this could cause on the ribosomal particles. We assessed this by comparing the height of particles seen by a first pass of scanning (Figure 22 A) vs height of particles seen by the second pass of scanning (Figure 22 B). The height changes seen in the density histograms show a slight decrease in the average height of particles from the first scan to the second. The difference between these two histograms show there may be a height compression of about 2 to $3 \mathrm{~nm}$ on average from tip impact on biological particles. The average height is between 10 and $15 \mathrm{~nm}$; this indicates the even if some of the 70S stay associated, most ribosomes could be dissociating into $50 \mathrm{~S}$ and $30 \mathrm{~S}$ subunits (Figure 22). The smaller particles below $7 \mathrm{~nm}$ are either smaller parts 


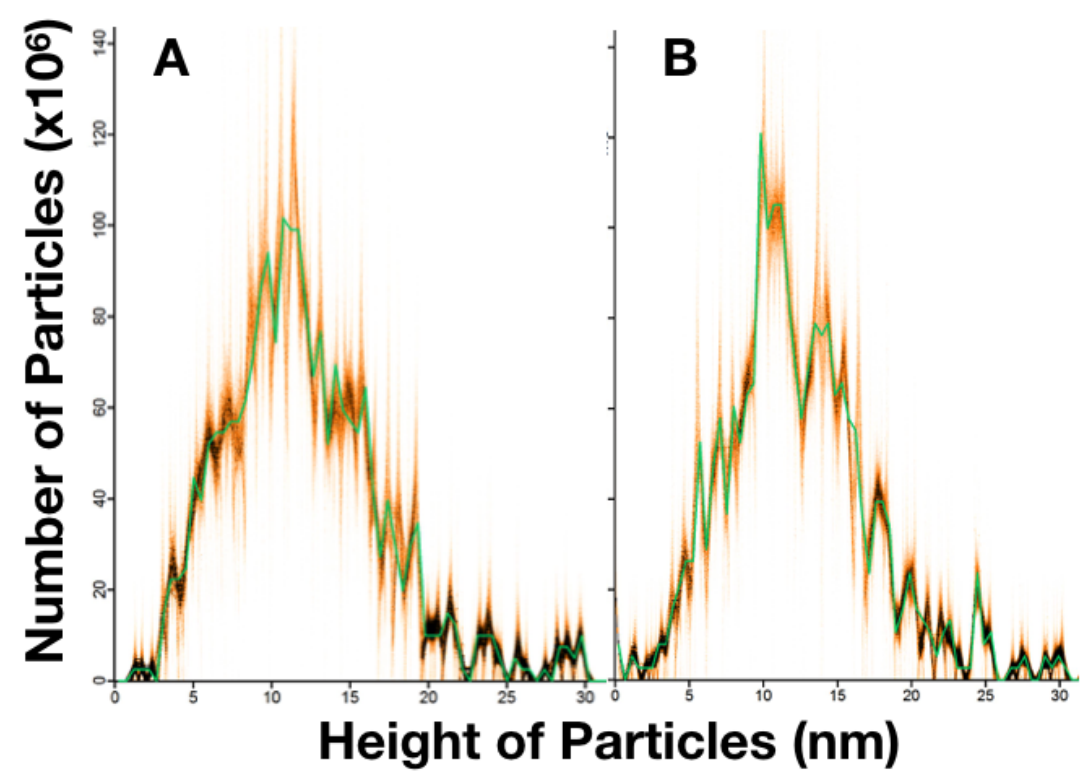

Figure 22: Height density histograms of imaged ribosomes show first pass heights (A) and second pass heights (B) of particles. Each density histogram plots density of height across all bin sizes

of the ribosome that have dissociated, such as protein uS1, or contamination.

\subsubsection{Ribosome Nascent Chain Non-specifically Binds to Lipid Bi- layer}

The aim of this project was to measure an active Ribosome:SecYEG complex using the AFM; the approach to do so includes many control experiments as mentioned previously with AFM imaging of the $70 \mathrm{~S}$ ribosome. Imaging an RNC was also undertaken to provide insights into possible dissociation of the $70 \mathrm{~S}$ into the $30 \mathrm{~S}$ and $50 \mathrm{~S}$ subunits and to detect possible nonspecific binding to a bilayer. Nonspecific binding is critical to quantify in AFM experiments of SecYEG binding [21]. Control experiments were performed to find possible nonspecific binding of RNCs to the membrane in order to accurately quantify the Ribosome:FtsY complex. The setup for this was to lay down a lipid bilayer onto the mica substrate and image the membrane (Figure 23, Top). Afterward, a non-rescuable RNC sample was added 

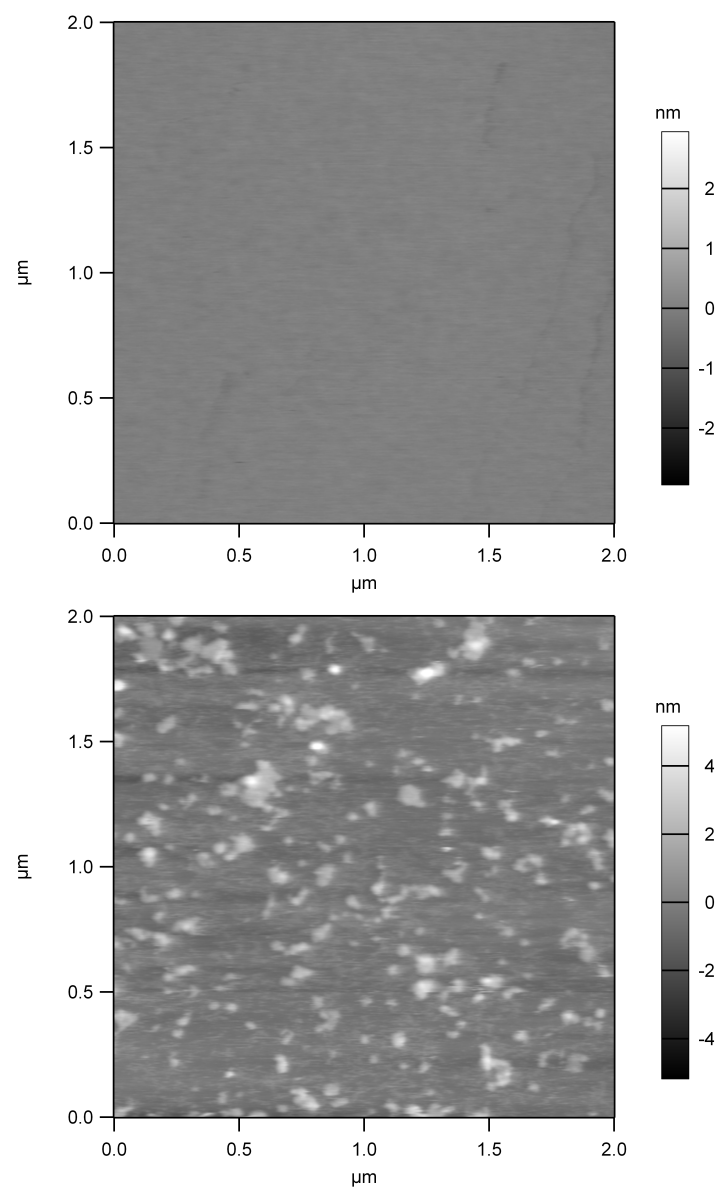

Figure 23: Top: AFM image of POPC on mica. Bottom: AFM image of RNC sample incubated over mica.

and incubated for 15 minutes at room temperature. After sample application, the RNC sample was washed 3 times for 5 minutes and then imaging continued (Figure 23, Bottom). The surface roughness from both images shown are equivalent to what is expected of a lipid surface, thus the contaminants seen in Figure 23 are bound to the POPC lipid bilayer. As the RNCs that were purified were from SecM stalled ribosomes as prepared with a nickel column [20], the contents are likely RNCs and possibly other things that non-specifically bind to a nickle column. These results show that nonspecific binding to the membrane can occur. This nonspecific binding is important to troubleshoot as nonspecific binding of the 
ribosome to the membrane will introduce uncertainty in AFM measurements of the ribosome:SecYEG complex.

\subsection{Discussion and Future Work}

Future work must be done with the in vitro co-translational translocation creation as well as AFM imaging. S30 reactions were accomplished as seen with the positive control, but the nascent chain was unable to be visualized with neither InVision ${ }^{\mathrm{TM}}$ His-Tag In-Gel Stain or with $S^{35}$ incorporation and radiography. Without precise quantification of the produced nascent chain, the assay would be insufficient to assess the activity of the co-translational translocation in vitro. Future work would be investigating and optimizing the DNA constructs and possibly redesign of the assay components, such as using a different stalling method for RNC creation.

Future work must be done to create suppressor tRNA. Specifically, in vitro translation will need to be done to produce the RNA oligo on large scale. The DNA of this suppressor tRNA was attempted to be cloned into Pet11a vector in order to amplify DNA for this purpose; this is the stage in which the work with suppressor tRNA stopped. Ultimately and theoretically the addition of this RNA oligo to the in vitro transcription/translation system would use the Serine tRNA synthetase to charge the serine amino acid on the tRNA CAA end and could restart translation in a rescuable RNC [19]. The efficiency of rescue would need to be quantified using a method of nascent peptide quantification, such as $S^{35}$ labeling.

Though it is expected that these RNCs will bind to SecYEG with high affinity [26], Figure 23] shows the RNC's binding to POPC. If RNCs are binding to POPC in a nonspecific manner, then we cannot assume that an RNC seen on the proteolipid membrane has SecYEG in it, and it cannot be inferred that the RNC is on top of and bound to a SecYEG channel. To investigate nonspecific binding of RNC to the 
membrane, the lipid content could be manipulated, but this would not be advisable as anionic phospholipids have been found to be enriched around the SecYEG translocon [6, 10] and the lipid content should not be manipulated. The main goal of the AFM aspect of this project was to image the RNC bound to SecYEG and see how this changes with FtsY. Though ribosomes were imaged, the goal of imaging the co-translation translocation machinery has yet to be accomplished (Figures 21 . Figure 22). Future work includes further purification of RNCs and optimizing the salts or procedure of sample application with the goal of minimizing nonspecific binding of RNCs on the membrane.

\section{References}

[1] Sandra Angelini, Sandra Deitermann, and Hans-Georg Koch. "FtsY, the bacterial signal-recognition particle receptor, interacts functionally and physically with the SecYEG translocon". In: EMBO Reports 6.5 (2005), pp. 476-481. ISSN: 1469-221X 1469-3178. DOI: $10.1038 / \mathrm{sj}$. embor . 7400385, URL: http: //www.ncbi.nlm.nih.gov/pmc/articles/PMC1299298/.

[2] A M Baró and R G Reifenberger. Atomic Force Microscopy in Liquid: Biological Applications. Apr. 2012. DOI: 10.1002/9783527649808.

[3] Peter Eaton and Paul West. Atomic force microscopy. 2010. DOI: 9780199570454. URL:https://global .oup.com/academic/product/atomic-force-microscopy9780199570454?cc=us\%7B \%5C\&\%7Dlang=en \%7B\%5C\&\%7D \%7B\%5C\#7D.

[4] Josefine Ederth et al. "A single-step method for purification of active Histagged ribosomes from a genetically engineered Escherichia coli". In: $\mathrm{Nu}$ cleic Acids Res 37.2 (Feb. 2009), e15-e15. ISSN: 0305-1048. DOI: 10.1093/nar/ gkn992. URL: http://www.ncbi.nlm.nih.gov/pmc/articles/PMC2632923// 
[5] Shu Feng, Yun Chen, and Yong Gui Gao. "Crystal Structure of 70S Ribosome with Both Cognate tRNAs in the E and P Sites Representing an Authentic Elongation Complex". In: PLoS ONE 8.3 (2013), pp. 1-11. ISSN: 19326203. DOI: 10.1371/journal.pone.0058829.

[6] Jens Frauenfeld et al. "Cryo-EM structure of the ribosome-SecYE complex in the membrane environment." eng. In: Nature structural \& molecular biology 18.5 (May 2011), pp. 614-621. ISSN: 1545-9985 (Electronic). DOI: 10 . $1038 /$ nsmb.2026.

[7] Haixiao Gao et al. "Study of the Structural Dynamics of the E. coli 70S Ribosome Using Real-Space Refinement Georgia Institute of Technology". In: Cell 113 (2003), pp. 789-801.

[8] Veit Goder and Martin Spiess. "Molecular mechanism of signal sequence orientation in the endoplasmic reticulum". In: The EMBO Journal 22.14 (July 2003), pp. 3645-3653. ISSN: 0261-4189. DOI: 10 . 1093/ emboj / cdg361. URL: https://doi.org/10.1093/emboj/cdg361.

[9] Patricia Julián et al. "Structure of ratcheted ribosomes with tRNAs in hybrid states". In: Proceedings of the National Academy of Sciences of the United States of America 105.44 (2008), pp. 16924-16927. ISSN: 00278424. DOI: 10.1073/pnas. 0809587105 .

[10] Wim van Klompenburg et al. "Anionic phospholipids are determinants of membrane protein topology". In: The EMBO Journal 16.14 (July 1997), pp. 42614266. ISSN: 0261-4189. DOI: 10.1093/emboj/16.14.4261. URL: https://doi. org/10.1093/emboj/16.14.4261

[11] Mamie Z Li and Stephen J Elledge. "SLIC: a method for sequence- and ligationindependent cloning." eng. In: Methods in molecular biology (Clifton, N.J.) 852 
(2012), pp. 51-59. ISSN: 1940-6029 (Electronic). DOI: $10.1007 / 978-1-61779-$ 564-0_5.

[12] Ottilie von Loeffelholz et al. "Ribosome-SRP-FtsY cotranslational targeting complex in the closed state." eng. In: Proceedings of the National Academy of Sciences of the United States of America 112.13 (Mar. 2015), pp. 3943-3948. ISSN: 1091-6490 (Electronic). DOI: 10.1073/pnas. 1424453112.

[13] Anna B Loveland and Andrei A Korostelev. "Structural dynamics of protein S1 on the 70S ribosome visualized by ensemble cryo-EM". In: Methods (San Diego, Calif.) 137 (Mar. 2018), pp. 55-66. ISSN: 1095-9130. DOI: 10.1016/ j. ymeth . 2017 . 12.004. URL: https : // www . ncbi .nlm . nih . gov/pubmed / 29247757\%20https://www.ncbi.nlm.nih.gov/pmc/PMC5866760/.

[14] Soneya Majumdar et al. "Disrupting domain-domain interactions is indispensable for EngA-ribosome interactions". In: Biochimica et Biophysica Acta Proteins and Proteomics 1865.3 (2017), pp. 289-303. ISSN: 18781454. DOI: 10. 1016/j . bbapap . 2016 .12.005. URL: http ://dx . doi .org/10.1016/j . bbapap.2016.12.005.

[15] Chunfeng Mao et al. "Stoichiometry of SecYEG in the active translocase of Escherichia coli varies with precursor species". eng. In: Proceedings of the National Academy of Sciences of the United States of America 110.29 (July 2013), pp. 11815-11820. ISSN: 1091-6490. DOI: 10 . 1073/ pnas . 1303289110. URL: https : //www .ncbi.nlm .nih.gov/pubmed/23818593\%20https : / / www . ncbi.nlm.nih.gov/pmc/articles/PMC3718118/

[16] D J Müller and A Engel. "The height of biomolecules measured with the atomic force microscope depends on electrostatic interactions". In: Biophysical Journal 73.3 (1997), pp. 1633-1644. ISSN: 0006-3495. DOI: https ://doi. 
org/10.1016/S0006-3495(97)78195-5. URL: http://wWw . sciencedirect. com/science/article/pii/S0006349597781955

[17] M Müller and G Blobel. “In vitro translocation of bacterial proteins across the plasma membrane of Escherichia coli". In: Proceedings of the National Academy of Sciences of the United States of America 81.23 (1984), pp. 7421-7425. ISSN: 0027-8424 1091-6490. URL: http: //www.ncbi.nlm.nih.gov/pmc/articles/ PMC392158/.

[18] Thomas R Noriega et al. "Signal Recognition Particle-ribosome Binding Is Sensitive to Nascent Chain Length". In: Journal of Biological Chemistry 289.28 (July 2014), pp. 19294-19305. URL: http : //www . jbc .org/content/289/28/ 19294.abstract.

[19] Erik A Rodriguez, Henry A Lester, and Dennis A Dougherty. "Improved amber and opal suppressor tRNAs for incorporation of unnatural amino acids in vivo. Part 1: Minimizing misacylation". In: RNA 13.10 (Oct. 2007), pp. 1703-1714. URL: http://rnajournal.cshlp.org/content/13/10/1703. abstract.

[20] Anna Rutkowska et al. "Large-scale purification of ribosome-nascent chain complexes for biochemical and structural studies". In: FEBS Letters 583.14 (2009), pp. 2407-2413. ISSN: 0014-5793. DOI: https : //doi .org/10 .1016/j . febslet . 2009.06.041. URL: http : / / wWw . sciencedirect . com/science / article/pii/S0014579309004992.

[21] Raghavendar Reddy Sanganna Gari et al. "Dynamic structure of the translocon SecYEG in membrane: direct single molecule observations". eng. In: The Journal of biological chemistry 288.23 (June 2013), pp. 16848-16854. ISSN: 1083351X. DOI: $10.1074 /$ jbc.M113.471870, URL: https : //www .ncbi.nlm .nih. 
gov/pubmed/23609442\%20https://www.ncbi.nlm.nih.gov/pmc/articles/ PMC3675617/,

[22] Karol Szkaradkiewicz et al. "RNA aptamers directed against release factor 1 from Thermus thermophilus." eng. In: FEBS letters 514.1 (Mar. 2002), pp. 9095. ISSN: 0014-5793 (Print). DOI: 10.1016/s0014-5793(02)02308-6.

[23] Törnkvist, M., Larsson, G. Enfors. “Protein release and foaming in Escherichia coli cultures grown in minimal medium". In: Bioprocess Engineering 15.231 (1996). DOI: https://doi.org/10.1007/BF02391583.

[24] Shuai Wang, Chien-I Yang, and Shu-Ou Shan. "SecA mediates cotranslational targeting and translocation of an inner membrane protein". eng. In: The Journal of cell biology 216.11 (Nov. 2017), pp. 3639-3653. ISSN: 1540-8140. DOI: 10 . 1083 / jcb . 201704036. URL: https : / / www . ncbi . nlm . nih . gov/ pubmed / 28928132\%20https : / / www . ncbi .nlm . nih . gov / pmc/articles / PMC5674894/.

[25] Paul C. Whitford and Karissa Y. Sanbonmatsu. "Simulating movement of tRNA through the ribosome during hybrid-state formation". In: Journal of Chemical Physics 139.12 (2013), pp. 1-9. ISSN: 00219606. DOI: 10 . 1063 / 1. 4817212 .

[26] Zht Cheng Wu et al. "Competitive Binding of the SecA ATPase and Ribosomes to the SecYEG Translocon". In: Journal of Biological Chemistry 287.11 (Mar. 2012), pp. 7885-7895. URL: http : //www . jbc . org/content/287/11/ 7885. abstract.

[27] Bin Zhang and Thomas F 3rd Miller. "Long-timescale dynamics and regulation of Sec-facilitated protein translocation." eng. In: Cell reports 2.4 (Oct. 
2012), pp. 927-937. ISSN: 2211-1247 (Electronic). DOI: 10 . 1016/ j . celrep . 2012.08 .039 .

[28] Jun Zhang et al. "Mechanisms of ribosome stalling by SecM at multiple elongation steps". In: eLife 4 (2015). Ed. by Sjors H W Scheres, e09684. ISSN: 2050084X. DOI: 10.7554/eLife.09684. URL: https://doi.org/10.7554/eLife. 09684

[29] Jie Zhou et al. "Crystal structures of 70S ribosomes bound to release factors RF1, RF2 and RF3". eng. In: Current opinion in structural biology 22.6 (Dec. 2012), pp. 733-742. ISSN: 1879-033X. DOI: 10.1016/j.sbi.2012.08.004. URL: https://www.ncbi.nlm.nih.gov/pubmed/22999888\%20https://www.ncbi. nlm.nih.gov/pmc/articles/PMC3982307/ 


\section{Perspectives in Expanding the Ribosomal Frontier}

This dissertation shows the ribosome to be a soft and flexible molecule and provides novel insights into ribosomal protein flexibility. Contributions of this work can be applied to any study of the ribosome; single-molecule, frozen, or otherwise.

This work shows that ribosomal proteins are highly flexible and extended into the solvent to a higher degree than is seen in cryo-EM and x-ray crystallography structures. The different ribosomal protein extensions should be accounted for during the analysis of FRET studies that monitor fluorophores bound to ribosomal proteins. Distance changes between two points reported in smFRET experiments are often analyzed as conformational changes of the rRNA structure and flexibility. For example, the ribosome has three main structural movements as observed by cryo-EM and x-ray crystallography. These movements are subunit ratcheting, $30 S$ head rotation, and L1 stalk flexing. Underlying each of these movements are rRNA hinges. Thus, as smFRET reports dynamics, it often will attribute the observed dynamics to the flexing structure of the rRNA. This study proposes that, along with the rRNA flexibility, the ribosomal proteins are highly flexible and play active roles in ribosome function. Further studies of the ribosomal protein extension and flexibility while tagged with a chromophore are necessary. SAXS shows that intrinsically disordered proteins collapse in solution when they are tagged with a chromophore [3]. It is reasonable that the attachment of a fluorophore may collapse a ribosomal protein because ribosomal proteins show characteristics of intrinsically disordered proteins in solution [1, 2]. The degree of collapse could be quantified using SAS of tagged ribosomes. If the ribosomal proteins are collapsing, the current interpretation that tagged ribosomal proteins report rRNA flexibility would be bolstered.

The extension of ribosomal proteins, as shown by SAS, can be applied in cryo-EM 
data analysis during modeling. In the stages of particle picking, 2D classification, and 3D modeling, the diameter of the particle is required as a manual input. The maximum particle distances obtained from SAS data can be used to define this parameter. It is the perspective of this work that cryo-EM and SAS are corroborative techniques. Cryo-EM can provide all-atom structures used to interpret SAS data, and SAS can provide a snapshot of the unfrozen molecule in solution to use in Cryo-EM analysis. In addition to this relationship, the combination of these techniques can be used to monitor structural changes that occur during the plunging process. Plunging is a sample processing technique for cryo-EM and the effects of plunging on the sample are currently unknown and highly debated. SAS is a companion technique that bridges the gap between single-molecule measurements of the ribosome in solution and global structure of the ribosome from cryo-EM and x-ray crystallography.

The unique insight offered by this work is that it compares two ribosomes while flexible in liquid with two microscopy techniques. The solution structure was found using SAS, which found the ribosomal proteins are flexible and can be highly extended. The less discussed highlight of this work is the imaging of the ribosome using tapping mode AFM in liquid. Similarities in these sample environments and in the ribosome purification technique allow for a nearly direct comparison of ribosome structure in solution using two "lenses". One lens observes the ribosomes properties as a polymer intercalated with liquid; the other observes the ribosomes properties as a spring on a surface (Figure 21). A more extensive set of AFM data would be necessary to obtain statistically sound measurements of the ribosome in solution on a mica substrate. The nature of the hydration layer of the ribosome could be investigated using this frontier. The ionic forces that an AFM tip is sensitive to can be used to describe the hydration layer in measurements. Taking 
AFM and SAS measurements of the ribosome in solution while changing the ions of the buffer can provide an understanding of ribosome structural and functional changes that occur in a changing environment. These studies could be applied to understanding how a ribosome functions in compartmentalized jobs within a cell.

\section{References}

[1] Saurav Mallik and Sudip Kundu. "A comparison of structural and evolutionary attributes of Escherichia coli and Thermus thermophilus small ribosomal subunits: signatures of thermal adaptation." eng. In: PloS one 8.8 (2013), e69898. ISSN: 1932-6203 (Electronic). DOI: 10.1371/journal.pone.0069898.

[2] Saurav Mallik and Sudip Kundu. "Molecular interactions within the halophilic, thermophilic, and mesophilic prokaryotic ribosomal complexes: clues to environmental adaptation." eng. In: Journal of biomolecular structure $\mathcal{E}$ dynamics 33.3 (2015), pp. 639-656. ISSN: 1538-0254 (Electronic). DOI: 10.1080/07391102. 2014.900457.

[3] Joshua A Riback et al. "Commonly used FRET fluorophores promote collapse of an otherwise disordered protein". In: Proceedings of the National Academy of Sciences 116.18 (2019), pp. 8889-8894. ISSN: 0027-8424. DOI:10.1073/pnas. 1813038116. URL: https://www.pnas.org/content/116/18/8889 


\section{Appendix}

\subsection{Supplementary Figures}

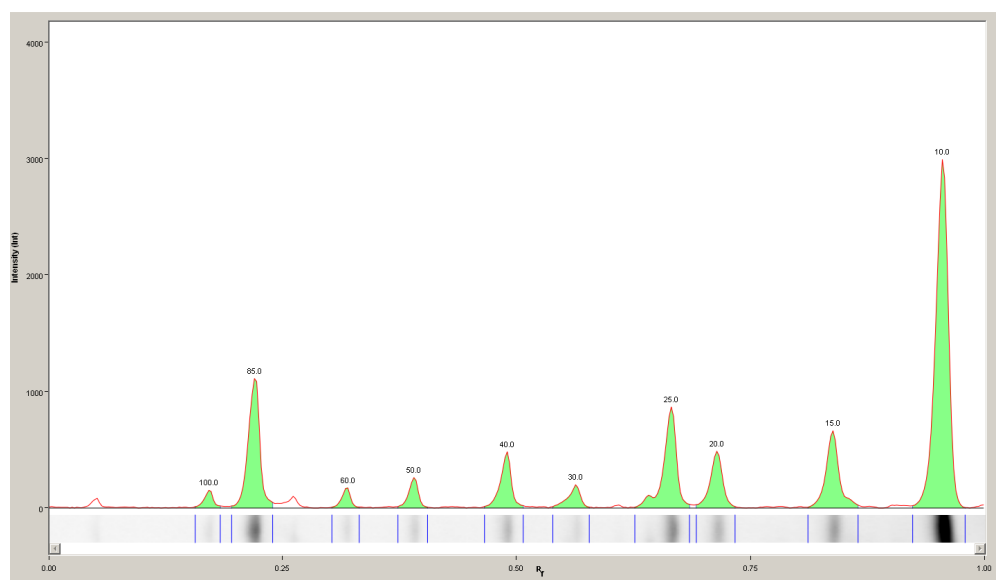

Figure S1: Profile of the protein standards used in SDS-PAGE gel in Chapter 2. Molecular weights are assigned.

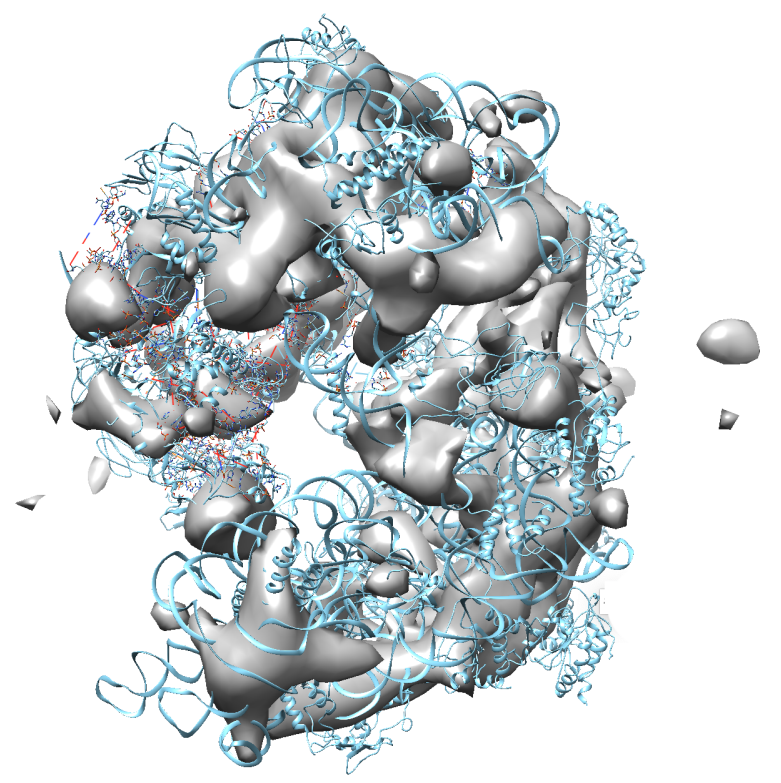

Figure S2: The 3D model of the 70S E. coli ribosome made using the software RELION in gray with PDB 3JCD of a $3.4 \AA$ Cryo-EM structure of the E. coli ribosome in blue. 


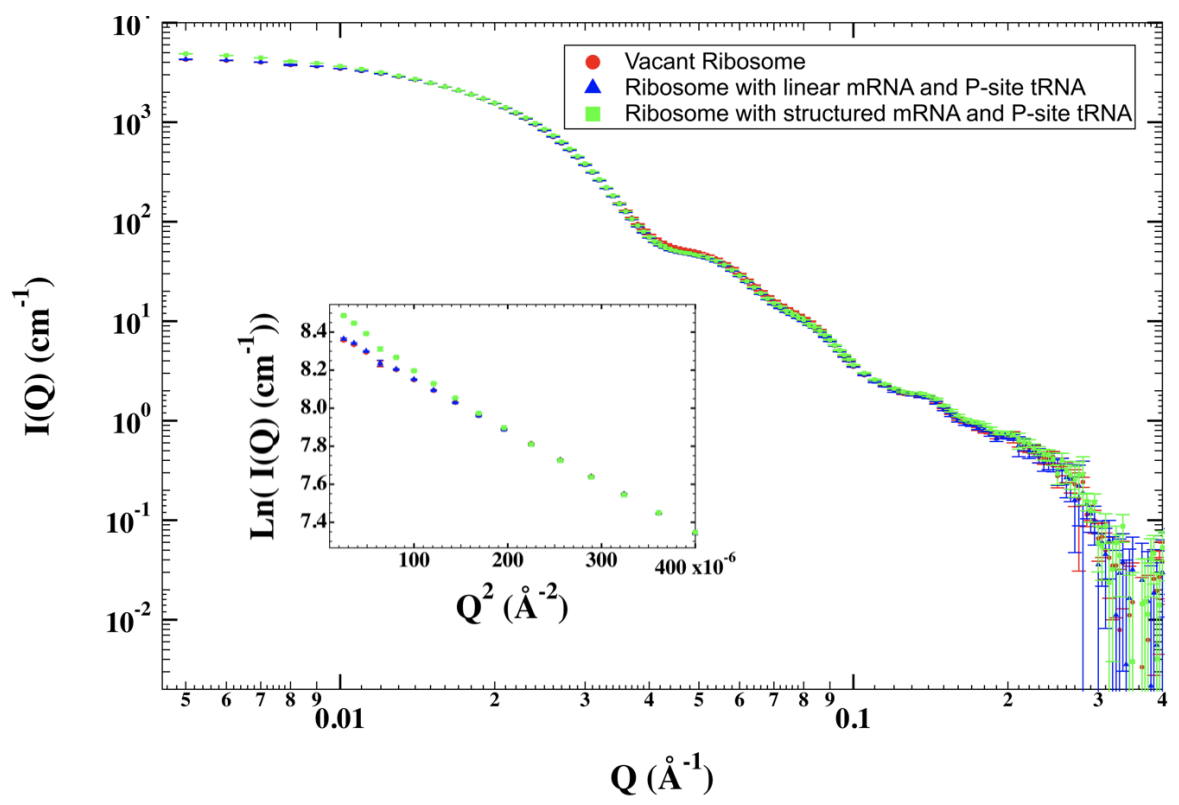

Figure S3: SAXS experimental curves overlayed for visual inspection. Inset is Guinier region of the curves.
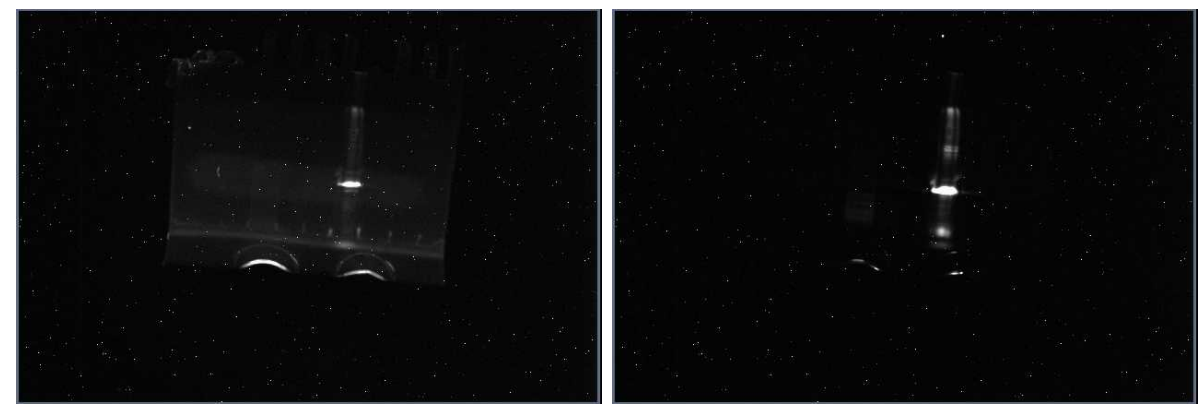

Figure S4: SDSPAGE gels of ribosomes. Left: Excitation at $550 \mathrm{~nm}$ to show Cy3. Right: Excitation at 649 to show Cy5. 
Table S1: Weights of 50 S large subunit proteins used to calculate histogram in Figure 20.

\begin{tabular}{|c|c|}
\hline protein & Uniprot weight (Da) \\
\hline I1 & 24,730 \\
\hline 12 & 29,860 \\
\hline 13 & 22,244 \\
\hline 14 & 22,087 \\
\hline 15 & 20,302 \\
\hline 16 & 18,904 \\
\hline 17 & 12,295 \\
\hline 17 & 12,295 \\
\hline \multicolumn{2}{|l|}{18} \\
\hline 19 & 15,769 \\
\hline$\lfloor 10$ & 17,712 \\
\hline I11 & 14,875 \\
\hline$\lfloor 12$ & 12,295 \\
\hline I12 & 12,295 \\
\hline$\lfloor 13$ & 16,019 \\
\hline I14 & 13,541 \\
\hline L15 & 14,980 \\
\hline L16 & 15,281 \\
\hline \17 & 14,365 \\
\hline \18 & 12,770 \\
\hline L19 & 13,133 \\
\hline 120 & 13,497 \\
\hline 121 & 11,564 \\
\hline 122 & 12,226 \\
\hline 123 & 11,199 \\
\hline 124 & 11,316 \\
\hline 125 & 10,693 \\
\hline \multicolumn{2}{|l|}{126} \\
\hline 127 & 9,124 \\
\hline 128 & 9,006 \\
\hline 129 & 7,273 \\
\hline 130 & 6,542 \\
\hline 131 & 7,871 \\
\hline L32 & 6,446 \\
\hline L33 & 6,372 \\
\hline L34 & 5,380 \\
\hline L35 & 7,289 \\
\hline L36 & 4,364 \\
\hline
\end{tabular}


Table S2: Weights of $30 \mathrm{~S}$ small subunit proteins used to calculate histogram in Figure 7 .

\begin{tabular}{|l|r|}
\hline protein & Uniprot weight (Da) \\
\hline$s 2$ & 26,744 \\
\hline$s 3$ & 25,983 \\
\hline$s 4$ & 23,469 \\
\hline$s 5$ & 17,603 \\
\hline$s 6$ & 15,703 \\
\hline$s 8$ & 20,019 \\
\hline$s 9$ & 14,127 \\
\hline$s 10$ & 14,856 \\
\hline$s 11$ & 11,736 \\
\hline$s 12$ & 13,845 \\
\hline$s 13$ & 13,737 \\
\hline$s 14$ & 13,099 \\
\hline$s 15$ & 11,580 \\
\hline$s 16$ & 10,269 \\
\hline$s 17$ & 9,191 \\
\hline$s 18$ & 9,704 \\
\hline$s 19$ & 8,986 \\
\hline$s 20$ & 10,430 \\
\hline$s 21$ & 9,684 \\
\hline
\end{tabular}

Table S3: Oligo sequences that were bought from Integrated DNA Technologies and bound to ribosomes as mRNA templates in Chapter 3. To detail where these oligos sit in the ribosome, the P-site AUG start codon is red and DnaX hairpin is green.

\begin{tabular}{|l|l|}
\hline mRNA Sequences \\
\hline DnaX & CUU AAU AAG GAA AUA AAA AUG UUU AGU GAA CCG GCA GCC GCU ACC CGC GCG CGG CCG GU \\
\hline$m 291$ & AUU AAA AAG GAA AUA AAA AUG UUU GUA UAC AAA UCU ACU GCU GAA \\
\hline
\end{tabular}


Table S4: Extended Guinier analysis of ribosomes bound to structured mRNA in $100 \% \mathrm{D}_{2} \mathrm{O}$ buffer.

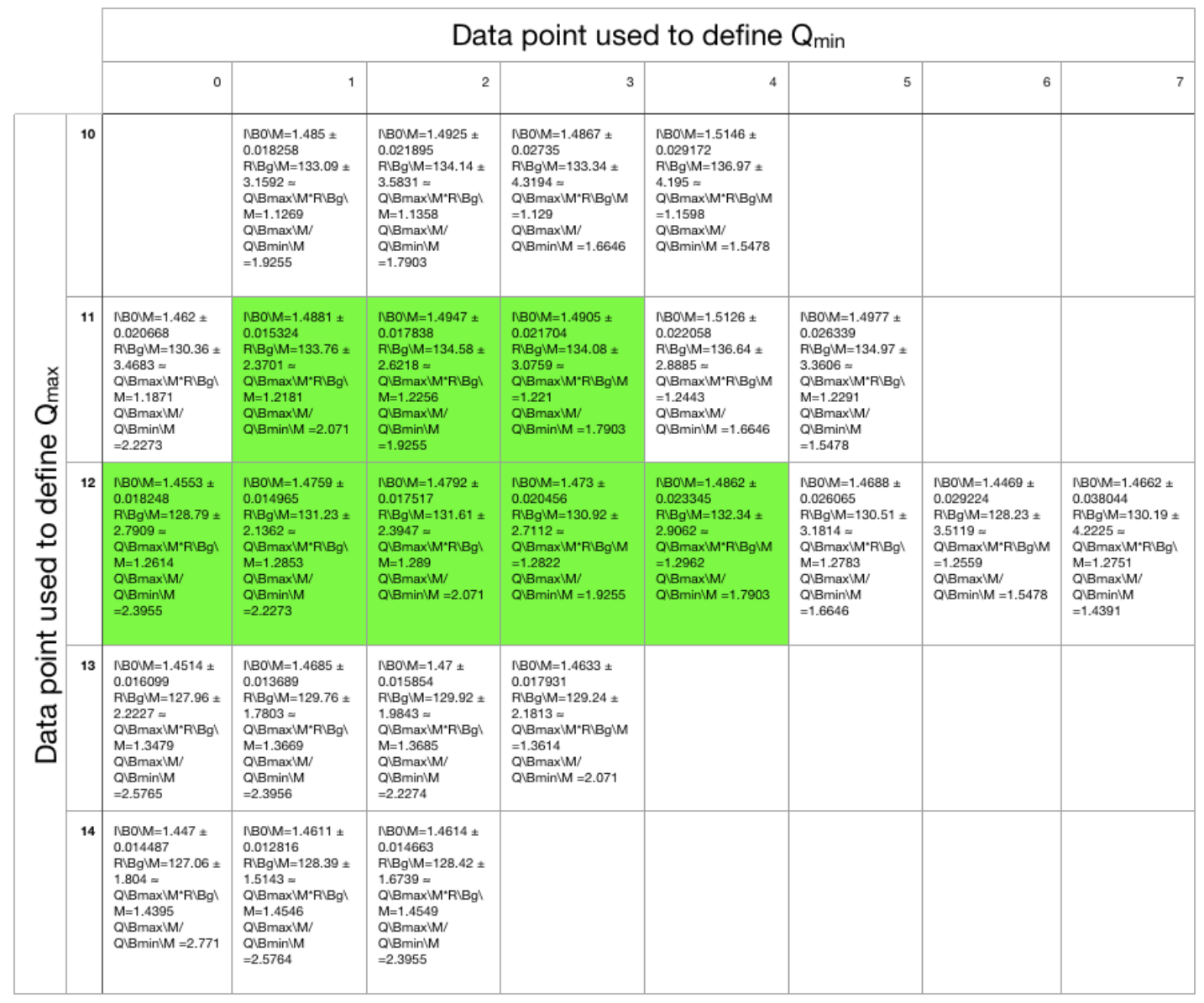

Table S5: Sequence of cloned m291 DNA.

m291 DNA sequence

5'TAATACGACTCACTATAGGTAAAGTGTCATAGCACCAACTGTTAATTAAATTAAATTAA AAAGGAAATAAAAATGTTTGTATACAAATCTACTGCTGAACTCGCTGCACAAATGGCTA AACTGAATGGCAATAAAGGTTTTTCTTCTGAAGATAAAGGATATCGGATCCTCT3', 
Table S6: Maximum dimension, $\mathrm{D}_{\max }$, calculated from the all atom models.

\begin{tabular}{|c|cc|ccc|c|}
\hline \multirow{2}{*}{$\begin{array}{c}\text { PDB } \\
\text { Models }\end{array}$} & \multicolumn{5}{|c|}{ Maximum Diameter, Dmax $(\AA)$} \\
\cline { 2 - 7 } & SAXS & \multicolumn{4}{c|}{ SANS } & VMD \\
\cline { 2 - 7 } & Vacant & Filled & $\begin{array}{c}\text { rRNA } \\
\text { (42\% D2O } \\
\text { buffer) }\end{array}$ & $\begin{array}{c}\text { Vacant } \\
\text { (100\% D2O } \\
\text { buffer) }\end{array}$ & $\begin{array}{c}\text { Filled } \\
(100 \% \text { D2O } \\
\text { buffer })\end{array}$ & Filled \\
\hline 4v7a & 282.3 & 282.3 & 264.8 & 282.3 & 282.3 & 264.0 \\
4v9d 1 & 277.9 & 277.9 & 260.7 & 277.9 & 277.9 & 272.0 \\
4v9d 2 & 276.9 & 276.9 & 261.1 & 276.9 & 276.9 & 264.0 \\
6bu8 & 278.7 & 278.4 & 265.4 & 278.7 & 278.4 & 270.0 \\
5afi & 272.2 & 272.6 & 263.9 & 272.2 & 272.6 & 252.0 \\
4woi 1 & 278.7 & 278.8 & 262.0 & 278.7 & 278.8 & 275.0 \\
4woi 2 & 280.7 & 280.8 & 263.4 & 280.7 & 280.8 & 264.0 \\
3JCD & 276.0 & 277.9 & 268.0 & 276.0 & 277.9 & 258.0 \\
4v7b & 274.8 & 274.7 & 264.7 & 274.8 & 274.7 & 265.0 \\
4v7d & 269.9 & 274.6 & 264.7 & 269.9 & 274.6 & 264.0 \\
4v7c & 267.2 & 271.7 & 261.0 & 267.2 & 271.7 & 264.0 \\
6h4n & 275.5 & 275.3 & 264.0 & 275.5 & 275.3 & 265.0 \\
\hline sum & 275.9 & 276.8 & 263.6 & 275.9 & 276.8 & 264.8 \\
\hline stdev & 4.4 & 3.2 & 2.2 & 4.4 & 3.2 & 6.0 \\
\hline
\end{tabular}


Table S7: Radius of gyration, $R_{g}$, calculated from the all atom models.

\begin{tabular}{|c|cc|ccc|c|}
\hline \multirow{2}{*}{$\begin{array}{c}\text { PDB } \\
\text { Models }\end{array}$} & \multicolumn{6}{|c|}{ Radius of gyration, Rg $(\AA)$} \\
\cline { 2 - 7 } & SAXS & \multicolumn{4}{c|}{ SANS } & VMD \\
\cline { 2 - 7 } & Vacant & Filled & $\begin{array}{c}\text { rRNA } \\
\text { (42\% D2O } \\
\text { buffer) }\end{array}$ & $\begin{array}{c}\text { Vacant } \\
(100 \% \text { D2O } \\
\text { buffer })\end{array}$ & $\begin{array}{c}\text { Filled } \\
(100 \% \text { D2O } \\
\text { buffer })\end{array}$ & Filled \\
\hline 4v7a & 85.8 & 85.4 & 82.3 & 89.2 & 88.9 & 85.9 \\
4v9d 1 & 83.1 & 83.2 & 80.5 & 86.0 & 86.0 & 83.4 \\
4v9d 2 & 83.8 & 83.9 & 81.1 & 87.1 & 87.1 & 84.3 \\
6bu8 & 84.9 & 84.0 & 82.9 & 88.3 & 87.6 & 84.5 \\
5afi & 84.2 & 83.4 & 80.5 & 86.7 & 86.3 & 83.9 \\
4woi 1 & 84.2 & 84.0 & 81.1 & 87.4 & 87.2 & 84.3 \\
4woi 2 & 84.7 & 84.3 & 81.4 & 87.7 & 87.5 & 84.6 \\
3JCD & 85.5 & 85.2 & 82.3 & 88.6 & 88.3 & 85.6 \\
4v7b & 85.2 & 84.4 & 81.2 & 88.4 & 88.1 & 84.9 \\
4v7d & 84.7 & 83.9 & 81.2 & 87.6 & 87.1 & 84.3 \\
4v7c & 84.4 & 83.8 & 80.8 & 87.5 & 87.2 & 84.2 \\
6h4n & 84.9 & 84.7 & 80.3 & 87.3 & 87.7 & 85.0 \\
\hline SUM & 84.6 & 84.2 & 81.3 & 87.7 & 87.4 & 84.6 \\
\hline STDEV & 0.7 & 0.7 & 0.8 & 0.9 & 0.8 & 0.7 \\
\hline
\end{tabular}


Table S8: smFRET histogram fitting parameters.

\begin{tabular}{|c|c|c|c|c|c|c|c|}
\hline \multicolumn{4}{|l|}{ M291 } & \multicolumn{4}{|l|}{ BS2 } \\
\hline \multicolumn{4}{|c|}{$\begin{array}{l}\text { Chi square: } 0.56026 \\
\text { Total fitted points: } 20 \\
\text { Multi-peak fit version } 2.22 \\
\text { Total Peak Area }=9.3088+/-0.42864\end{array}$} & \multicolumn{4}{|c|}{$\begin{array}{l}\text { Chi square: } 6.1856 \\
\text { Total fitted points: } 20 \\
\text { Multi-peak fit version } 2.22 \\
\text { Total Peak Area }=7.0545+/-1.7502 \\
\end{array}$} \\
\hline \multicolumn{4}{|c|}{ Baseline Type: Constant } & \multicolumn{4}{|c|}{ Baseline Type: Constant } \\
\hline & $\mathrm{y} 0=$ & $0.17874+/-$ & 0.080865 & & $\mathrm{y} 0=$ & $0.28556+/-$ & 0.31128 \\
\hline \multirow[t]{8}{*}{ Peak 0} & \multicolumn{3}{|c|}{ Type: Gauss } & \multicolumn{4}{|c|}{ Peak 0 Type: Gauss } \\
\hline & Location $=$ & $0.4205+/-$ & 0.003622 & & Location $=$ & $0.45712+/-$ & 0.020486 \\
\hline & Height $=$ & $28.34+/-$ & 0.86105 & & Height $=$ & $23.253+/-$ & 2.5435 \\
\hline & Area $=$ & $6.2747+/-$ & 0.30141 & & Area $=$ & $6.3673+/-$ & 1.2427 \\
\hline & \multicolumn{3}{|c|}{ Fit function parameters } & \multicolumn{4}{|c|}{ Fit function parameters } \\
\hline & Location $=$ & $0.4205+/-$ & 0.003622 & & Location $=$ & $0.45712+/-$ & 0.020486 \\
\hline & Width $=$ & $0.12492+/-$ & 0.0024722 & & Width $=$ & $0.15449+/-$ & 0.014405 \\
\hline & Height $=$ & $28.34+/-$ & 0.86105 & & Height $=$ & $23.253+/-$ & 2.5435 \\
\hline \multirow[t]{8}{*}{ Peak 1} & \multicolumn{3}{|c|}{ Type: Gauss } & \multicolumn{4}{|c|}{ Peak 1 Type: Gauss } \\
\hline & Location $=$ & $0.60432+/-$ & 0.0094071 & & Location $=$ & $0.65817+/-$ & 0.14808 \\
\hline & Height $=$ & $12.6+/-$ & 0.68577 & & Height $=$ & $2.7373+/-$ & 3.2482 \\
\hline & Area $=$ & $3.0341+/-$ & 0.30477 & & Area $=$ & $0.68728+/-$ & 1.2325 \\
\hline & $\begin{array}{l}\mathrm{FWHM}= \\
\text { Fit function }\end{array}$ & $\begin{array}{l}0.22623+/- \\
\text { parameters }\end{array}$ & 0.011402 & & $\begin{array}{l}\text { FWHM = } \\
\text { Fit function }\end{array}$ & $\begin{array}{l}0.23588+/- \\
\text { parameters }\end{array}$ & 0.16027 \\
\hline & Location $=$ & $0.60432+/-$ & 0.0094071 & & Location $=$ & $0.65817+/-$ & 0.14808 \\
\hline & Width $=$ & $0.13586+/-$ & 0.0068476 & & Width $=$ & $0.14166+/-$ & 0.096255 \\
\hline & Height $=$ & $12.6+/-$ & 0.68577 & & Height $=$ & $2.7373+/-$ & 3.2482 \\
\hline
\end{tabular}

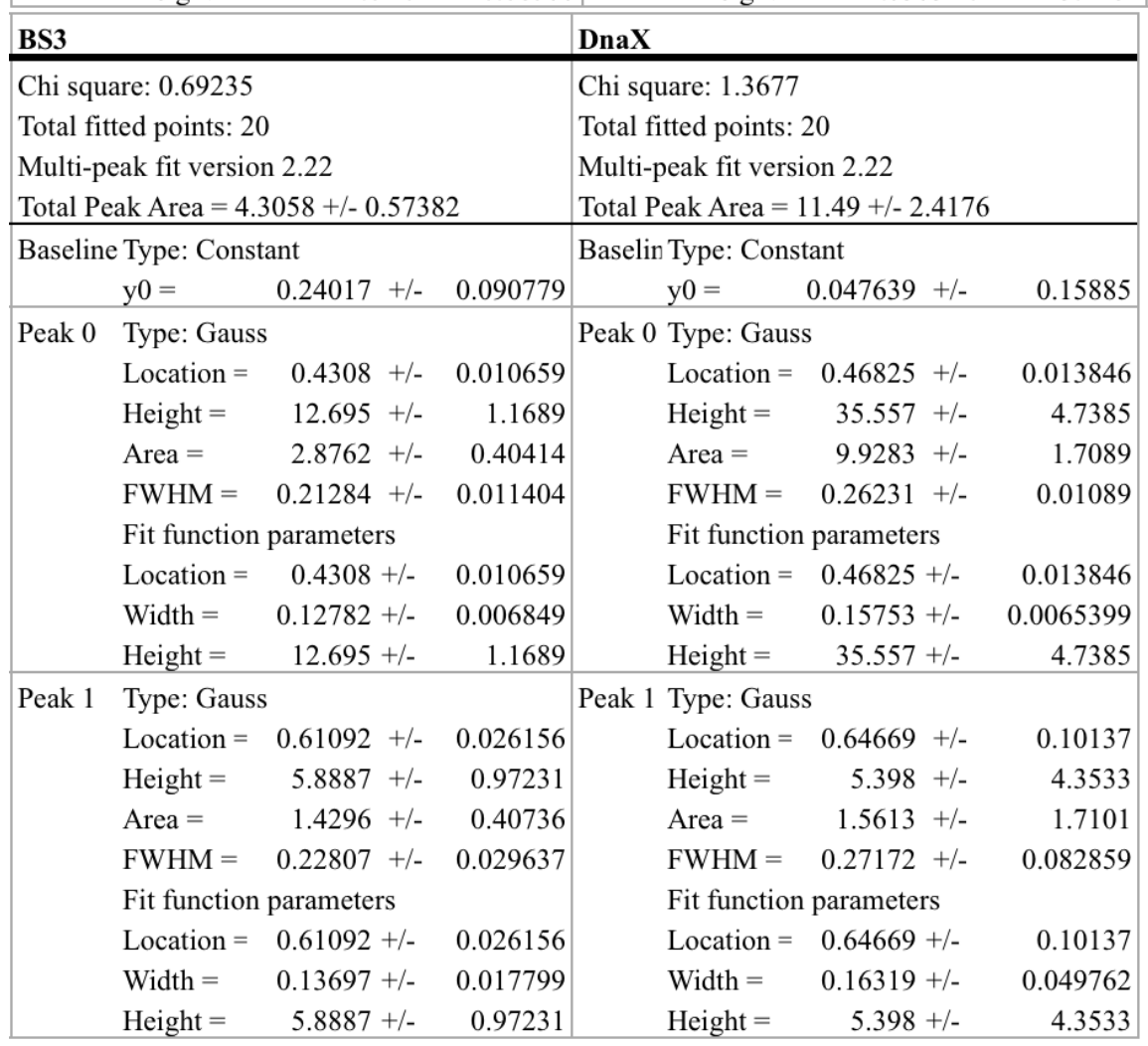




\section{VITA}

Emily Doris Armbruster was born on May 21st, 1991 and raised in Ann Arbor, Michigan. She obtained a Bachelor of Science degree in Biology and Chemistry from Hope College, located in Holland, Michigan in May of 2013. Emily received her Doctoral degree in Biochemistry from the University of Missouri, located in Columbia, Missouri in December of 2019. 